Florida International University FIU Digital Commons

6-29-2018

\title{
Novel Role of Pseudomonas Aeruginosa LptD Operon
}

Sundar Pandey

spand005@fiu.edu

DOI: $10.25148 /$ etd.FIDC006902

Follow this and additional works at: https://digitalcommons.fiu.edu/etd

Part of the Bacteriology Commons, Biology Commons, and the Pathogenic Microbiology Commons

\section{Recommended Citation}

Pandey, Sundar, "Novel Role of Pseudomonas Aeruginosa LptD Operon" (2018). FIU Electronic Theses and Dissertations. 3734. https://digitalcommons.fiu.edu/etd/3734

This work is brought to you for free and open access by the University Graduate School at FIU Digital Commons. It has been accepted for inclusion in FIU Electronic Theses and Dissertations by an authorized administrator of FIU Digital Commons. For more information, please contact dcc@fiu.edu. 


\section{FLORIDA INTERNATIONAL UNIVERSITY}

Miami, Florida

NOVEL ROLE OF PSEUDOMONAS AERUGINOSA LPTD OPERON

A dissertation submitted in partial fulfillment of

the requirements for the degree of

DOCTOR OF PHILOSOPHY

in

BIOLOGY

by

Sundar Pandey 
To: Dean Michael R. Heithaus

College of Arts, Sciences and Education

This dissertation, written by Sundar Pandey, and entitled Novel Role of Pseudomonas aeruginosa lptD Operon, having been approved in respect to style and intellectual content, is referred to you for judgment.

We have read this dissertation and recommend that it be approved.

$\begin{array}{r}\hline \text { Fenfei Leng } \\ \hline \text { Fernando Noriega } \\ \hline \text { Joanna Goldberg } \\ \hline \text { John Makemson } \\ \hline \text { Kalai Mathee, Major Professor }\end{array}$

Date of Defense: June 29, 2018

The dissertation of Sundar Pandey is approved.

Dean Michael R. Heithaus

College of Arts, Sciences and Education

Andrés G. Gil

Vice President for Research and Economic Development and Dean of the University Graduate School

Florida International University, 2018 


\section{ACKNOWLEDGMENTS}

I would like to thank my Ph.D. advisor Dr. Kalai Mathee for accepting me in her lab. Likewise, I would also like to thank my committee members: Dr. Fenfei Leng, Dr. John Makemson, Dr. Fernando Noriega, and Dr. Joanna Goldberg for their guidance throughout the years. Dr. Mathee's lab has been a place to meet with wonderful people to exchange scientific ideas.

I am grateful to the financial support provided by the university graduate school (presidential fellowship), department of biological sciences (TAship), the FIU Biomedical Research Initiative student summer research award.

Finally, I would like to thank my family and friends for being supportive and patient. 


\section{ABSTRACT OF THE DISSERTATION \\ NOVEL ROLE OF PSEUDOMONAS AERUGINOSA LPTD OPERON}

by

Sundar Pandey

Florida International University, 2018

Miami, Florida

Professor Kalai Mathee, Major Professor

Pseudomonas aeruginosa is an opportunistic pathogen that infects cystic fibrosis (CF) patients contributing to their high morbidity and mortality. P. aeruginosa undergoes a phenotypic conversion in the CF lung, from nonmucoid to mucoid, by constitutively producing a polysaccharide called alginate. These mucoid strains often revert to nonmucoid in vitro due to second-site suppressor mutations. We hypothesized that mapping these mutations would lead to the identification of novel genes involved in alginate production. In a previous study, a mucoid strain, PDO300 (PAOmисA22), was used to isolate suppressors of alginate phenotype (sap). One of the uncharacterized nonmucoid revertants, sap27, is the subject of this study. The mucoid phenotype in sap27 was restored by pMO012217 from a minimal tiling path cosmid library. The cosmid pMO012217 harbors $18 P$. aeruginosa open reading frames (ORF). The cosmid was mutagenized with a transposon to map the contributing gene. It was mapped to lptD (PA0595) encoding lipopolysaccharide transport protein. E. coli LptD transports lipopolysaccharide to the outer leaflet of the outer membrane. The $\mathrm{Alg}^{+}$phenotype was restored upon complementation with $P$. aeruginosa $l p t D$ alone, suggesting that sap27 likely harbor a chromosomal mutation in $l p t D$. Sequencing analysis of sap27 showed the 
presence of a mutation not in $\operatorname{lptD}$ but in $\operatorname{alg} O$, which encodes a periplasmic protease protein. This suggests $\mathrm{LptD}$ is able to bypass an $\operatorname{alg} O$ mutation by positively regulating alginate production. The $\operatorname{lpt} D$ is a part of a three-gene operon $l p t D-s u r A-p d x A$. SurA is an essential protein for survival in starvation and a major chaperone protein for all outer membrane proteins and $\mathrm{PdxA}$ is a NAD-dependent dehydrogenase and is involved in the vitamin $\mathrm{B}_{6}$ biosynthetic pathway. Pyridoxal 5'-phosphate (PLP) is the active form of vitamin $\mathrm{B}_{6}$. P. aeruginosa grown in a media supplemented with PLP increased production of pyocyanin, a virulence factor. The PLP and aromatic amino acids are synthesized from a common precursor chorismic acid. We demonstrated an increase in pyocyanin production when the bacteria were cultured supplemented by the aromatic amino acids phenylalanine. We concluded that the lptD operon plays a role in the $P$. aeruginosa virulence by regulating alginate and pyocyanin production. 


\section{TABLE OF CONTENTS}

\section{CHAPTER}

\section{PAGE}

CHAPTER 1

Posttranslational regulation of antisigma factors of RpoE: a comparison between the Escherichia coli and Pseudomonas aeruginosa .......................................................

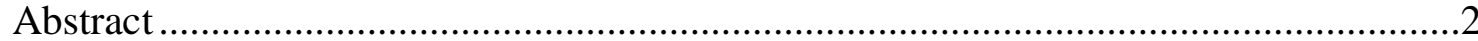

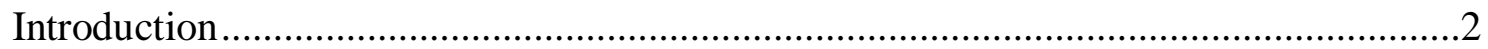

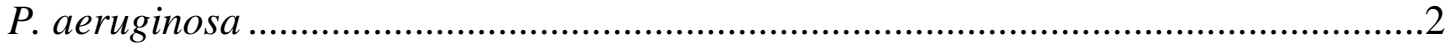

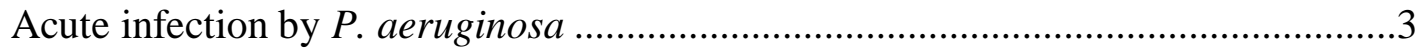

i. Acute pneumonia in hospitalized and in particular mechanically

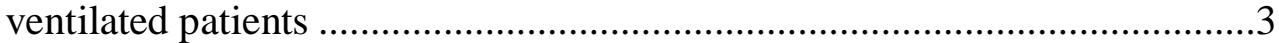

ii. Sepsis in patients with extensive burns .................................................4

iii. Corneal infection in individuals wearing contact lenses..............................4

iv. Urinary tract infection in patients with indwelling foley catheters................5

v. Bacteremia and sepsis in immunocompromised patient, particularly neutropenic patients receiving cytotoxic therapies ......................................

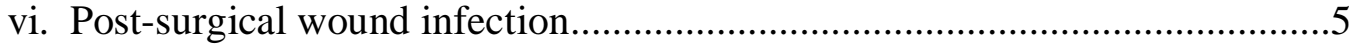

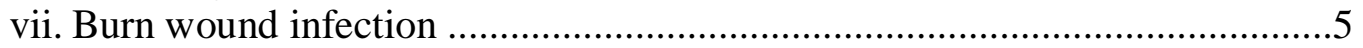

Chronic infection by $P$. aeruginosa ................................................................6

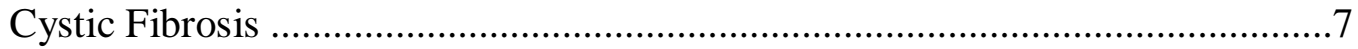

Microbial communities in $\mathrm{CF}$ airways ................................................. 11

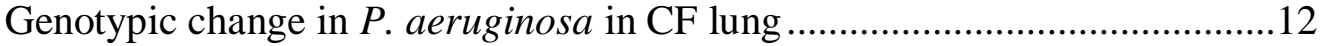



Biosynthesis of alginate .......................................................................... 15

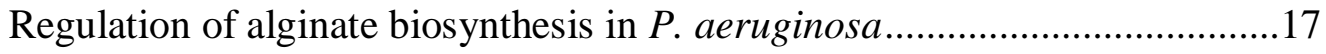

Regulated intramembrane proteolysis cascade .......................................20

Extracytoplasmic stress sigma factor $\sigma^{22}$ : AlgT/U and RpoE ................21

E. coli RseA and P. aeruginosa MucA-anti sigma factor.........................22

E. coli RseB and $P$. aeruginosa MucB-Anti-anti

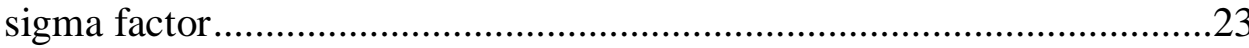

E. coli RseC and P. aeruginosa MucC ................................................26

E. coli DegP and P. aeruginosa MucD ................................................26

E. coli DegS and P. aeruginosa AlgW ...............................................27

E. coli ResP and P. aeruginosa MucP ..............................................28



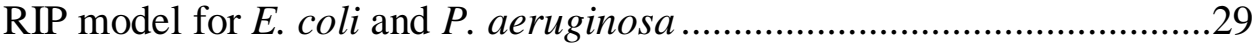

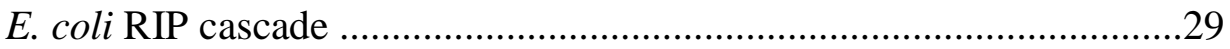

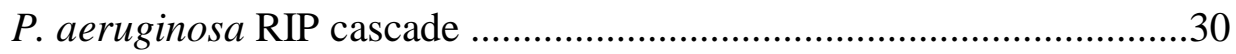

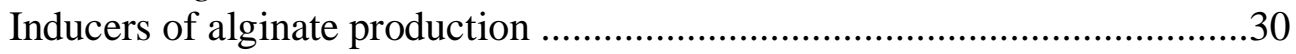

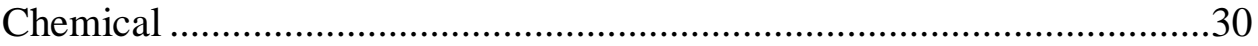

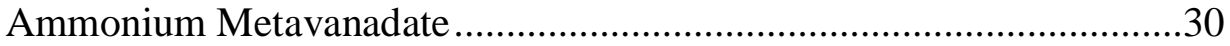

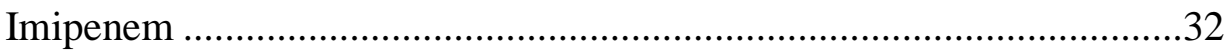




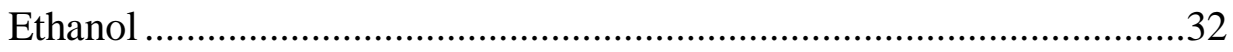

Phage

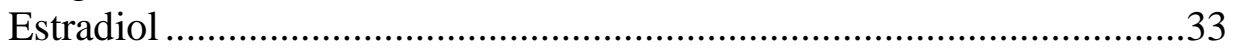

Mucoid phenotype and antibiotic resistance of $P$. aeruginosa .........................33

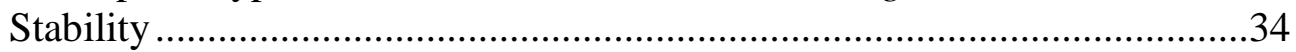

Strategy to map suppressor of alginate production mutants ...........................35

Cosmid-based technique ........................................................................35

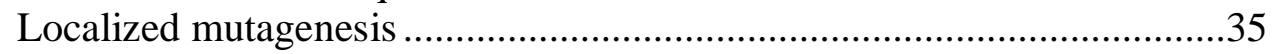

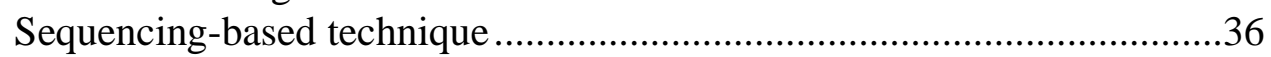

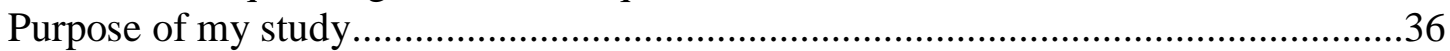

i. Specific aim 1: To map the genes on cosmid PMO012217 involved in

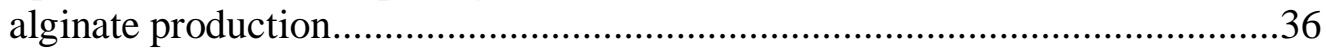

ii. Specific aim 2: To determine mechanism of action of LptD ............................37

iii. Specific aim 3: To identify role of PLP in virulence of P. aeruginosa ............37

CHAPTER 2

Outer membrane protein LptD (PA0595) plays a role in the regulation of alginate

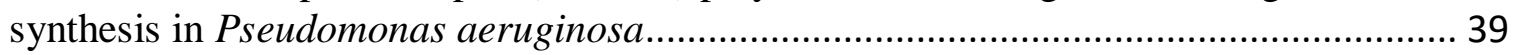

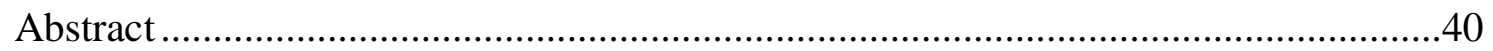

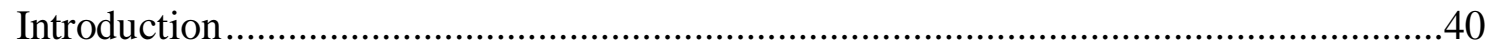

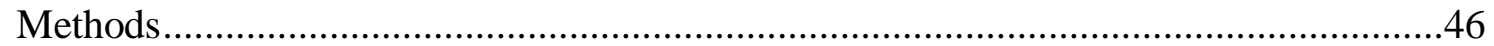

Bacterial strains, plasmids, media and primers.........................................................46

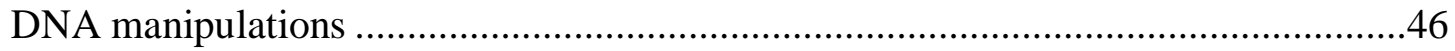

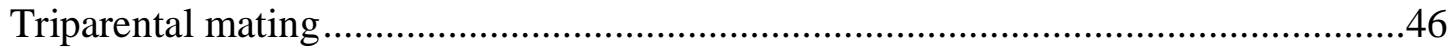

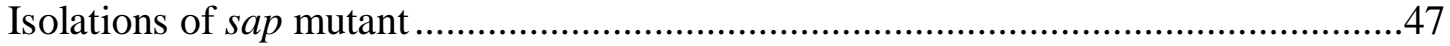

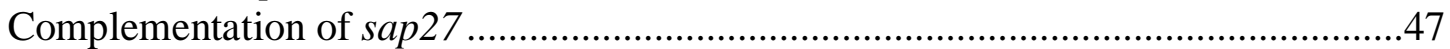





Construction of plasmids containing the ORF $\operatorname{lptD}$-operon, surA, $p d x A$, optP …........53

Construction of plasmid containing lptD-surA-lptE ……….....................................54





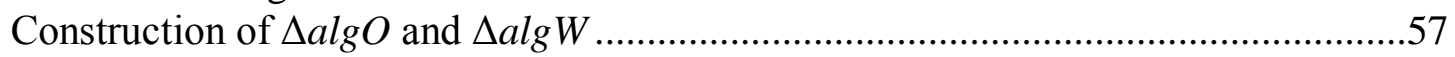

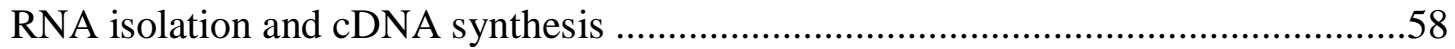

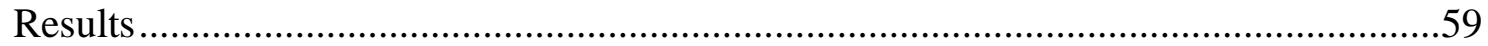

Identification of the sap27 complementing cosmid.................................................59

Mapping the gene in the complementing cosmid pMO01227 using transposon..........60



Co-expression of lptD-surA-lptE in sap27 ........................................................64

Expression of optP encoding a large OMP in sap27 ...............................................66

Mapping of mutation in sap27 ...........................................................................67

Increased expression of lptD-surA-lptE in $\triangle a \lg O$ and $\triangle a l g W$ mutant

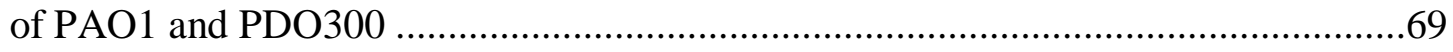

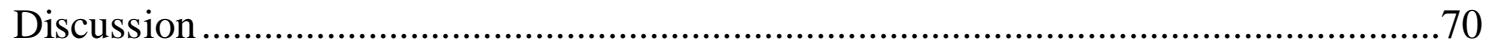

Outer membrane protein LptD regulates alginate production ......................................71 


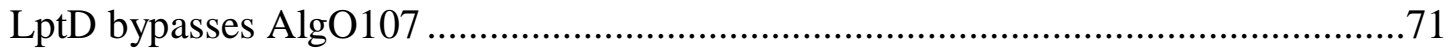

LptD influences membrane of RIP cascade ............................................................72

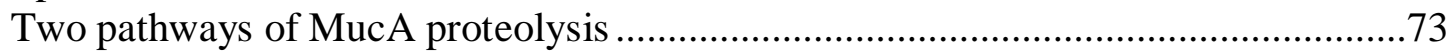

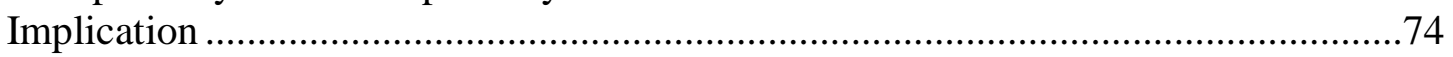

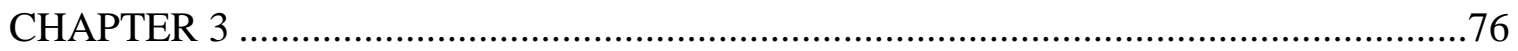

Pyridoxal 5'-phosphate (PLP) enhances pyocyanin biosynthesis in

Pseudomonas aeruginosa .................................................................................... 76

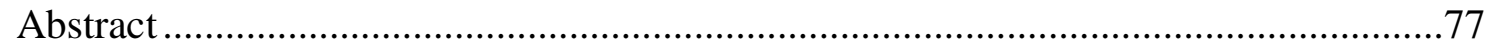

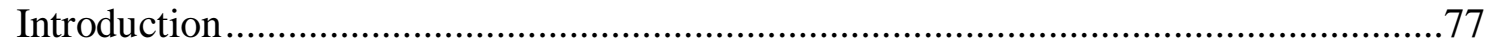

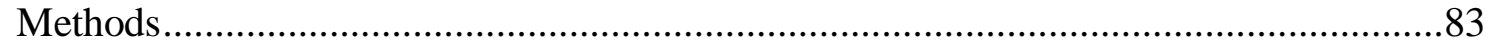

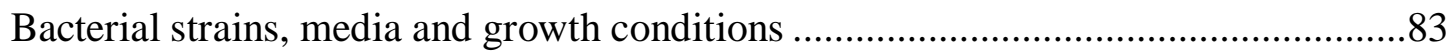





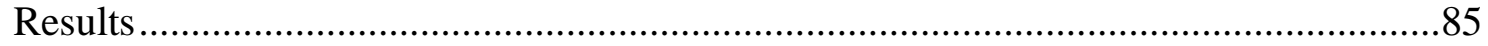

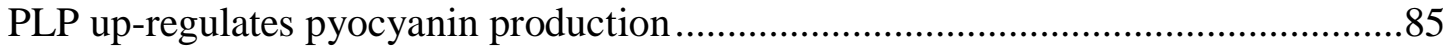

Pyocyanin production in presence of aromatic amino acids .........................................86

PLP increases the transcription of pyocyanin biosynthesis operon .............................87

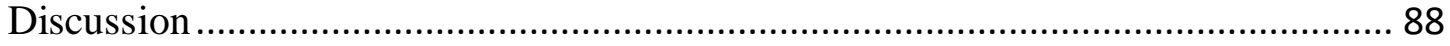

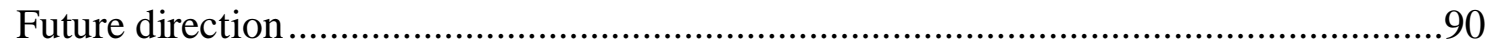

Construction of a conditional $p d x A$ mutant ...............................................................90

qPCR analysis of the first genes in the pyocyanin biosynthesis pathways ...................91

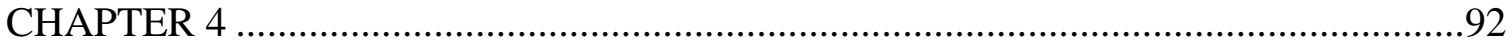

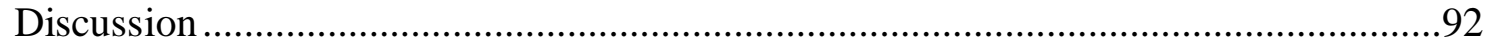

Outer membrane protein LptD .............................................................................95

The role of the second gene of the lptD-operon, surA, in LptD translocation.............97

Role of LptE in the proper folding of LptD in the outer membrane ...........................98



Role of LptD in alginate production: a potential model ..........................................101

Role of PLP in pyocyanin production in P. aeruginosa ...........................................101

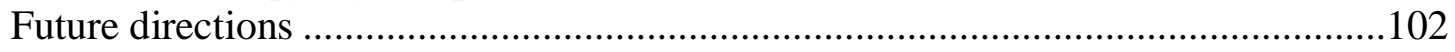

Final conclusion and implications ........................................................................107

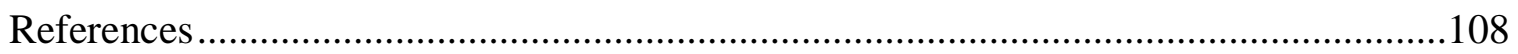

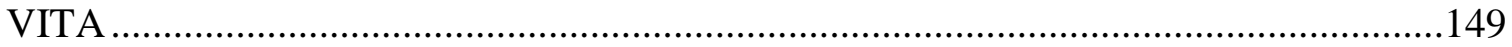




\section{LIST OF TABLES}

TABLE TITLE

PAGE

NUMBER

$\begin{array}{ll}1.1 & \text { Genes associated with alginate biosynthesis }\end{array}$

1.2 List of proteins involved in RpoE regulation 24

1.3 Comparison of E. coli and P. aeruginosa homologs 25 involved in the anti-sigma factor (RseA and MucA, respectively) proteolysis

2.1 Bacterial strains and plasmids used in this study 47

$\begin{array}{lll}2.2 & \text { Primers used in this study }\end{array}$

3.1 Predicted ORFs that requires vitamin $\mathrm{B}_{6}$ as a co-factor $\quad 78$

$\begin{array}{lll}3.2 & \text { Primers used for qPCR } & 84\end{array}$ 


\section{LIST OF FIGURES}

FIGURE TITLE

PAGE

NUMBER

1.1 An electron micrograph of $P$. aeruginosa

3

1.2 Classification of CF mutants based on their cellular

9 phenotype

1.3 The most common microbes found in the lung of CF $\quad 10$

1.4 Common mutations in P. aeruginosa CF isolates 12

$\begin{array}{lll}1.5 & \text { Alginate composition } & 13\end{array}$

1.6 A proposed model of alginate biosynthesis 16

1.7 Proposed model for the anti-sigma factor proteolysis in $E . \quad 31$ coli and P. aeruginosa

1.8 Pyocyanin production pathway in P. aeruginosa 37

2.1 Genetic map of cosmid pMO012217 and lptD-operon $\quad 60$

2.2 Verification of $l p t D$ is a part of a three-gene operon 62

2.3 Identification and mapping of the gene responsible for 63 restoring mucoid phenotype

2.4 Alginate levels in the presence of increased expression of 65 lptD

2.5 Cellular localization of LptD 66

2.6 Mapping the algO mutation in sap27 $\quad 67$ 
2.7 Model for mucoid conversion due to increase expression of 73 lptD in $\mathrm{mucA}^{+}$(PAO1) and $m u c A 22$ (PDO300) backgrounds

$\begin{array}{lll}3.1 & \text { Synthesis of pyocyanin from chorismic acid }\end{array}$

3.2 Detail pathway for the synthesis of pyocyanin 82

3.3 Different forms of $\mathrm{B}_{6}$ vitamers 83

3.4 Pyocyanin production in the presence of PLP and aromatic 86 amino acids

3.5 Expression of genes in phz-operon due to PLP 87

3.6 Strategy to construct $p d x A$ conditional mutant of $P$. aeruginosa 


\section{LIST OF SYMBOLS}

$\begin{array}{cl}\text { Symbol } & \text { Term/unit } \\ \alpha & \text { Alpha } \\ \beta & \text { Beta } \\ \sigma & \text { Sigma } \\ \Delta & \text { Delta (deletion) } \\ { }^{\circ} \mathrm{C} & \text { Degree Celsius } \\ \mu \mathrm{g} & \text { Microgram } \\ \mu \mathrm{L} & \text { Microliter } \\ \% & \text { Percentage }\end{array}$




\section{LIST OF ABBREVIATIONS/ACRONYMS}

Term

Adenosine triphosphate

Alginate

Base pair

Basic local alignment search tool

bis-(3'-5')-cyclic-guanosine monophosphate

Community-acquired pneumonia

Cystic Fibrosis

Cystic Fibrosis Transmembrane Conductance

Regulator gene

Cystic Fibrosis Transmembrane Conductance Regulator protein

Deoxyribonucleic acid

Ethylenediaminetetraacetic acid

Extracytoplasmic function

Forced expiratory volume

Gravitational force

Hour

Luria Bertani

Lipopolysaccharide

Lipopolysaccharide transport protein D gene

Lipopolysaccharide transport protein D protein

Milliliter

Mucoid
Abbreviation/Acronyms

ATP

Alg

bp

BLAST

c-di-GMP

CAP

CF

$c f t r$

CFTR

DNA

EDTA

ECF

FEV

$g$

$\mathrm{hr}$

LB

LPS

lptD

LptD

$\mathrm{mL}$

Muc 
Sodium chloride

Outer membrane

Open reading frame

Pseudomonas isolation agar

Pyridoxal 5'-phosphate

Polypeptide transport-associated

Polymerase chain reaction

Pseudomonas quinolone signaling

Quantitative PCR

Quorum sensing

Regulated intramembrane proteolysis

Regulator of sigma factor $\mathrm{E}$

RNA polymerase sigma-E factor

Transmembrane helix prediction hidden Markov model
$\mathrm{NaCl}$

$\mathrm{OM}$

ORF

PIA

PLP

POTRA

PCR

PQS

qPCR

QS

RIP

Rse

RpoE

TMHМM 


\section{CHAPTER 1}

Posttranslational Regulation of Antisigma Factors of RpoE: A Comparison Between the Escherichia coli and Pseudomonas aeruginosa

Part of this introduction is published as a chapter in a book "Stress and environmental regulation of gene expression and adaptation in bacteria” Wiley Blackwell publication Sundar Pandey, Kyle Martins, and Kalai Mathee

DOI: $10.1002 / 9781119004813 . c h 32$ 


\begin{abstract}
Pseudomonas aeruginosa, a rod-shaped Gram-negative bacterium, is an opportunistic human pathogen associated with skin and soft tissue infections, sepsis, and nosocomial infection. The bacterium can establish chronic infection and causes demise of people with cystic fibrosis. One of the virulence factors of the $P$. aeruginosa is an exopolysaccharide alginate. Alginate is synthesized in response to environmental conditions. The biosynthesis of alginate in P. aeruginosa is a highly regulated process where an extracytoplasmic sigma factor AlgT/U plays a key role. The increased alginate producing phenotype of $P$. aeruginosa is known as mucoid phenotype. The mechanism of mucoid phenotype in $P$. aeruginosa is extensively studied where non-mucoid to mucoid transition is the result of the mutation of inner membrane protein MucA. The degradation of MucA is a sequential process which is known as regulated intramembrane proteolysis (RIP). The RIP mechanism is well conserved in Gram-negative bacteria. This chapter describes the RIP cascade in P. aeruginosa and compares it with Escherichia coli.
\end{abstract}

\title{
Pseudomonas aeruginosa
}

In 1882, Carle Gessard isolated an organism that causes a blue-green coloration of wound dressings and named the microbe as Bacillus pyocyaneus, which is now known as P. aeruginosa (Gessard, 1882; Webster and Bernheim, 1936). The P. aeruginosa is a Gram-negative, rod-shaped (Figure 1.1) measuring $0.5-0.8 \mu \mathrm{m}$ by $1.5-3.0 \mu \mathrm{m}$, typically with monotrichous flagellum and multiple pili for locomotion. 
P. aeruginosa is a biochemically versatile microorganism that can adapt to different ecological niches ranging from soil, water, plants and humans (Holloway, 1955; Rahme et al., 1995). In hospitals, the bacterium can colonize sinks, humidifiers, respirators and sometimes it can be found on the hands of medical personnel (Aiello et al., 2003; Hota et al., 2009; Jadhav et al., 2013). P. aeruginosa is an aerobic gammaproteobacterium but it can grow in oxygen-limited conditions by using nitrate or arginine as the terminal electron

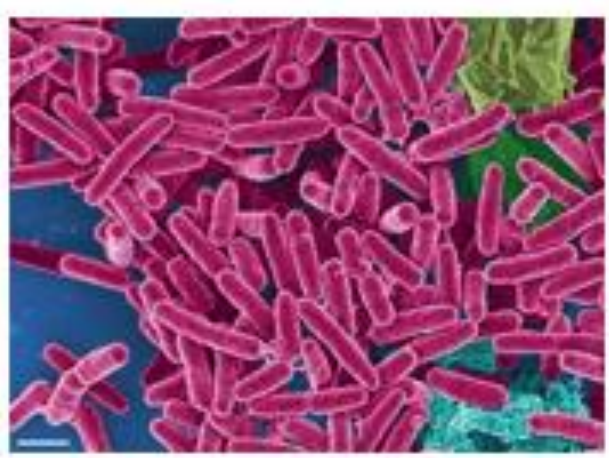

Figure 1.1. An electron micrograph of $P$. aeruginosa. (Courtesy: http://www.waterscans.rs/)

acceptor (van Hartingsveldt and Stouthamer, 1973). P. aeruginosa does not usually establish infection in normal humans but the bacterium can become an opportunistic pathogen and establish disease condition in patients with impaired host defense. It can cause both acute and chronic infection.

\section{Acute infection by $P$. aeruginosa}

i. Acute pneumonia in hospitalized and in particularly mechanically ventilated patients

P. aeruginosa along with Staphylococcus aureus are the most common bacteria that cause ventilator-associated pneumonia with a high mortality rate of $13.5 \%$ 
(Rello et al., 1996). P. aeruginosa can contribute to community-acquired, hospital-acquired, and healthcare-associated pneumonia (Fujii et al., 2014). $P$. aeruginosa is associated with $92 \%$ of community-acquired pneumonia (CAP) with a mortality rate of $33 \%$ (Hatchette et al., 2000). In the case of CAP, younger patients recover better than older patients. In fatal cases, patients die

because of septic shock due to organ failure (Huhulescu et al., 2011; Maharaj et al., 2017). In cases of recovery, the complications could still arise resulting in parenchymal scarring and recrudescence, which require repeated antibiotic treatment (Maharaj et al., 2017).

\section{ii. Sepsis in patients with extensive burns}

Fever diarrhea, pneumonia, skin lesion, and shock are the most common symptom of sepsis caused by $P$. aeruginosa (Grisaru-Soen et al., 2000; Huang et al., 2002). The mortality rate of $P$. aeruginosa associated sepsis in children ranges from 20\% - 50\% (Huang et al., 2002).

\section{iii. Corneal infection in individuals wearing contact lenses}

Gram-negative bacteria and fungi are the dominant pathogens of corneal infection in tropical or sub-tropical climates (Lichtinger et al., 2012; Stapleton et al., 2012). P. aeruginosa is the most common organism in contact lensrelated disease (Stapleton et al., 2012). It has been proposed that the lens, storage case, and ocular environment provide a favorable niche for the survival of P. aeruginosa (Stapleton et al., 2012). It has been shown that exoU expressing phenotype is highly selected in contact-lens related corneal keratitis compared to non-contact lens-related keratitis (Choy et al., 2008). 
iv. Urinary tract infection in patients with indwelling foley catheters

P. aeruginosa causes biofilm-mediated infections that includes catheterassociated urinary tract infections, ventilator-associated pneumonia, infections related to mechanical heart valves, stents, grafts, and sutures (Bharathi et al., 2014; Bouchart et al., 1997; Chan et al., 2018; Dapas et al., 2016; Edmiston et al., 2013; Olejnickova et al., 2014). P. aeruginosa is responsible for $12 \%$ of all nosocomial urinary tract infections (Cole et al., 2014).

v. Bacteremia and sepsis in immunocompromised patients, particularly neutropenic patients receiving cytotoxic therapies

A positive correlation has been shown between $P$. aeruginosa and neutropenic patients (Lee et al., 2011; Lucas et al., 2017; Righi et al., 2017).

\section{vi. Post-surgical wound infection}

Post-surgical wound infection occurs after surgical operation, may originate during the operation or may occur after the operation from sources in the ward or as a result of some complications (Mousa, 1997). The increase rate of $P$. aeruginosa infection has been associated in post-operative wound infections (McNeil et al., 2001; Ranjan et al., 2010)

\section{vii. Burn wound infection}

P. aeruginosa is the most commonly isolated from burn wounds where the organism can cause sepsis (Glik et al., 2017; Hsueh et al., 1998). 


\section{Chronic infection by $P$. aeruginosa}

Chronic infection is defined as an infection that persists in spite of therapy, and the host's immune and inflammatory response (Pressler et al., 2011b). The common chronic lung infections caused by $P$. aeruginosa are in the lung of patients with chronicbronchiectasis, chronic obstructive pulmonary disease (COPD), and cystic fibrosis (CF) (Finch et al., 2015; Hilliam et al., 2017; Lieberman and Lieberman, 2003; McAvoy et al., 1989). CF and COPD have different causal origins, but they share the same relevant symptoms and bacterial colonization. The difference between CF and COPD patients is that the $P$. aeruginosa infections occur earlier in the case of the CF (Martinez-Solano et al., 2008).

According to Leed's criteria, chronic $P$. aeruginosa infection in $\mathrm{CF}$ patients is defined when $>50 \%$ of sputum cultures are positive for $P$. aeruginosa in the preceding 12 months of routine checkups (Lee et al., 2003). But the Leed's criteria are difficult to implement especially in young children because of the requirement of sputum samples and follow-up time. To solve the issue of sputum culture of $P$. aeruginosa it was hypothesized that $P$. aeruginosa chronic infection is associated with the dominance of the bacterium in the lungs while intermittently infected patients only showed low abundance of the pathogen. The qPCR technique can be used as good diagnostic tool to discriminate between intermittent and chronic infection (Boutin et al., 2018). It has been accepted that $10^{3.4}$ colony-forming units (CFU) of $P$. aeruginosa as a chronic infection in the lung of $\mathrm{CF}$ patients and qPCR value for the bacterium is adjusted according to the CFU (Boutin et al., 2018). 


\section{Cystic Fibrosis}

Cystic Fibrosis $(\mathrm{CF})$ is a debilitating autosomal recessive genetic disease that affects multiple organs resulting in exocrine pancreatic insufficiency in diabetes, malnutrition and impaired growth associated with gastrointestinal malabsorption, male infertility from congenital malformation of the vas deferens, and sinusitis (Coste et al., 1995; Dray et al., 2005; Yu et al., 2012). CF is distributed worldwide (Farrell, 2008b; Prasad et al., 2010; Silva Filho et al., 2016; Stewart and Pepper, 2016). On the basis of live births, the highest incidence of the $\mathrm{CF}$ disease is found in Brazil especially in Euro Brazilians (1/1600) and the lowest incidence is found in the Japanese population (1/100,000-350,000) (Faucz et al., 2010; Yamashiro et al., 1997). The prevalence of the $\mathrm{CF}$ in the United States is one in 3500 and in the European Union is one in 2500 (Farrell, 2008a). Currently, 70,000 CF patients have been registered worldwide and 30,000 in the United States (2016).

Sweat glands microdissected from the skin of CF patients showed a low permeability of chloride ions leading to poor reabsorption of $\mathrm{NaCl}$ resulting in high $\mathrm{NaCl}$ concentrations in the sweat (Quinton, 1983). The inability of the sweat duct to re-absorb $\mathrm{NaCl}$ led to the identification of a defective locus on chromosome seven (Knowlton et al., 1985; Tsui et al., 1985; Wainwright et al., 1985). Using the chromosome walking and jumping technique, approximately 250,000 base pairs of genomic DNA encoding the cystic fibrosis transmembrane conductance regulator (CFTR), a cAMP-regulated chloride ion channel, was identified (Riordan et al., 1989; Rommens et al., 1989). Expression of the cftr gene in recombinant baculovirus led to the confirmation of the role of CFTR in anion channel regulation (Kartner et al., 1991). 
The lack of functional CFTR at the apical membrane of a secretory epithelial cell results in defective $\mathrm{Cl}^{-}$and bicarbonate secretion, increase $\mathrm{Na}^{+}$absorption and mucus secretion that leads to dehydration and acidification of the airway surface liquid (Chen $e t$ al., 2010; Darch et al., 2015; Tang et al., 2012). These impaired mucociliary clearance that provokes recurrent infection and uncontrolled inflammation resulting in lung damage, the primary cause of morbidity and mortality of people with CF (Boucher, 2007; Stoltz et al., 2015).

The CFTR protein is composed of 1,480 amino acids (aa) and 2026 different $c f t r$ mutations are classified (Riordan et al., 1989). Classification of cftr mutation on the basis of therapeutic outcome is recently proposed (De Boeck and Amaral, 2016; Marson et al., 2016). Based on clinical features classes I, II, and III are classified as more-severe disease whereas classes IV, V, and VI are grouped as less-severe (Marson et al., 2016). But for simplicity, this dissertation adopts the cftr mutation based on the biological defect (Figure 1.2).(Veit et al., 2016).

1. The Class I defect results from frameshift, splicing, or non-sense mutations that introduce premature termination codons which cause the expression of CFTR to be severely reduced or absent.

2. Class II defects encompass mutations that lead to misfolding and/or premature degradation in the endoplasmic reticulum. The defective protein biogenesis causes a highly reduced number of CFTR molecules on the secretory epithelial cell surface (Bombieri et al., 2015). 
3. Class III mutants have abnormal gating that is characterized by reduced open probability (CFF, 2011; Marson et al., 2016; Veit et al., 2016).

4. Class IV mutant of CFTR has altered channel conductance because of impedance in the ion conduction pore ultimately leading to a reduced unitary conductance (CFF, 2011; Hammerle et al., 2001; Marson et al., 2016; Sheppard et al., 1993; Veit et al., 2016).

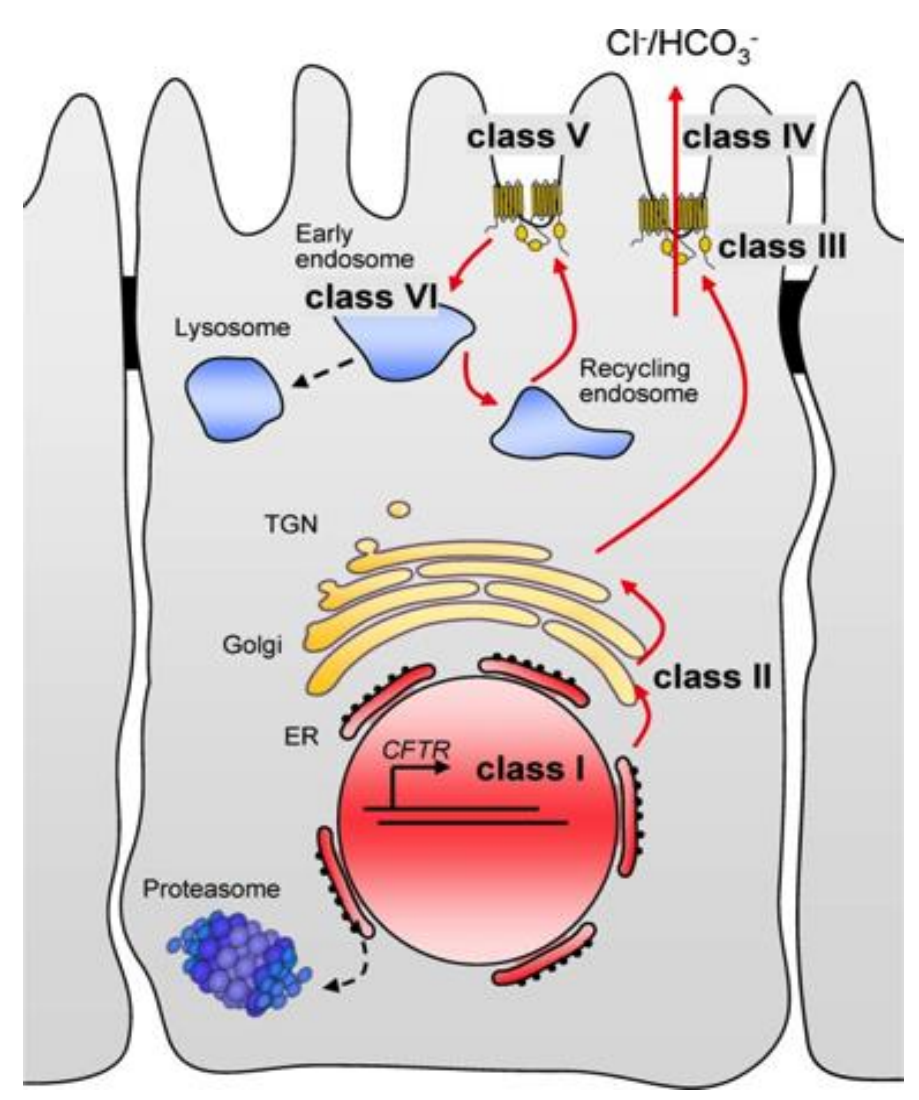

Figure 1.2. Classification of CF mutations based on their cellular phenotype. Class I: protein synthesis defect; class 11: maturation defect; class 11l: gating defect; class IV: conductance defect; class V: reduced quantity; and class Vl: reduced stabitlity. ER, endoplasmic reticulum; TGN, trans-Golgi network. Courtesy (Veit et al., 2016) 
5. Class V type CFTR has altered promoter or splicing abnormalities resulting in protein abundance that do not change the conformation (CFF, 2011; Haardt et al., 1999; Highsmith et al., 1997; Marson et al., 2016; Silvis et al., 2003; Veit et al., 2016; Zielenski and Tsui, 1995).

6. Class Vl a mutation, which reduces the conformational stability of the CFTR in post-endoplasmic reticulum and/or at the plasma membrane (Sheppard et al., 1993:Hammerle, 2001 \#592). In addition, class VI type mutation generates additional internalization signals. Because of defective $c f t r$ gene, dehydrated mucus layer builds up in an epithelial layer that impairs mucociliary clearance that is a characteristic feature of patients with CF (Cohen and Prince, 2012). The lack of mucociliary clearance causes the gradual thickening of mucus, which eventually leads to the mucus plugs formation. The mucus plugs not only

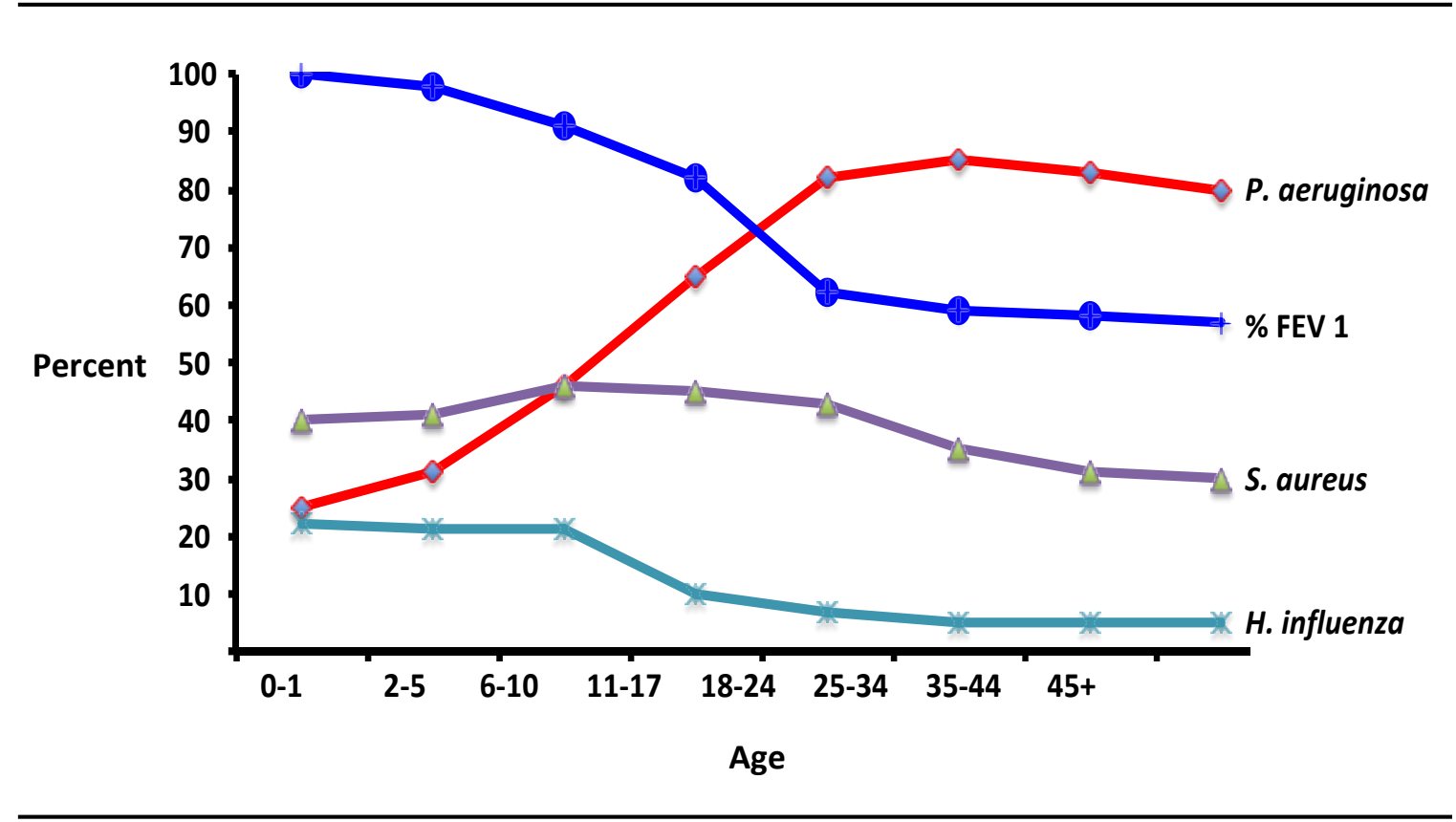

Figure 1.3. The most common microbes found in lung of CF. Relationship shows inverse relationship between mucoid $P$. aeruginosa and lung function predicted by forced expiratory volume 1 (FEV1). FEV1 indicates severity in lung function. Courtesy (Pier, 2000). 
obstruct the airways but also create oxygen gradient from hypoxic to anoxic regions (Worlitzsch et al., 2002). The environment is made further anoxic because of the growth of $P$. aeruginosa where the bacterium uses nitrates and nitrites as terminal oxygen accepter (Worlitzsch et al., 2002). Furthermore, $P$. aeruginosa decreases the apical expression of cftr gene (MacEachran et al., 2007; Rubino et al., 2014; Trinh et al., 2015). Oxygen availability, inflammation, and microbial communities are heterogeneous in CF lung that affects the host microbial interaction and eventually the disease progression.

\section{Microbial communities in CF airways}

The Streptococcus, Veillonella, and Prevotella are the dominant genera in a healthy lung (Li et al., 2016). But, bacterial species like Haemophilus influenzae, S. aureus, P. aeruginosa, Burkholderia cepacia, Alcaligenes xylosoxidans, Klebsiella spp., some pathogenic virus like respiratory syncytial virus and adenoviruses, and the fungi Aspergillus and Candida species are commonly found in CF airways (Haase et al., 1991; Lambiase et al., 2006; Nixon et al., 2001; Scoffone et al., 2017; van Ewijk et al., 2008; Willger et al., 2014). In CF pediatric samples, Streptococcus, Haemophilus, Staphylococcus, and Achromobacter predominant whereas and in adult Pseudomonas, Burkholderia, Streptococcus, Haemophilus, and Staphylococcus are the dominant genus. P. aeruginosa becomes dominant pathogen as the increase in the age of people with CF (Figure 1.3). Rothia, Preovtella, Gemella, Actinomyces, and Veillonella are prevalent in both pediatric and adult samples (Coburn et al., 2015). One of the methods that is used to assess the lung disease status of the individual with cystic fibrosis is forced expiratory 
volume in one sec (FEV1). FEV1 is the quantity of air that can be forcibly exhaled from the lungs in first one second. The ratio of FEV1 of CF individual and FEV1 of reference population is \%FEV1 that indicates the health status of a lung of people with CF (Wang et $a l ., 1993)$. It was observed that the FEV1 ratio of individual with $\mathrm{CF}$ is lower indicating the decline function of the lung (Pier, 2000)(Figure 1.3).

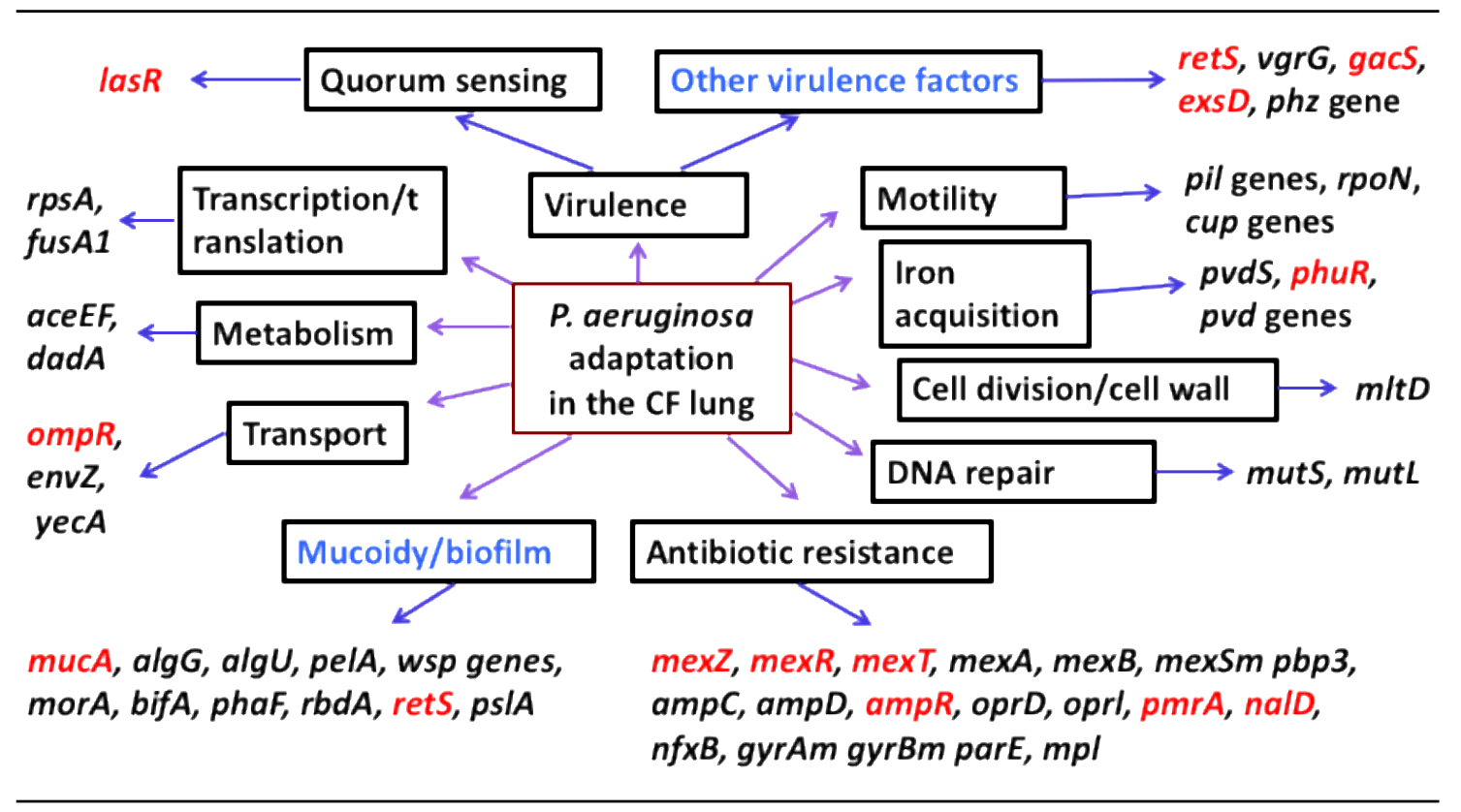

Figure 1.4. Common mutations in $\boldsymbol{P}$. aeruginosa CF isolates. Genes encoding regulatory proteins are highlighted in red. The phenotype that is of interest of this dissertation is shown in box with blue color. Courtesy (Winstanley et al., 2016)

\section{Genotypic change in $P$. aeruginosa in CF lung}

$P$. aeruginosa, during infection of $\mathrm{CF}$ airways, undergo evolution and adaptation (Figure 1.4) (Ciofu et al., 2010). The adaptive phenotypes include increased alginate production, loss of quorum-sensing, motility, effector proteins of the type lll secretion system, and loss of the O-antigen component of the lipopolysaccharide, reduce virulence, reduced capacity of the non-mucoid phenotype for in vitro biofilm formation (Cigana et 
al., 2016; Ciofu et al., 2010; Cullen et al., 2015; Oliver et al., 2000; Smith et al., 2006; Warren et al., 2011; Williams et al., 2018; Workentine et al., 2013). It is important to understand that both environmentally-acquired strains and patient-to-patient transmissible strains of $P$. aeruginosa appear to have a similar genomic mutation that provides an adaptive advantage in the lung of CF patient (Fothergill et al., 2012; Jelsbak et al., 2007; Williams et al., 2018). One study showed that the genotypic and phenotypic diversity could also be the result of recombination and not always the result of the spontaneous mutations occurs in the P. aeruginosa (Darch et al., 2015).

One of the genotypic and phenotypic changes that is of interest of this dissertation is an emergence of mucoid colonies caused by increased production of alginate (Carlson and Matthews, 1966). Along with alginate two other polysaccharide (Pel and Psl) play a role in the development and structural maintenance of biofilm of P. aeruginosa (Colvin et al., 2012; Stapper et al., 2004).



$\beta$-D-ManUA-(1->4)-3-0-acetyl- $\beta$-D-ManUA-(1->4)-2-0-acetyl- $\beta$-D-ManUA-(1->4)- $\beta$-L-GulUA-(1->4)-2-0-acetyl- $\beta$-D-ManUA

Figure 1.5. Alginate composition. The O-acetylation occurs in mannuronic acid. The $\beta$ 1,4-glycosidic bond link the guluronic and mannuronic acid. Courtesy (Franklin et al., 2011) 


\section{Alginate}

Alginate is an exopolysaccharide, meaning it is not covalently linked to the bacteria, composed of non-repeating unit of O-acetylated $\beta$-D-mannuronic (M) acid residues randomly interspersed with its $\mathrm{C}^{\prime}$ ' epimer $\alpha$-L-guluronic acid (G) residues by 1,4glycosidic bonds (Figure 1.5)(Chitnis and Ohman, 1990; Schurks et al., 2002; Skjakbraek et al., 1986). The alginate of $P$. aeruginosa is composed of random blocks of MM and MG residues. In $P$. aeruginosa FRD1, a sputum mucoid isolate from a CF patient, the molar ratio of mannuronic acid and guluronic acid was found to be 2:1 (Schurks et al., 2002). Acetylation of alginate promotes the cell aggregation, confers elasticity, and provides resistance to the opsonic killing of $P$. aeruginosa (Moradali et al., 2015; Pier et al., 2001; Wloka et al., 2004). Alginate is not required but it affects the formation of biofilm (Hentzer et al., 2001; Stapper et al., 2004). Antibiotics, high osmolarity, dehydration, and release of hydrogen peroxide by polymorphonuclear cells have all been shown to promote alginate production (Berry et al., 1989; Devault et al., 1990; Govan and Fyfe, 1978; Mathee et al., 1999). Alginate protects $P$. aeruginosa from an oxidative burst from the immune system; provides an advantage for adherence to an extracellular material, antibiotic tobramycin, and protection from phagocytes (Hentzer et al., 2001; Leid et al., 2005; Tielen et al., 2005). It has been shown that alginate-deficient $P$. aeruginosa develops biofilm but with a reduced number of viable cells compared to alginate-producing P. aeruginosa (Ghafoor et al., 2011). Free radicals produced by macrophages and neutrophils cannot depolymerize nor is the alginate degraded during phagocytosis suggesting alginate is helpful for bacterial survival in CF lung (Simpson et al., 1989). It is interesting that the mucoid P. aeruginosa isolated from chronic $\mathrm{CF}$ adults is less resistant than non-mucoid isolated against 
antibiotics colistin, tobramycin, meropenem, and ciprofloxacin suggesting the presence of a secondary mutation.

The high amount of immunoglobulin $\mathrm{G}(\mathrm{IgG})$ is mounted against the alginate in chronically affected CF patients but such IgG is ineffective to clear P. aeruginosa infection (Mauch et al., 2017; Meluleni et al., 1995).

\section{Biosynthesis of alginate}

Alginate biosynthesis requires multiple gene products (Figure 1.6) (Table 1.1). The algD operon (algD8alg44algKalgEalgGalgXalgLalgIalgJalgFalgA) and other gene, algC, which is not encoded in the $\operatorname{alg} D$ operon, are required for alginate biosynthesis in $P$. aeruginosa (Table 1.1) (Chitnis and Ohman, 1993; Shortridge et al., 1991). For alginate biosynthesis, the carbon is funneled from fructose 6-phosphate that is converted to mannose 6-phosphate by AlgA (Darzins et al., 1986; Shinabarger et al., 1991). Then, AlgC,

a phosphomannomutase isomerizes mannose 6-phosphate to mannose 1-phosphate (Zielinski et al., 1991). Other protein AlgA then converts mannose 1-phosphate to GDPmannose by AlgA (May et al., 1994). Then, AlgD oxidizes GDP-mannose to GDPmannuronate (Roychoudhury et al., 1989). For the synthesis of alginate, D-mannuronic acid is polymerized by the inner membrane protein Alg8, and copolymerase, Alg44 (Merighi et al., 2007; Remminghorst et al., 2009; Remminghorst and Rehm, 2006). Then $\mathrm{A} \lg \mathrm{I}, \mathrm{Alg} \mathrm{J}$, and $\mathrm{AlgF}$ play roles in the acetylation of the polymer (Franklin and Ohman, 1996; Shinabarger et al., 1993). The O-acetylation of alginate occurs only on mannuronate residues (Davidson et al., 1977). 


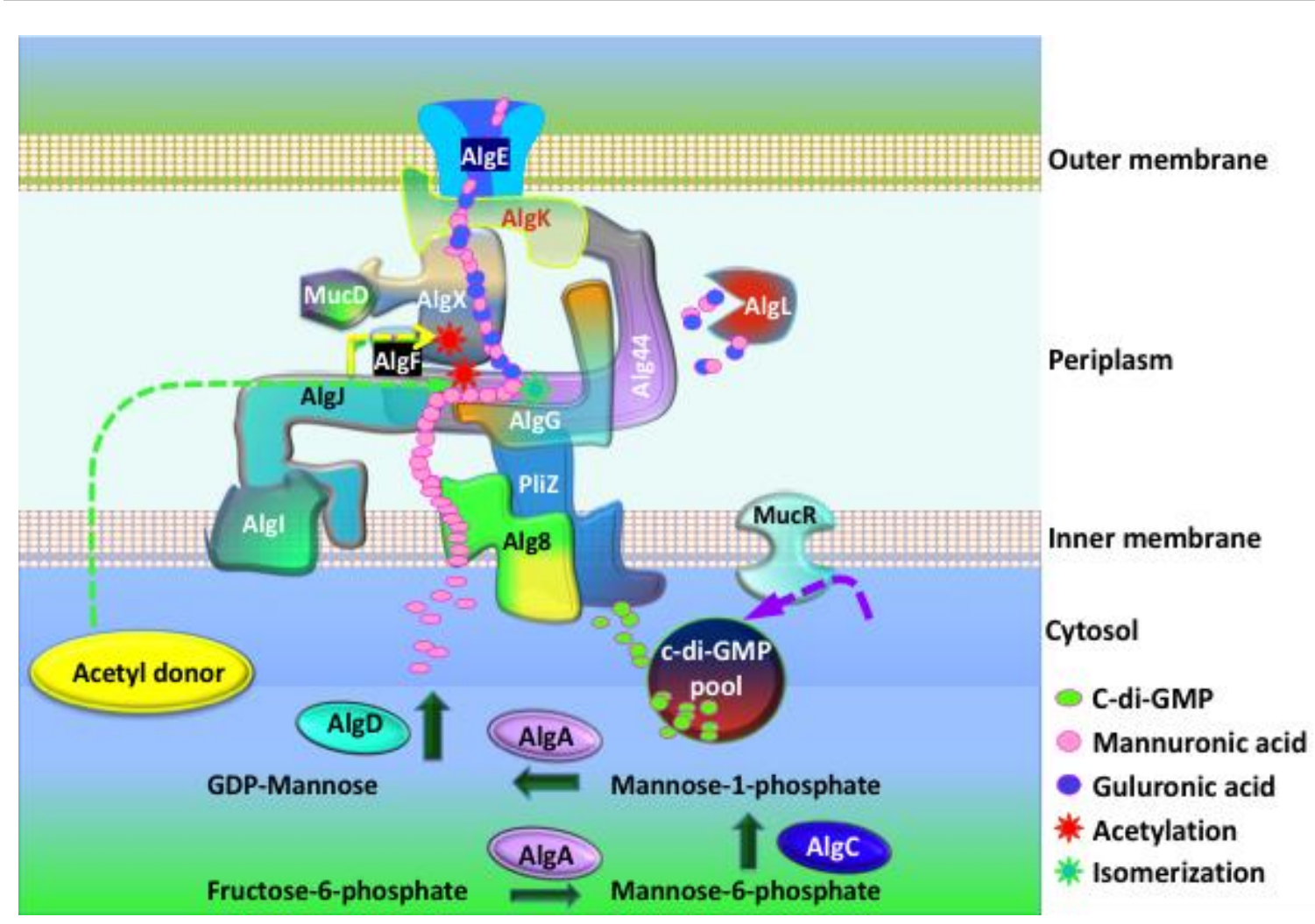

Figure 1.6. A proposed model of alginate biosynthesis. AlgA, AlgC, and AlgD construct the alginate monomers in the cytosol of the P. aeruginosa. Polymerization, acetylation, and epimerization occur in periplasm, and $\mathrm{AlgE}$ is responsible for exporting the polymer to the extracellular environment (Franklin et al., 2011; Moradali et al., 2015; Ramsey and Wozniak, 2005).

After acetylation of the mannuronate residues, the AlgG epimerizes the nonacetylated D-mannuronic acid residues into the L-guluronic acid (Franklin et al., 1994). Still, it is not known whether epimerization occurs prior to or after acetylation. Physiologically the acetylation of alginate increases the overall gel volume that might protect $P$. aeruginosa from dehydration (Skjakbraek et al., 1989). Immunologically Oacetylation of alginate protects the bacterium from neutrophils and lymphocytes (Mai et al., 1993). 


\section{Regulation of alginate biosynthesis in $P$. aeruginosa}

The 12 gene $\operatorname{alg} D$ operon is positively regulated by an alternate sigma factor AlgT/U (Wozniak and Ohman, 1994). The algD promoter contains a large 367-bp leader region between the transcription start site and translation start codon (Wurtzel et al., 2012). The direct SELEX enrichment approach showed AlgB binds 50 bp located at -274 to -224 upstream relative to $\operatorname{alg} D$ transcription site, which is $367 \mathrm{bp}$ upstream of $\operatorname{alg} D$ translation start site (Leech et al., 2008). It has been also shown that AlgT/U activates algR and algB both of which positively regulate $\operatorname{algD}$ transcription (Leech et al., 2008; Wozniak and Ohman, 1994).

Alginate $\mathrm{R}(\mathrm{AlgR})$ is homologous to response regulator that has atypical sensor kinase FimS (formally known as AlgZ) whereas AlgB-KinB forms two-component system (Deretic et al., 1989; Ma et al., 1997a; Yu et al., 1997). AlgR has two major structural domains, an N-terminal CheY-like receiver domain, and carboxyl DNA-binding domain of the LytR/YehT/AgrA family of a transcriptional regulator (Kato and Chakrabarty, 1991; Mohr et al., 1991; Mohr et al., 1992). Overexpression of AlgR represses algD transcription and alginate production (Deretic and Konyecsni, 1989). Both AlgB and AlgR do not require phosphorylation of the conserved aspartic acid residue in the regulatory domain to promote alginate production in mucoid $P$. aeruginosa (Ma et al., 1998). Chromosome immunoprecipitation analysis showed $\mathrm{AlgB}$ directly binds to the $\operatorname{alg} D$ promoter (Leech $e t$ al., 2008). 
Table 1.1 Genes associated with alginate biosynthesis

\begin{tabular}{|c|c|c|}
\hline Gene & Function & References \\
\hline & Alginate biosynthesis & \\
\hline $\operatorname{alg} A$ & $\begin{array}{l}\text { Phosphomannose isomerase and GDP- } \\
\text { mannose pyrophosphorylase activity }\end{array}$ & (Shinabarger et al., 1991) \\
\hline $\operatorname{alg} C$ & $\begin{array}{l}\text { Phosphomannomutase and } \\
\text { phosphoglucomutase activities; involved } \\
\text { in LPS biosynthesis }\end{array}$ & $\begin{array}{l}\text { (Regni et al., 2004; Zielinski et } \\
\text { al., 1991) }\end{array}$ \\
\hline $\operatorname{alg} D$ & GDP-mannose dehydrogenase & $\begin{array}{l}\text { (Snook et al., 2003; Tatnell et } \\
\text { al., 1993) }\end{array}$ \\
\hline $\operatorname{alg} E$ & $\begin{array}{l}\text { Outer membrane, alginate-specific ion } \\
\text { channel responsible for alginate export }\end{array}$ & $\begin{array}{l}\text { (Chu et al., 1991; Grabert et al., } \\
\text { 1990) }\end{array}$ \\
\hline $\operatorname{alg} F$ & $\begin{array}{l}\text { Periplasmic protein involved in alginate } \\
\text { O-acetylation }\end{array}$ & $\begin{array}{l}\text { (Franklin and Ohman, 1993; } \\
\text { Franklin and Ohman, 2002) }\end{array}$ \\
\hline $\operatorname{alg} G$ & $\begin{array}{l}\text { Periplasmic associated, C5-mannuronan } \\
\text { epimerase; protects alginate polymer } \\
\text { from lyase activity }\end{array}$ & $\begin{array}{l}\text { (Franklin et al., 1994; Jain et } \\
\text { al., 2003) }\end{array}$ \\
\hline $\operatorname{algI}$ & $\begin{array}{l}\text { Inner membrane protein involved in } \\
\text { alginate } \mathrm{O} \text {-acetylation }\end{array}$ & (Franklin and Ohman, 2002) \\
\hline algJ & $\begin{array}{l}\text { Membrane-associated, periplasmic } \\
\text { protein involved in alginate O- } \\
\text { acetylation }\end{array}$ & (Franklin and Ohman, 2002) \\
\hline $\operatorname{alg} K$ & $\begin{array}{l}\text { Periplasmic protein required for proper } \\
\text { polymer formation }\end{array}$ & $\begin{array}{l}\text { (Aarons et al., 1997; Jain and } \\
\text { Ohman, 1998) }\end{array}$ \\
\hline $\operatorname{alg} L$ & Periplasmic alginate lyase & $\begin{array}{l}\text { (Monday and Schiller, 1996; } \\
\text { Schiller } \text { et al., 1993) }\end{array}$ \\
\hline $\operatorname{alg} X$ & $\begin{array}{l}\text { Periplasmic protein required for proper } \\
\text { polymer formation }\end{array}$ & $\begin{array}{l}\text { (Monday and Schiller, 1996; } \\
\text { Robles-Price } \text { et al., 2004) }\end{array}$ \\
\hline $\operatorname{alg} 8$ & Membrane protein, alginate polymerase & (Maharaj et al., 1993) \\
\hline $\operatorname{alg} B$ & Positive activator of $\operatorname{alg} D$ transcription & $\begin{array}{l}\text { (Goldberg and Dahnke, 1992; } \\
\text { Wozniak and Ohman, 1991) }\end{array}$ \\
\hline $\operatorname{kin} B$ & $\begin{array}{l}\text { Inner membrane protein; cognate sensor } \\
\text { kinase for } A \lg B\end{array}$ & (Ma et al., 1997b) \\
\hline fimS & $\begin{array}{l}\text { Hypothesized cognate sensor kinase for } \\
\text { AlgR; positively activator of type IV- } \\
\text { mediated twitching motility }\end{array}$ & $\begin{array}{l}\text { (Whitchurch et al., 1996; Yu et } \\
\text { al., 1997) }\end{array}$ \\
\hline
\end{tabular}


$\operatorname{algR} \quad$ Positively activates $\operatorname{alg} C$ and $\operatorname{alg} D$ transcription

$\operatorname{sspA} \quad$ Hypothesized that it reduces transition of RpoD associated genes

RpoN Required for AlgT/U activity in $\operatorname{kin} B$ null mutant
(Deretic et al., 1989;

Nikolskaya and Galperin, 2002)

(Yin et al., 2013)

(Damron et al., 2009)

(Qiu et al., 2008b)

Other regulators of the $\operatorname{alg} D$ operon is alginate and mobility regulator $\mathrm{Z}$ (AmrZ) (Baynham and Wozniak, 1996; Xu et al., 2016). AmrZ has three segments a flexible Nterminus [1-11], a ribbon-helix-helix domain [12-66] and C-terminus [67-108]. Using Chip-Seq and electrophoretic mobility shift assay (EMSA), AmrZ-binding site (ZBS) in $a l g D$ promoter was found around nucleotides -312(ZBS1), -289(ZBS2), -13(ZBS3) and, 75(ZBS4) upstream relative to the $\operatorname{alg} D$ transcription start site of $\operatorname{alg} D$ (Xu et al., 2016). Transcriptional activation of $\operatorname{llg} D$ requires at least 57 bp of DNA between ZBS1 and ZBS2 (Xu et al., 2016). AmrZ binds 8 bp to a direct repeat [GCCATTAC], but a mutation within the repeat results in a four-fold decrease in AmrZ binding and 28 -fold decrease in algD transcription (Baynham and Wozniak, 1996).

Another protein, the integration host factor (IHF) encoded by himA and $\operatorname{him} D$, of P. aeruginosa is an $\alpha \beta$ heterodimer, which through electrophoretic mobility shift assay (EMSA) it was shown that the IHF forms a stable complex with the $\operatorname{alg} D$ promoter (Mohr and Deretic, 1992; Toussaint et al., 1993). There are two IHF binding sites on the algD promoter, one between -80 to -68 and another between 79 to 105 relative to the transcription initiation site of $\operatorname{alg} D$ mRNA. The amount of alginate in IHF mutant cell is half $[200 \mu \mathrm{g} / \mathrm{mg}$ dry cell wt $]$ compared to wild-type $P$. aeruginosa $[400 \mu \mathrm{g} / \mathrm{mg}$ dry cell wt] (DelicAttree et al., 1996). 
Additionally, MucR an inner membrane-anchored protein positively regulates the alginate biosynthesis in $P$. aeruginosa by increasing the level of cyclic-di-GMP in the cytoplasm (Hay et al., 2009).

$\mathrm{Alg} / \mathrm{U}$ regulates the expression of $\operatorname{alg} T, \operatorname{alg} D, \operatorname{alg} R, \operatorname{alg} B, \operatorname{alg} Z, \operatorname{alg} C$ (Devries and Ohman, 1994b; Hershberger et al., 1995; Martin et al., 1994; Wozniak and Ohman, 1994; Zielinski et al., 1991). Alginate production is a metabolically expensive process and to synthesize one set of all proteins for the alginate biosynthesis and production, it requires at least 348,984 GTPs. The required GTP number calculation is only for the protein synthesis. Additionally, the amount of GTP required to the synthesis of alginate molecule itself is yet to be calculated.

Alginate production is energetically expensive thus regulation in alginate production is very controlled transcriptionally by autoregulation of sigma factor AlgT/U and post transcriptionally by sequestration of AlgT/U by anti-sigma factor MucA. The release of $\mathrm{AlgT} / \mathrm{U}$ from MucA is by the process known as regulated intramembrane proteolysis (RIP) that involves series of periplasmic and inner membrane proteases.

In this chapter, genes involved in regulation of alginate production in $P$. aeruginosa are discussed and compared with the genes in E. coli because of the similarity of the pathways between the two bacteria (Ades et al., 1999; Qiu et al., 2007).

\section{Regulated intramembrane proteolysis cascade}

The RpoE homologs function as extracytoplasmic sigma factors (Rowen and Deretic) (Lonetto et al., 1994). The stress signals initiate a proteolytic cascade that eliminates an inner membrane negative-regulator i.e., an anti-sigma factor, freeing RpoE. 
The RIP cascade is common to many bacteria, including Bacillus subtilis (Schobel et al., 2004; Zellmeier et al., 2006), Mycobacterium tuberculosis (Sklar et al., 2010), and Xanthomonas campestris (Bordes et al., 2011). The following section will investigate the role of the major players involved in the P. aeruginosa and E. coli RIP cascade (Table 1.1) and the signals involved. For simplicity, the nomenclature in column 2 and column 6 of the Table 1.2 will be adopted in this chapter. Although the members of the E. coli and $P$. aeruginosa RIP cascade are functionally conserved, there is a wide variation in sequence homology between gene orthologs (Table 1.3)

\section{Extracytoplasmic stress sigma factor $\sigma^{\mathrm{E}}$ : AlgT/U and RpoE}

P. aeruginosa and E. coli RpoE share $66 \%$ homology (Lonetto et al., 1992). E. coli RpoE has been found to restore the mucoid (Alg+) phenotype in $\triangle a \lg T / U$ mutant $P$. aeruginosa (Yu et al., 1995). However, there is no in vivo experimental evidence as to whether algT/U complements the function of $r p o E$ in $E$. coli; although AlgT/U has been shown to transcriptionally activate E. coli RpoE-dependent promoters in vitro (Hershberger et al., 1995). Activation of alternative sigma factors in $P$. aeruginosa and $E$. coli is controlled both at the level of transcriptional initiation and post-translation. The $r p o E$ operon contains four genes, which either positively or negatively regulate its activity in E. coli (Missiakas et al., 1997). The three open reading frames downstream of rpoE encode regulators of sigma $\mathrm{E}$ and are thus named $r s e A, r s e B$, and $r s e C$ (De Las Penas $e t$ al., 1997; Missiakas et al., 1997). In the case of P. aeruginosa, algT/U is a part of a fivegene operon containing algT/U, тисA, тисB, тисC, and mисD (Boucher et al., 1996; Franklin and Ohman, 1996). These Muc proteins regulate AlgT/U activity. 


\section{E. coli RseA and $P$. aeruginosa MucA - the anti-sigma factors}

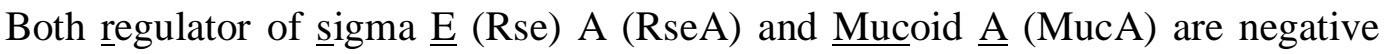
regulators of the alternative sigma factors $\mathrm{RpoE}$ and $\mathrm{AlgT} / \mathrm{U}$, respectively, and are referred to as anti-sigma factors. For more details, analyses of other anti-sigma factors are reviewed previously (Hughes and Mathee, 1998). Though these two proteins are functional equivalents, they share very little sequence homology (Table 1.3). Transposon insertions in $r s e A$ resulted in a 12-fold increase whereas deletion mutants exhibited a 25-fold induction of RpoE activity (De Las Penas et al., 1997; Missiakas et al., 1997). The large up-regulation upon the loss of $r s e A$ suggested that the major role of RseA is to act as a negative regulator of RpoE activity. Inactivation of $m u c A$ in $P$. aeruginosa resulted in a mucoid phenotype suggesting that MucA is a negative regulator of alginate production (Fyfe and Govan, 1980; Govan and Fyfe, 1978; Martin et al., 1993a; Mathee et al., 1999). Mapping and sequence analysis showed that mucA was located directly downstream of $\operatorname{algT/U}$ (Martin et al., 1993a).

Both RseA and MucA are localized to the inner membrane with the $\mathrm{N}$-terminus in the cytoplasm and C-terminus in the periplasm (De Las Penas et al., 1997; Lewenza et al., 2005; Mathee et al., 1997; Missiakas et al., 1997). Membrane preparations of RseAoverproducing bacteria showed RpoE co-eluted with RseA (Missiakas et al., 1997).

Pull-down experiments using RpoE-specific antibodies showed RseA and RpoE co-immunoprecipitating at a molar ratio close to 1:1 (Missiakas et al., 1997). In case of $P$. aeruginosa, co-expression of MucA and AlgT/U followed by cross-linking (Boucher et al., 1996) suggested MucA and AlgT/U interact directly. The interaction of MucA and AlgT/U 
is was supported by the co-fractionation of MucA and $\mathrm{AlgT} / \mathrm{U}$ in glycerol sedimentation experiments (Xie et al., 1996).

\section{E. coli RseB and $P$. aeruginosa MucB - Anti-anti sigma factors}

RseB and MucB share only 27.6\% homology (Table 1.3), however, they are functional equivalents that interact with the anti-sigma factors and subsequently affect alternative sigma factor activity. Deletion of $r s e B$ in $E$. coli resulted in an increase in $r p o E$ expression; whereas loss of $m u c B$ resulted in alginate production (Goldberg et al., 1993; Martin et al., 1993a). These findings clearly argued that RseB and MucB are negative regulators of $\mathrm{RpoE}$ and $\mathrm{AlgT} / \mathrm{U}$, respectively. Both RseB and MucB were localized to the periplasm (Boucher et al., 1996; Mathee et al., 1997; Missiakas et al., 1997). Direct interaction between RseB and MucB with their cognate anti-sigma factors RseA and MucA, respectively, has been demonstrate (Missiakas et al., 1997; Rowen and Deretic, 2000). Specifically, RseB and MucB were found to interact with the periplasmic Cterminal domain of their cognate anti-sigma factors (Cezairliyan and Sauer, 2007; Wood and Ohman, 2009). RseB is present as a dimer that protects RseA from cleavage by DegS (Wollman and Zeth, 2007; Cezairliyan and Sauer, 2007). In the case of MucB, it exists in both monomeric and dimeric forms, however; only the dimer binds to MucA (Cezairliyan and Sauer, 2009). The RseA-RseB interaction appears to stabilize RseA and strengthens RseA's bond with RpoE (Ades et al., 1999; Collinet et al., 2000). 
Table 1.2. List of proteins involved in RpoE regulation

\begin{tabular}{|c|c|c|c|c|c|c|c|c|}
\hline \multicolumn{4}{|c|}{ P. aeruginosa } & \multicolumn{4}{|l|}{ E. coli } & \multirow{2}{*}{$\begin{array}{c}\text { Product } \\
\text { Name }\end{array}$} \\
\hline $\begin{array}{c}\text { Locus } \\
\text { Tag }\end{array}$ & Protein & $\begin{array}{c}\text { Alternative } \\
\text { Name }\end{array}$ & $\begin{array}{l}\text { Size } \\
\text { (aa) }\end{array}$ & $\begin{array}{c}\text { Locus } \\
\text { Tag }\end{array}$ & Protein & $\begin{array}{c}\text { Alternative } \\
\text { Name }\end{array}$ & $\begin{array}{l}\text { Size } \\
\text { (aa) }\end{array}$ & \\
\hline PA0762 & $\mathrm{A} \lg \mathrm{T} / \mathrm{U}$ & $\mathrm{RpoE} / \sigma^{22}$ & 193 & ECK2571 & RpoE & $\sigma^{24} / \sigma^{\mathrm{E}} / \mathrm{SigE}$ & 191 & $\begin{array}{l}\text { Sigma } \\
\text { factor }\end{array}$ \\
\hline PA0763 & MucA & & 194 & ECK2570 & RseA & MclA/YfiJ & 216 & $\begin{array}{l}\text { Anti-sigma } \\
\text { factor }\end{array}$ \\
\hline PA0764 & MucB & $\mathrm{AlgN}$ & 316 & ECK1569 & RseB & $\mathrm{MclB}$ & 318 & $\begin{array}{l}\text { Negative } \\
\text { regulator }\end{array}$ \\
\hline PA0765 & MucC & $\mathrm{AlgM}$ & 151 & ECK2568 & RseC & $\mathrm{MclC}$ & 159 & \\
\hline PA0766 & MucD & $\mathrm{Alg} \mathrm{Y} / \mathrm{H} \operatorname{tr} \mathrm{A}$ & 474 & ECK0160 & DegP & $\mathrm{Htr} \mathrm{A} / \mathrm{Ptd} / \mathrm{Do}$ & 475 & $\begin{array}{l}\text { Serine } \\
\text { endoprotease }\end{array}$ \\
\hline PA4446 & $\mathrm{AlgW}$ & & 389 & ECK3224 & DegS & HhoB/HtrH & 355 & $\begin{array}{l}\text { Site } 1 \text { protease; } \\
\text { HtrA family }\end{array}$ \\
\hline PA3649 & MucP & & 450 & ECK0175 & RseP & YaeL/EcfE & 450 & $\begin{array}{l}\text { Site } 2 \text { protease; } \\
\text { Zn dependent }\end{array}$ \\
\hline PA4033 & MucE & & 89 & $\begin{array}{c}\text { No } \\
\text { homologs }\end{array}$ & & & & \\
\hline PA1802 & ClpX & ClpX & 426 & ECK0432 & ClpX & & 424 & $\begin{array}{l}\text { ATPase subunit } \\
\text { of the protease }\end{array}$ \\
\hline PA1801 & ClpP & Clp1 & 213 & \multirow{2}{*}{ ECK0431 } & \multirow{2}{*}{ ClpP } & \multirow{2}{*}{ LopP/WseA } & \multirow{2}{*}{201} & \multirow{2}{*}{$\begin{array}{l}\text { Proteolytic } \\
\text { subunit of the } \\
\text { protease }\end{array}$} \\
\hline PA3326 & Clp2 & & 201 & & & & & \\
\hline PA4427 & SspB & & 135 & ECK3217 & SspB & & 165 & $\begin{array}{l}\text { Stringent } \\
\text { starvation } \\
\text { protein } \\
\end{array}$ \\
\hline
\end{tabular}


Table 1.3. Comparison of $E$. coli and $P$. aeruginosa homologs involved in the anti-sigma factor (RseA and MucA, respectively) proteolysis

\begin{tabular}{|c|c|c|c|c|c|c|c|c|c|c|c|c|}
\hline & \multicolumn{11}{|c|}{ E. coli K12 } \\
\hline & & RpoE & RseA & RseB & RseC & DegP & DegS & RseP & ClpX & ClpP & SspA & SspB \\
\hline \multirow{12}{*}{  } & AlgT/U & 66.0 & 7.8 & 18.7 & 15.2 & 20.7 & 18.8 & 21.2 & 25.4 & 16.2 & 9.3 & 15.7 \\
\hline & MucA & 14.4 & 28.3 & 22.2 & 18.2 & 16.1 & 15.0 & 18.6 & 17.0 & 13.3 & 14.9 & 13.3 \\
\hline & MucB & 19.4 & 16.2 & 27.6 & 29.9 & 17.2 & 11.4 & 19.5 & 18.4 & 17.0 & 19.3 & 18.1 \\
\hline & MucC & 15.9 & 17.2 & 21.2 & 30.0 & 23.2 & 26.5 & 24.5 & 22.5 & 10.7 & 17.3 & 13.3 \\
\hline & MucD & 22.0 & 19.4 & 17.9 & 15.0 & 39.2 & 33.4 & 15.4 & 15.2 & 23.2 & 16.9 & 19.3 \\
\hline & AlgW & 17.4 & 17.6 & 15.8 & 23.9 & 36.8 & 46.3 & 17.2 & 14.3 & 20.3 & 18.4 & 18.7 \\
\hline & MucP & 17.3 & 17.6 & 19.8 & 23.2 & 10.2 & 16.7 & 46.9 & 11.9 & 17.9 & 23.5 & 22.4 \\
\hline & ClpX & 23.0 & 15.3 & 15.1 & 19.5 & 16.2 & 17.9 & 12.6 & 76.6 & 19.3 & 21.2 & 17.5 \\
\hline & ClpP & 12.0 & 11.3 & 16.1 & 18.9 & 18.8 & 17.4 & 19.7 & 17.4 & 69.6 & 13.2 & 14.5 \\
\hline & ClpP2 & 16.2 & 12.6 & 19.4 & 19.6 & 14.9 & 19.4 & 16.4 & 22.6 & 41.5 & 14.2 & 15.7 \\
\hline & SspA & 13.2 & 11.5 & 20.0 & 20.1 & 18.6 & 17.6 & 19.5 & 21.2 & 12.6 & 53.9 & 15.2 \\
\hline & SspB & 16.3 & 19.2 & 20.0 & 18.0 & 21.5 & 20.8 & 22.2 & 18.5 & 16.3 & 20.0 & 53.3 \\
\hline
\end{tabular}

The numbers refer to percentage homology as determined using ClustalW (Table 1.3). The shaded boxes compare the functional homologs. The protein sequences of $E$. coli and $P$. aeruginosa were retrieved from ecogene.com and pseudomonas.com. 
Coaggregation of RseB with misfolded proteins suggests that it may act as a stress sensor, which may allow RseB to release from RseA to initiate the RIP cascade leading to RpoE-dependent activity (Collinet et al., 2000).

\section{E. coli RseC and P. aeruginosa MucC}

Though both $E$. coli rseC and $P$. aeruginosa mucC are the third gene in their respective rpoE operons, they share only $30 \%$ homology. The role of $\mathrm{RseC}$ and $\mathrm{MucC}$ is ambiguous and little is currently known about the exact cellular function of these members of the rpoE and algT/U operons (Boucher et al., 1997a; De Las Penas et al., 1997; Franklin and Ohman, 1996; Missiakas et al., 1997).

\section{E. coli DegP and P. aeruginosa MucD}

E. coli degradation protein $\underline{\mathrm{P}}(\mathrm{DegP})$ that is required for growing the bacterium above $42{ }^{\circ} \mathrm{C}$ and P. aeruginosa Mucoid D (MucD) share 39\% homology (Table 1.3)(Gottesman and Zipser, 1978; Strauch et al., 1989b). The presence of $m u c D$ in the algT/U operon suggests that MucD should play a role in the post-translational regulation of $A \lg T / \mathrm{U}$. In the case of $E$. coli, $\operatorname{deg} P$ is not present in the rpoE operon (Strauch and Beckwith, 1988). Expression of both $\operatorname{deg} P$ and $m u c D$ are under the control of RpoE and AlgT/U, respectively (Boucher et al., 1996; Dartigalongue et al., 2001; Erickson and Gross, 1989; Franklin and Ohman, 1996; Lipinska et al., 1988; Wang and Kaguni, 1989).

Though mutations in $\operatorname{deg} P$ had no effect on E. coli rpoE transcription, DegP is required for its growth at elevated temperatures (Lipinska et al., 1989; Lipinska et al., 1990). Both null and point mutations of mucD in non-mucoid PAO1 resulted in a mucoid phenotype conversion (Boucher et al., 1996; Wood et al., 2006). Alkaline-phosphatase 
fusions of DegP and MucD showed them to be localized to the periplasm (Lewenza et al., 2005; Strauch et al., 1989a). The major role of both DegP and MucD is to degrade misfolded proteins from the periplasm (Strauch and Beckwith, 1988; Wood and Ohman, 2009) that may indirectly regulate RseB and MucB allowing it to re-interact with their cognate anti-sigma factors.

\section{E. coli DegS and P. aeruginosa AlgW}

The degradation protein $\mathrm{S}(\mathrm{DegS})$ and Alginate $\mathrm{W}(\mathrm{AlgW})$ share substantial homology (Table 1.3). They are also members of the HtrA family of serine proteases (Pallen and Wren, 1997). DegS and AlgW are also known as Site-1 proteases (S1P), which cleave anti-sigma factors on their periplasmic sides (Dalbey et al., 2012; Qiu et al., 2007).

DegS is an inner membrane protein with a large periplasmic domain (Alba et al., 2002); (Waller and Sauer, 1996). Based on sequence homology, AlgW is also presumed to be an inner membrane protein with a single transmembrane domain. DegS initiates the first cleavage of RseA leading to an eventual release of RpoE to initiate transcription (Ades $e t$ al., 1999; Alba et al., 2002). DegS cleaves between amino acids Val148 and Ser149 in the transmembrane domain of RseA adjacent to the periplasmic side (Walsh et al., 2003). AlgW primarily cleaves MucA between amino acids Ala136 and Gly137 (Cezairliyan and Sauer, 2009). DegS binding of the C-terminal -YXF motif of misfolded OMPs activates it to cleave RseA (Walsh et al., 2003). But, in P. aeruginosa, mисE that encodes an outer

membrane associated small protein bind with a specific C-terminal -WVF motif and initiates RIP cascade (Qiu et al., 2007). 


\section{E. coli RseP and P. aeruginosa MucP}

Both RseP and MucP are Site-2 Proteases (S2P) which are membrane-bound zinc metalloproteinases involved in the proteolytic activation of regulatory factors for sterol biosynthesis and for stress responses (Kanehara et al., 2001; Kinch et al., 2006; Qiu et al., 2007). RseP and MucP were both identified based on sequence homology to other site-2proteases (Kanehara et al., 2001; Qiu et al., 2007). RseP is essential in E. coli as depletion causes a rapid loss of viability, cell elongation, and growth cessation (Kanehara et al., 2001).

RseP is an inner membrane protein with four transmembrane domains with both the $\mathrm{N}$ - and $\mathrm{C}$ - termini lying in the periplasm (Kanehara et al., 2001). RseP also contains two periplasmic PDZ domains involved in its regulation (Inaba et al., 2008); (Kanehara et al., 2003). Our analysis using TMHMM shows MucP possessing a similar domain structure to RseP. Cleavage of RseA by RseP was confirmed using in vitro assays (Akiyama et al., 2004). The regulated proteolysis of RseA requires a functional PDZ domain of RseP (Kanehara et al., 2003). Indirect genetic evidence for MucP cleavage of MucA leading to AlgT/U expression has been shown using mutant strains (Damron et al., 2011; Qiu et al., 2007). No direct interaction with MucP and MucA has been demonstrated.

\section{E. coli and P. aeruginosa ClpXP}

After RseP-cleavage of RseA, a small fragment of RseA remains attached to RpoE (De Las Penas et al., 1997; Missiakas et al., 1997). The removal of this RseA cytoplasmic fragment requires SspB and ClpXP (Flynn et al., 2004). SspB was found to bind to both 
the amino-terminus of RseA as well as ClpX thereby bringing the fragment close enough to the ClpXP protein complex to allow for RseA fragment degradation (Flynn et al., 2004).

In P. aeruginosa, there are two ClpP homologs, ClpP (PA1801) and ClpP2 (PA3326). Mutagenesis of $c l p X, c l p P$, and $\operatorname{clp} P 2$ individually resulted in the loss of a mucoid phenotype (Qiu et al., 2008a). P. aeruginosa also harbors the sspAB (PA4426PA4427) operon (Yin et al., 2013). Unlike E. coli, SspA appears to play a role in alginate production (Yin et al., 2013). A direct interaction between the MucA fragment and ClpXP or SspA has yet to be demonstrated.

\section{RIP Models for E. coli and P. aeruginosa}

Many questions remain unanswered. However, on the basis of the above findings, the following model is proposed for E. coli (Figure 1.7A). and P. aeruginosa (Figure 1.7B).

\section{E. coli RIP cascade}

Misfolded OMP peptides with exposed C-terminal -YQF motifs bind to the PDZ domain of DegS allowing cleavage of RseA at the periplasm. It is also presumed that misfolded peptides compete for binding with RseB from RseA allowing cleavage of RseA

by DegS. Alternatively, it has been postulated that RseB hinders RseP-recognition of RseA. Following cleavage by DegS, RseP cleaves RseA at the cytoplasm releasing RpoE with an $\mathrm{N}$-terminal fragment of RseA. This $\mathrm{N}$-terminal fragment of RseA remains attached until removed by SspB-ClpXP releasing the free RpoE to initiate transcription of the RpoE regulon. 


\section{P. aeruginosa RIP cascade}

Similar to the E. coli system, MucA is sequentially degraded by $\mathrm{AlgW}$, and MucP. The C-terminal -WVF motif of MucE has been shown to prime the $\mathrm{AlgW}$ protease to initiate the cleavage of MucA. The direct cleavage of MucA by MucP, or interaction of ClpXP or SspA/B has not been demonstrated but inferred from genetic data and by comparison to the E. coli system. In $P$. aeruginosa $\mathrm{SspB}$ appears to play a role in this pathway. Free $\mathrm{Alg} \mathrm{T} / \mathrm{U}$ activates all the genes in the alg regulon, leading to alginate production.

\section{Inducers of alginate production}

\section{Chemical}

\section{Ammonium metavanadate}

Pseudomonas isolation agar supplemented with ammonium metavanadate $[0.27 \mathrm{mM}]$ induces alginate production in $P$. aeruginosa (Damron et al., 2011). Growing $P$. aeruginosa in ammonium metavanadate increases the expression of periplasmic stress chaperones MucD and well as cytoplasmic chaperones GroEL [PA4385], DnaK [PA4761], GrpE [PA4762]; and outer membrane proteins [OMP] OprF [PA1777], and OprO [PA3280] but exactly how the chemical induces the mucoid phenotype is not clear yet (Damron et al., 2011). The mucoid $P$. aeruginosa grown on pseudomonas isolation agar (Imperi et al.) supplemented with ammonium metavanadate when streaked back to PIA, the mucoid $P$. aeruginosa turned non-mucoid suggesting ammonium metavanadate based mucoid phenotype is not due to the genetic change in the bacterium (Damron et al., 2011). 





Figure 1.7. Proposed model for the anti-sigma factor proteolysis in $E$. coli (A) and $P$. aeruginosa (B). The model is based on currently available data. (A) The steps for E. coli are outlined as follows: (i) -YXF C-terminal motif exposed in misfolded OMPs binds to the PDZ domain of DegS activating its proteolytic domain. DegP may degrade misfolded OMP proteins prior to their interaction with DegS, perhaps preventing DegS activation; Based on $P$. aeruginosa system, RseB dissociates to allow site-1 proteolysis of RseA at its periplasmic side by DegS; (iii) The removal of C-terminal RseA allows for site-2 proteolysis at its cytoplasmic side by RseP. It has been postulated that RseP access to RseA can also be prevented by RseB; (iv) RseP activity releases RpoE with a fragment of RseA bound to it; (v) SspB binds to and brings the RseA fragment to the ClpXP proteasome; (vi) ClpXP degradation of the RseA fragment releases free RpoE; (vii) Free RpoE directs RNA polymerase that is made of five subunits $\left(\alpha_{2} \beta \beta^{\prime} \omega\right)$ to initiate transcription of the RpoE regulon. (B) The model is based on the available data and is, in part, inferred from the $E$. coli system. The steps involved are as follows: (i) -WVF motif on MucE binds to the AlgW PDZ domain activating its proteolytic activity. MucD is hypothesized to inhibit the interaction of MucE with $\mathrm{AlgW}$, as well as binding misfolded proteins; (ii) Activated $\mathrm{AlgW}$ cleaves the periplasmic domain of MucA releasing $\mathrm{MucB}$ along with a $\mathrm{C}$-terminal fragment of MucA; (iii) MucP induces site-2 proteolysis of MucA at the cytoplasmic side releasing AlgT/U with a small N-terminal fragment of MucA attached; (iv) Based on the E. coli system, SspA is proposed to be the adaptor molecule that brings AlgT/U with the MucA fragment to ClpXP; (V) The MucA fragment is removed from AlgT/U by the ClpXP proteasome. The role of ClpP2 homolog is not clear; (vii) Free AlgT/U directs RNA polymerase to initiate transcription of the AlgT/U regulon.

\section{Imipenem}

Growing $P$. aeruginosa in biofilms upon exposure to the antibiotic imipenem [1.0 $\mu \mathrm{g} / \mathrm{ml}]$ for 24 hours cause the appearance of a mucoid phenotype (Bagge et al., 2004). However, sub-culturing the mucoid $P$. aeruginosa from the imipenem-exposed biofilm on nonselective LB agar plates did not show the mucoid phenotype suggesting that the observed mucoidy was unstable (Bagge et al., 2004).

\section{Ethanol}

Mucoid $P$. aeruginosa were obtained when the bacterium was grown for 6 days at room temperature $\left(22^{\circ} \mathrm{C}\right)$ on (Yeast extract, tryptone, and glucose) YTG medium 
supplemented with 3\% - 5\% ethanol (Devault et al., 1990). The frequency of mucoid variant formation was one colony per 106 cells but the mucoid phenotype lasted for 14 days when grown in the same medium without ethanol before it reverted back to nonmucoid phenotype (Devault et al., 1990).

\section{Phage}

Phage was isolated from the supernatant of phage-induced-mucoid variants of $P$. aeruginosa and applied to the non-mucoid strain of $P$. aeruginosa, which caused the nonmucoid strain to turn mucoid suggesting phage can also induce mucoidy in $P$. aeruginosa (Martin, 1973).

\section{Estradiol}

It has been shown in both human and mouse model that estradiol enhances the alginate production in $P$. aeruginosa may be responsible for a survival disadvantage of female patients with CF (Chotirmall et al., 2012).

\section{Mucoid phenotype and antibiotic resistance of $P$. aeruginosa}

It has been proposed that alginate production contributes to antibiotic resistance in $P$. aeruginosa, but several results show that there is no correlation between mucoidy and antibiotic resistance. There is no significant effect of the antibiotics tobramycin and tigecycline to the wild-type PAO1 and mucoid P. aeruginosa (Oglesby-Sherrouse et al., 2014). 


\section{Stability}

It is commonly known that mucoid clinical $P$. aeruginosa reverts to the non-mucoid phenotype (Govan, 1975; Zierdt and Schmidt, 1964). For the clinical P. aeruginosa FRD strain, incubation of the strain in L-broth at $40{ }^{\circ} \mathrm{C}$ without aeration results in mucoid to non-mucoid conversion at the rate of $50 \%$ within $24-48$ hours (Ohman and Chakrabarty, 1981). Mucoid to non-mucoid conversion is faster when the bacteria are grown without aeration in the nutrient medium compared to the bacteria grown with aeration in deoxycholate citrate agar (Govan, 1975). In case of the PAO1-derivative PDO300, the rate of mucoid to non-mucoid conversion was found to be $90 \%$ within 48 hours (Sautter et al., 2012). The mucoid to non-mucoid conversion has been mapped to mutations in the algT/U operon, algO, algW, and mucP (Boucher et al., 1996; Qiu et al., 2007; Reiling et al., 2005; Sautter et al., 2012; Schurr et al., 1994). The switch from non-mucoid to a mucoid phenotype is associated with a poor prognosis for CF patients (Pillarisetti et al.). The alginate over-production enables $P$. aeruginosa to cause persistent infections in the airways of $\mathrm{CF}$ lungs that results in decreased lung function, increases the risk of respiratory failure, and ultimately the demise of the CF patients. The change from non-mucoid to mucoid phenotype is the result of the mutation of anti-sigma factor MucA resulting in the loss of regulation of the alternate sigma factor $\mathrm{Alg} \mathrm{U}$. The mutation allows $\mathrm{Alg} \mathrm{U}$ to become free to interact with core RNA polymerase and direct transcription of the alginate biosynthetic genes located in the $\operatorname{alg} D$ operon, in addition to its own operon $\operatorname{alg} U-$-тисA-тисB-тисCmисD. The alternate sigma factor AlgU belongs to the extra-cytoplasmic function (ECF) family. $\mathrm{AlgU}$ (also called $\mathrm{AlgT}$ ) is $22 \mathrm{kDa}$ protein that shares $66 \%$ identity with the E. coli 
sigma factor RpoE. In case of $\mathrm{Alg} \mathrm{T} / \mathrm{U}$ the mutation was mapped to codon 18 , where a GAC in $\left(\mathrm{Alg}^{+}\right)$strains was changed to GGC that encodes glycine (De Las Penas et al., 1997). Another change was at codon 29, where TAC [Tyr] was changed to TGC [Cys]. Both of mutation in codon 18 or codon 29 makes AlgT/U unable to direct the transcription resulting in a non-mucoid phenotype of $P$. aeruginosa.

\section{Strategy to map suppressor of alginate production mutations}

\section{Cosmid-based technique}

A minimum tiling cosmid library that covers $93.7 \%$ of the $P$. aeruginosa genome with 334 colonies is commonly used to map mutation in the genome (Huang et al., 2000).

\section{Localized mutagenesis}

The localized mutagenesis technique is similar to the one used to obtain temperature-sensitive mutant Salmonella typhimurium where transducing phage is in vitro mutagenized prior to transduction. To find the novel gene involved in acetylation of alginate, small fragment of bacterial DNA encoding the alginate biosynthetic operon was placed in a cosmid. Lambda lysate was prepared on E. coli HB101 to package these cosmids into phage particles. The packaged cosmids were mutagenized in vitro by treatment with hydroxylamine. Hydroxylamine is a highly specific mutagen that reacts with cytosine and causes the specific replacement of nucleotide G by an A. Hydroxylamine is a highly specific mutagen that reacts with cytosine and causes the specific replacement of nucleotide $\mathrm{G}$ by an $\mathrm{A}$. Then the mutagenized cosmid is packed in the phage and transduced into E. coli. 
Then the mutagenized cosmid is packed in the phage and transduced into E. coli. Then triparental mating is done to transfer the mutagenized cosmid from E. coli to $P$. aeruginosa and finally, $P$. aeruginosa carrying the mutagenized cosmid is screened for the particular phenotype (Franklin and Ohman, 1996).

\section{Sequencing-based technique}

In whole genome sequencing-based approach purified DNA from the suppressor strain can be used for sequencing using high throughput sequencing platform. Several sequencing platforms such as Illumina Hiseq200 can be used.

\section{Purpose of my study}

Our lab isolated nonmucoid revertants termed as a suppressor of alginate production strains (sap) derived from the constitutively mucoid $\left(\mathrm{Alg}^{+}\right)$PDO300. One of the previously uncharacterized sap mutants, sap27 (non- mucoid), was used in the present study. Complementation of the sap27 by a cosmid pMO012217 from the $P$. aeruginosa cosmid library restored the mucoid phenotype of sap27 suggesting the at least one the genes in the cosmid is required for the mucoid phenotype. Preliminary transposon mutagenesis suggests a three-gene operon (lptD-surA-pdxA; PA0595-PA0594-PA0593), is involved in alginate overproduction. To map the specific gene responsible for this complementation in the cosmid pMO02217, and to understand its role in alginate production/regulation, the project has following three aims:

\section{Specific Aim 1: To map the genes on pMO012217 involved in alginate}

production. The working hypothesis is that one of the 18 open reading frames (ORF) present in the cosmid is responsible for restoring the mucoid phenotype in sap27. The 
preliminary result suggests a three-gene operon (lptD-surA-pdxA), is involved in alginate overproduction. The genes were individually expressed in sap 27 to identify the gene involved in alginate production. In addition, the gene in sap27 was sequenced. Subsequently, the role of the gene in alginate production was determined.

Specific Aim 2: To determine the mechanism of action of LptD. The working hypothesis is that LptD bypasses the proteins that are involved in alginate production in sap27. To test this, $\mathrm{LptD}$ was overexpressed in the strains whose previously known alginate related gene was deleted and scored for the revertant mutant to understand the gene hierarchy of LptD in RIP cascade.

Specific Aim 3: To identify the role of PLP in virulence of $P$. aeruginosa. Preliminary data show an exogenous addition of pyridoxal 5' -phosphate (PLP) can affect

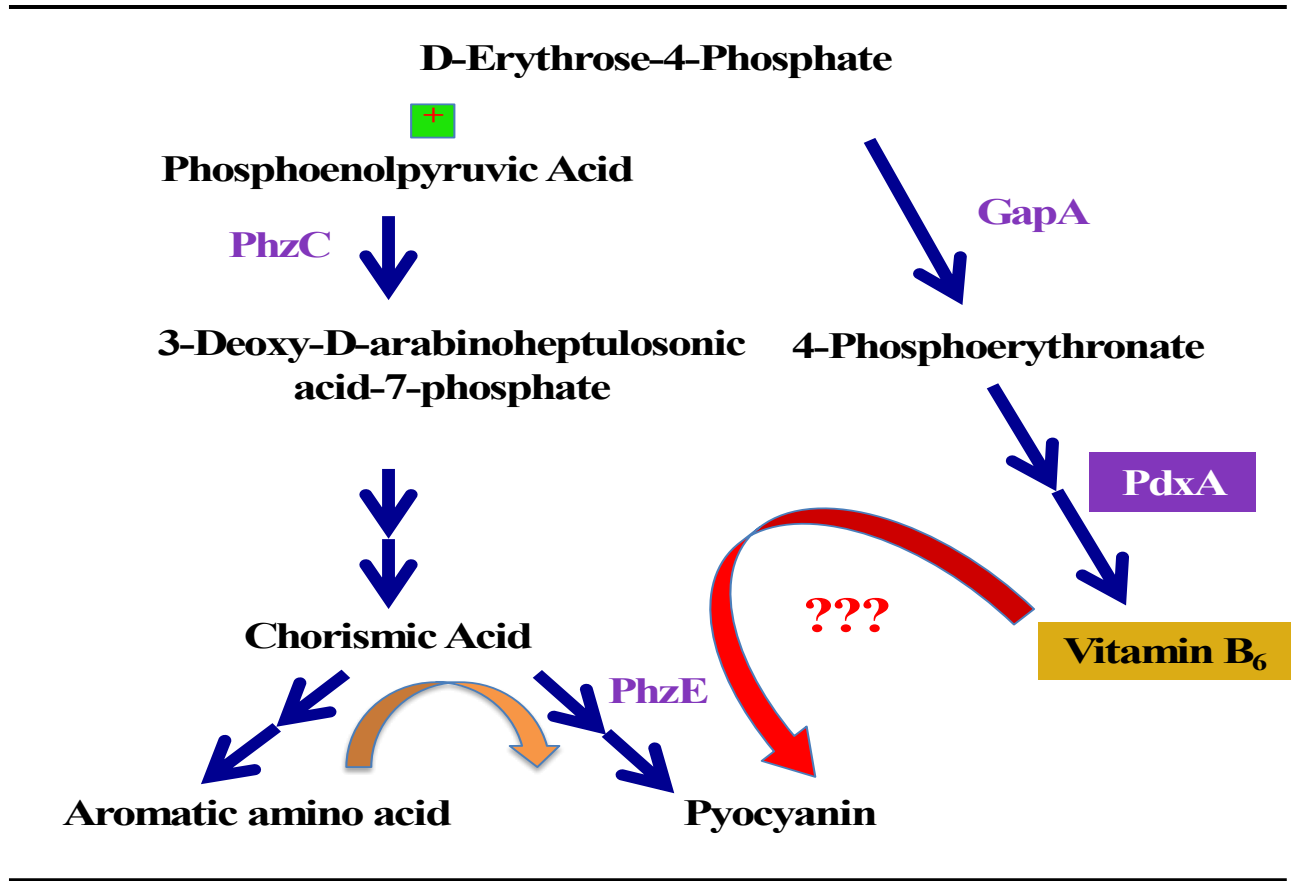

Figure 1.8. Pyocyanin production pathway in $P$. aeruginosa. Erythose-4-phosphate and phosphoenol pyruvate are precursors for synthesis of both vitamin $\mathrm{B}_{6}$ and shikimiate. Then shikimate is converted into chorismic acid that eventually converted into pyocyanin. Culturing $P$. aruginosa in a media with vitamin $\mathrm{B}_{6}$ or in presence of aromatic amino acid directs the pathway to pyocyanin production. 
pyocyanin production. To understand the pathway for pyocyanin production we used quantitative PCR of the genes shown in purple color (Figure 1.8). 


\section{CHAPTER 2}

Outer membrane protein LptD (PA0595) plays a role in the regulation of alginate synthesis in Pseudomonas aeruginosa

This chapter has been published:

Sundar Pandey, Camila Delgado, Hansi Kumari, Laura Florez, and Kalai Mathee DOI: $10.1099 / \mathrm{jmm} .0 .000752$

Journal of Medical Microbiology 


\begin{abstract}
The emergence of increased alginate producing mucoid strains is linked to a poor prognosis in people with cystic fibrosis. The mucoid phenotype is very unstable outside of the CF lung. In this study, a set of nonmucoid revertant strains was used to identify novel genes involved in alginate production. Complementation analysis of these mutants using a minimal tilling path cosmid library identified a cosmid pMO012217 that restored the mucoid phenotype of sap27. The cosmid contains 18 open reading frames. Transposon mutagenesis of the cosmid mapped the mutation to LptD (PA0595) encoding a Lipopolysaccharide transport protein D (LptD), a protein associated with lipopolysaccharide transport to the outer membrane of the bacterium. We established LptD


mucoid phenotype of sap27. We took the advantage of PAO1, and its isogenic mucA22 mutant PDO300; and $\operatorname{algO}$, and $\operatorname{alg} W$ mutant of both of these mutants as well of the plasmids harboring $\operatorname{alg} O$ and $\operatorname{alg} W$ to identify the pathways that restored the mucoid phenotype of the bacterium due to LptD.
\end{abstract}

\title{
Introduction
}

Pseudomonas aeruginosa, a Gram-negative, ubiquitous bacterium is pathogenic to plants, animals, and humans (Bayes et al., 2016; McAvoy et al., 1989; Rahme et al., 1995). The most severe $P$. aeruginosa infections in humans include endocarditis, malignant otitis externa, septicemia, endophthalmitis, eye keratitis, pneumonia, and meningitis (Bodey et al., 1983). Annually, there are 51,000 P. aeruginosa infections of which 6,700 are multidrug-resistant isolates causing 440 deaths in the USA (2015). The Centers for Disease 
Control and Prevention has ranked $P$. aeruginosa as a pathogen of serious threat level (CDC, 2015).

P. aeruginosa is the leading cause of morbidity and mortality in patients with $\mathrm{CF}$ (2016). Colonization of the lungs of CF patients starts a few years after birth and remains for life (Hoiby et al., 1977) An early acute infection transitions to a chronic phase when isolated $P$. aeruginosa colonies exhibit a mucoid phenotype due to constitutive production of alginate $\left(\mathrm{Alg}^{+}\right)$(Doggett et al., 1971; Hoiby et al., 1977). Though alginate-producing $P$. aeruginosa is primarily associated with CF individuals, occasionally it has been isolated from patients with bronchiectasis, urinary tract, and middle-ear infections (McAvoy et al., 1989). Mucoid P. aeruginosa strains have also been isolated from wastewater systems, and the guttural pouch of an equine with chronic mucopurulent nasal discharge (Govan et al., 1992; Grobe et al., 1995).

The presence of alginate-producing strains in the lungs of CF patients is associated with poor prognosis (Hoiby, 1975; Pedersen et al., 1992). Alginate protects $P$. aeruginosa from phagocytosis, antibiotics, oxygen radicals, and the host immune response (Govan and Fyfe, 1978; Hodges and Gordon, 1991; Kulczycki et al., 1978; Mathee et al., 1999; Oliver and Weir, 1985; Schwarzmann and Boring, 1971). The importance of alginate in the virulence of $P$. aeruginosa has also been demonstrated in mouse models (Boucher et al., 1997a; Bragonzi et al., 2006; Pier et al., 2001; Song et al., 2003; Yu et al., 1998). Compared to wild-type $P$. aeruginosa, an alginate-overproducing strain causes aggressive polymorphonuclear (PMN) leukocyte infiltration (similar to human infection) and causes inefficient pulmonary clearance (Song et al., 2003). A protracted lung infection has the 
potential to spread to other organs, such as the spleen has observed in the mouse model (Song et al., 2003). These properties suggest that alginate is an important virulence factor.

Exposing the bacteria to stress can induce alginate production. Mucoid conversion of $P$. aeruginosa in vitro can be triggered by growth in high osmolarity media, with limited nutrients (like nitrogen, phosphate, and carbon), static culture in acetamide broth, continued culture in the presence of antibiotics, Pseudomonas Isolation Agar supplemented with ammonium metavanadate, ethanol dehydration and extended exposure to oxygen radicals such as hydrogen peroxide (Damron et al., 2011; Devault et al., 1990; Evans and Linker, 1973; Govan and Fyfe, 1978; Mathee et al., 1999; Speert et al., 1990; Terry et al., 1991). Alginate production can also be stimulated by anaerobiosis (Bragonzi et al., 2006; Hassett, 1996; Worlitzsch et al., 2002). Though it is difficult to ascertain every factor involved, it appears that in $\mathrm{CF}$ patients, continuous exposure to antibiotics and PMNderived hydrogen peroxide may be the major contributors of mucoid conversion in $P$. aeruginosa (Mathee et al., 1999).

Alginate production is tightly controlled by a myriad of regulatory factors. AlgR, AmrZ (previously called as $\mathrm{AlgZ}$ ), $\mathrm{AlgB}$, and $\mathrm{AlgT} / \mathrm{U}$ regulate transcription of the algD operon (Baynham and Wozniak, 1996; Goldberg et al., 1993; Kato and Chakrabarty, 1991; Leech et al., 2008; Mohr et al., 1992; Worlitzsch et al., 2002; Wozniak and Ohman, 1991). The $\mathrm{AlgR}$ and $\mathrm{AlgB}$ proteins are response regulators of different two-component systems, FimS-AlgR, and KinB-AlgB that are essential for high levels of alginate synthesis (Goldberg and Dahnke, 1992; Goldberg and Ohman, 1987; Ma et al., 1997a; Whitchurch et al., 1996; Wozniak and Ohman, 1991; Yu et al., 1997). However, the conserved phosphorylation domains of both $\mathrm{AlgB}$ and $\mathrm{AlgR}$ are not required for alginate production 
(Damron et al., 2009; Ma et al., 1998; Worlitzsch et al., 2002). Phosphorylated AlgR is not needed for alginate production but plays a role in twitching motility (Whitchurch et al., 1996). Another protein that increases alginate production is AmrZ, a ribbon-helix-helix DNA binding protein (Baynham and Wozniak, 1996). The membrane-associated cyclicdimeric-GMP synthesizing protein MucR is also required for the synthesis of alginate in P. aeruginosa (Hay et al., 2009).

The master regulator of alginate synthesis is $\mathrm{AlgT} / \mathrm{U}\left(\sigma^{22}\right)$, which belongs to the family of the extracytoplasmic sigma factors $(\mathrm{ECF})$ and has high homology to $\operatorname{SigE}\left(\sigma^{\mathrm{E}}\right)$ found in E. coli, Streptomyces coelicolor, Bacillus subtilis and Salmonella Typhimurium (Devries and Ohman, 1994a; Hershberger et al., 1995; Lonetto et al., 1992; Martin et al., 1993; Worlitzsch et al., 2002; Wozniak and Ohman, 1994). Expression of algT/U is autoregulated, and $\mathrm{AlgT} / \mathrm{U}$, in turn, regulates expression of the $\operatorname{alg} D, \operatorname{alg} B, \operatorname{alg} R, \operatorname{amr} Z$ and mucE operons (Baynham and Wozniak, 1996; Devries and Ohman, 1994b; Wozniak and Ohman, 1994; Yin et al., 2013). The algT/U gene is a part of the algT/U-mисA-тисBтисC-тисD operon that plays an essential role in converting nonmucoid $P$. aeruginosa into its mucoid phenotype (Devries and Ohman, 1994a; Goldberg et al., 1993; Martin et al., 1993). MucA is an inner membrane protein, and its $\mathrm{N}$-terminal domain in the cytoplasm sequesters AlgT/U and prevents its activity (Mathee et al., 1997; Xie et al., 1996). Most mucoid CF isolates acquire mutations in mисA, and $84 \%$ of these harbor a common allele known as mucA22 that has a deletion of one nucleotide in a stretch of five guanine nucleotides located at position $426-430$ of the open reading frame (ORF) resulting in premature translation termination (Boucher et al., 1997b; Bragonzi et al., 2006; Martin et al., 1993). MucA truncation leads to the constitutive production of alginate. In addition, 
inactivation of $m и с B$ and $m u c D$ also leads to constitutive alginate production (Boucher $e t$ al., 1996; Goldberg et al., 1993; Martin et al., 1993; Schurr et al., 1996).

In $P$. aeruginosa, MucE accumulates in response to cell wall stress (Step 1, Figure. 1.7(B)) then the site-1 RIP serine-protease $\mathrm{AlgW}$ is activated to clip the C-terminus of MucA between amino acids Ala136 - Gly137 in the periplasm (Step 2, Figure. 1.7(B)) (Cezairliyan and Sauer, 2009; Qiu et al., 2007). Then, the site-2 zinc-metalloprotease MucP cleaves MucA within the inner membrane (Step 3, Fig. 1.7(B)) but the exact cleavage site remains to be elucidated (Damron et al., 2011; Wood and Ohman, 2009). The MucA fragment tethered to $\mathrm{AlgT} / \mathrm{U}$ is further processed by a cytoplasmic protease protein complex ClpXP with the aid of the SspA protein, finally releasing the sigma factor AlgT/U (Qiu et al., 2008a; Yin et al., 2013). The MucA C-terminus that lies on the periplasmic side is presumed to bind MucB (Cezairliyan and Sauer, 2009; Mathee et al., 1997). It has been shown in vitro that the presence of MucB in the reaction mixture prevents the cleavage of MucA by the periplasmic protease AlgW (Cezairliyan and Sauer, 2009). MucD is a chaperone/protease that controls periplasmic protein quality and can trigger the degradation of MucA upon detection of abnormal protein accumulation (Damron and $\mathrm{Yu}$, 2011; Wood and Ohman, 2006). In the mucD mutants, an alternate RIP pathway appears to be activated in which MucP directly cleaves MucA without pre-cleavage by $\mathrm{AlgW}$ (Damron and Yu, 2011; Qiu et al., 2007). The role of MucC is currently controversial: reportedly being a positive and a negative regulator of alginate production (Boucher et al., 1997a; Ohman, 1996). AlgO is a serine periplasmic protease that positively affects alginate production in P. aeruginosa; however, its precise role in the RIP cascade remains to be elucidated (Reiling et al., 2005; Sautter et al., 2012). AlgO, AlgW, and MucD contain PDZ 
(PSD-95/Discs-large/ZO1) domains that are involved in the protein-protein interaction (Ponting, 1997). In the wild-type background, the release of AlgT/U requires the sequential degradation of MucA through a process known as regulated intramembrane proteolysis (RIP) (Figure. 1.7), a process that is conserved from bacteria to humans (Ruiz et al., 2008; Wood and Ohman, 2009).

Besides mutations in biosynthetic and regulatory genes, alginate production in $P$. aeruginosa can be suppressed by various means that includes increasing the copy number of genes such as $r p o D$, prolonged growth in the presence of sulfate ions or without aeration (Min et al., 2014; Ohman and Chakrabarty, 1981; Yin et al., 2013). The reversion phenotype has been exploited to study the regulation and to find novel genes associated with alginate production (Devries and Ohman, 1994b; Min et al., 2014; Sautter et al., 2012). The reversion from mucoid to nonmucoid, when grown without aeration, was mainly mapped to mutations in $\operatorname{alg} T / U$ and $\operatorname{algO}$ (Devries and Ohman, 1994a; Sautter $e t$ al., 2012). In this study, the reversion phenomenon was further exploited to map novel genes using a genetically well-defined constitutively mucoid strain, PDO300, with a тисA22 allele derived from the prototypic nonmucoid PAO1 strain to isolate nonmucoid variants that are termed suppressor of $\boldsymbol{a l g i n a t e}$ production (sap) mutants (Mathee $e t$ al., 1999; Sautter et al., 2012). One of the previously uncharacterized sap mutants, sap27, was used in this study. Complementation and mutational analysis led to the identification of lptD (PA0595), encoding an outer membrane protein as responsible for restoring the mucoid phenotype. However, sap27 had no mutation in $\operatorname{lptD}$, but in algO. This study will explore the role of $\mathrm{LptD}$ and $\mathrm{AlgO}$ in alginate production. 


\section{METHODS}

\section{Bacterial strains, plasmids, media and primers}

The E. coli and P. aeruginosa strains and plasmids used are listed in Table 2.1. E. coli was grown in Luria-Bertani (LB) medium supplemented with chloramphenicol (Cm, $\left.10 \mu \mathrm{g} \mathrm{ml}^{-1}\right)$, gentamicin $\left(\mathrm{Gm}, 15 \mu \mathrm{g} \mathrm{ml}^{-1}\right)$, and kanamycin $\left(\mathrm{Km}, 25 \mu \mathrm{g} \mathrm{m}{ }^{-1}\right)$ as required. $P$. aeruginosa was grown in LB or LB-PIA (at 1:1 LB agar and Pseudomonas isolation agar (PIA) (Mathee et al., 1997). The LB-PIA media was used to select for trans conjugants. Tetracycline (Tc, $100 \mu \mathrm{g} \mathrm{ml}^{-1}$ ), and gentamicin $\left(\mathrm{Gm}, 100 \mu \mathrm{g} \mathrm{ml}^{-1}\right.$ ) were used for $P$. aeruginosa. Cultures were grown at $37^{\circ} \mathrm{C}$ unless otherwise stated.

\section{DNA manipulations}

All molecular techniques were performed according to standard protocols (Sambrook et al., 1989). DNA sequencing was performed at Genewiz. Inc. (South Plainfield, NJ). All primers (Table 2.2) used in the experiments were synthesized and supplied by Integrated DNA Technologies (Coralville, IA).

\section{Triparental mating}

Plasmids and cosmids were conjugated into $P$. aeruginosa via triparental mating using mobilizer plasmids pRK2013, and pRK600 (Figurski and Helinski, 1979; Finan et al., 1986). Trans conjugants were then selected for on LB-PIA (1:1) plates containing Tc $\left(100 \mu \mathrm{g} \mathrm{ml}^{-1}\right)$ or $\mathrm{Gm}\left(100 \mu \mathrm{g} \mathrm{ml}^{-1}\right)$. 


\section{Isolation of sap mutants}

Thirty-four nonmucoid sap mutants were isolated in a previous study, and a subset of those was characterized earlier (Sautter et al., 2012). One of the uncharacterized mutants, sap27, is the subject of this study.

\section{Complementation of sap27}

En masse complementation was performed using the minimal tiling path (MTP) library (PAO1 library that contains 336 clones covering $93.7 \%$ of the genome) obtained from Paul Phibbs, East Carolina University (Huang et al., 2000). The 334 clones were previously pooled into four donor pools, each of which originated from the four-microtiter plates (MTP1-MTP96, MTP97-192, MTP193-MTP288, and MTP289-MTP334) used for the library's storage (Sautter et al., 2012). To score for mucoidy, LB:PIA plates with and without glycerol were used to reduce false positives. After one-two days of growth at $37^{\circ} \mathrm{C}$, colonies were restreaked to confirm the mucoid phenotype. The identity of the cosmid was confirmed as described previously (Sautter et al., 2012). The ability of the cosmid pMO012217 to complement was further confirmed by using the original MTP strain MTP36 (Table 2.1).

Table 2.1. Bacterial strains and plasmids used in this study

\begin{tabular}{|c|c|c|}
\hline Strains/plasmids & Genotype/description & References \\
\hline \multicolumn{3}{|l|}{ Strains } \\
\hline \multicolumn{3}{|l|}{ E. coli } \\
\hline DH5 $\alpha$ & fhuA2 $\Delta(\arg F-l a c Z) U 169$ phoA & New \\
\hline & 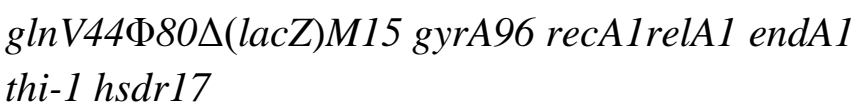 & $\begin{array}{l}\text { England } \\
\text { Biolabs }\end{array}$ \\
\hline MTP36 & $\begin{array}{l}\mathrm{Tc}^{\mathrm{R}} ; \mathrm{pMO} 012217 \text { (PAO1 fragment } 637674- \\
658948)\end{array}$ & $\begin{array}{l}\text { (Huang et } \\
\text { al., 2000) }\end{array}$ \\
\hline
\end{tabular}




\begin{tabular}{|c|c|c|}
\hline \multicolumn{3}{|c|}{ P. aeruginosa } \\
\hline PAO1 & $\mathrm{Alg}^{-} ;$Prototypic strain, nonmucoid & $\begin{array}{l}\text { (Holloway, } \\
1955)\end{array}$ \\
\hline PDO300 & $\mathrm{Alg}^{+} ;$PAOтисA22, constitutively mucoid & $\begin{array}{l}\text { (Mathee et } \\
\text { al., 1999) }\end{array}$ \\
\hline PKM800 & $\begin{array}{l}\mathrm{Alg}^{-} ; \text {mucA22 algO107 (a derivative of PDO300 } \\
(\operatorname{sap} 22))\end{array}$ & $\begin{array}{l}\text { Previously } \\
\text { called } \\
\text { algO96; } \\
\text { (Sautter et } \\
\text { al., 2012) }\end{array}$ \\
\hline PKM817 & $\begin{array}{l}\mathrm{Alg}^{-} ; \text {mucA22 algO107 (a derivative of PDO300 } \\
\text { (sap27)) }\end{array}$ & This study \\
\hline PKM832 & $\begin{array}{l}\mathrm{Alg}^{-} ; \Delta \operatorname{alg} O(\mathrm{PAO} 1 \text { derivative with a clean in- } \\
\text { frame deletion of } \operatorname{alg} O)\end{array}$ & $\begin{array}{l}\text { PAO } \Delta a \lg O \text {; } \\
\text { HK403; } \\
\text { This study }\end{array}$ \\
\hline PKM833 & $\begin{array}{l}\mathrm{Alg}^{-} ; \text {mucA22 } \triangle a l g O(\mathrm{PDO} 300 \text { derivative with a } \\
\text { clean in-frame deletion of } \operatorname{alg} O)\end{array}$ & $\begin{array}{l}\text { PDO } \Delta \text { lagO; } \\
\text { HK404; } \\
\text { This study }\end{array}$ \\
\hline PKM834 & $\begin{array}{l}\mathrm{Alg}^{-} ; \Delta \operatorname{alg} W(\mathrm{PAO} 1 \text { with clean in-frame deletion of } \\
\operatorname{alg} W)\end{array}$ & $\begin{array}{l}\text { PAO } \Delta a l g W \\
\text { HK409; } \\
\text { This study }\end{array}$ \\
\hline PKM835 & $\begin{array}{l}\mathrm{Alg}^{-} ; \text {mucA22 } \Delta a \lg W(\mathrm{PDO} 300 \text { derivative with a } \\
\text { clean in-frame deletion of } \operatorname{alg} W)\end{array}$ & $\begin{array}{l}\text { PDO } \Delta \text { algW; } \\
\text { HK410; } \\
\text { This study }\end{array}$ \\
\hline \multicolumn{3}{|l|}{ Plasmids } \\
\hline $\mathrm{pAlgO}$ & $\mathrm{Tc}^{\mathrm{R}}$; derivative of $\mathrm{pVS} 1$ with algO & $\begin{array}{l}\text { pRTS6000; } \\
\text { (Sautter et } \\
\text { al., 2012) }\end{array}$ \\
\hline pLAFR3 & $\mathrm{Tc}^{\mathrm{R}}$; Cloning vector & $\begin{array}{l}\text { (Staskawicz } \\
\text { et al., 1987) }\end{array}$ \\
\hline pRK600 & $\begin{array}{l}\mathrm{Cm}^{\mathrm{R}} ; \text { colE1 tra }{ }^{+} r k 2 \mathrm{mob}^{+} \text {; Helper plasmid for } \\
\text { conjugation }\end{array}$ & $\begin{array}{l}\text { (Finan } e t \\
\text { al., 1986) }\end{array}$ \\
\hline pRK2013 & $\begin{array}{l}\mathrm{Km}^{\mathrm{R}} ; \text { colE1 } \mathrm{tra}^{+} r k 2 \mathrm{mob}^{+} \text {; Hplasmid for } \\
\text { conjugation }\end{array}$ & $\begin{array}{l}\text { (Figurski } \\
\text { and } \\
\text { Helinski, } \\
\text { 1979) }\end{array}$ \\
\hline pMQ72 & $\begin{array}{l}\mathrm{Gm}^{\mathrm{R}} \text {; colE1; Expression vector containing } \\
\text { arabinose promoter }\end{array}$ & $\begin{array}{l}\text { (Shanks et } \\
\text { al., 2006) }\end{array}$ \\
\hline
\end{tabular}




\begin{tabular}{|c|c|c|}
\hline pEXG2 & $\begin{array}{l}\mathrm{Gm}^{\mathrm{R}} ; \text { colE1, oriT mob } \text { sacB }^{+} ; P . \text { aeruginosa } \\
\text { suicide vector with sucrose counterselection }\end{array}$ & $\begin{array}{l}\text { (Rietsch et } \\
\text { al., 2005) }\end{array}$ \\
\hline pMQ30 & $\begin{array}{l}\mathrm{Gm}^{\mathrm{R}} ; \text { colE } 1 \text {, oriT } \operatorname{sacB}^{+} ; P \text {. aeruginosa suicide with } \\
\text { sucrose counter selection }\end{array}$ & $\begin{array}{l}\text { (Shanks et } \\
\text { al., 2006) }\end{array}$ \\
\hline pSP50 & $\begin{array}{l}\mathrm{Gm}^{\mathrm{R}} \text {; colE1; A pMQ72 derivative with a } \sim 5.01-\mathrm{kb} \\
\text { fragment containing lptD operon downstream of } \\
\mathrm{P}_{\mathrm{BAD}}\end{array}$ & $\begin{array}{l}\text { pLptD- } \\
\text { operon; This } \\
\text { study }\end{array}$ \\
\hline pSP51 & $\begin{array}{l}\mathrm{Gm}^{\mathrm{R}} \text {; colE1; A pMQ72 derivative with a } \sim 2.77-\mathrm{kb} \\
\text { fragment containing } l p t D \text { downstream of } \mathrm{P}_{\mathrm{BAD}}\end{array}$ & $\begin{array}{l}\text { pLptD; This } \\
\text { study }\end{array}$ \\
\hline pSP155 & $\begin{array}{l}\mathrm{Gm}^{\mathrm{R}} \text {; colE1; A pMQ72 derivative with a } \sim 1.29-\mathrm{kb} \\
\text { fragment containing surA downstream of } \mathrm{P}_{\mathrm{BAD}}\end{array}$ & $\begin{array}{l}\text { pSurA; } \\
\text { This study }\end{array}$ \\
\hline pSP156 & $\begin{array}{l}\mathrm{Gm}^{\mathrm{R}} \text {; colE1; A pMQ72 derivative with a } \sim 0.98-\mathrm{kb} \\
\text { fragment containing } p d x A \text { ORF downstream of } \mathrm{P}_{\mathrm{BAD}}\end{array}$ & $\begin{array}{l}\text { pPdxA; } \\
\text { This study }\end{array}$ \\
\hline pSP157 & $\begin{array}{l}\mathrm{Gm}^{\mathrm{R}} \text {; colE } 1 \text {; A pMQ72 derivative with a } \sim 4.04-\mathrm{kb} \\
\text { fragment containing } l p t D \text {-surA downstream of } \mathrm{P}_{\mathrm{BAD}}\end{array}$ & $\begin{array}{l}\text { pLptD- } \\
\text { SurA; This } \\
\text { study }\end{array}$ \\
\hline pSP158 & $\begin{array}{l}\mathrm{Gm}^{\mathrm{R}} \text {; colE1; A pMQ72 derivative with a } \sim 2.37-\mathrm{kb} \\
\text { fragment containing } \text { optP downstream of } \mathrm{P}_{\mathrm{BAD}}\end{array}$ & $\begin{array}{l}\text { pOptP; } \\
\text { This study }\end{array}$ \\
\hline pSP160 & $\begin{array}{l}\mathrm{Gm}^{\mathrm{R}} \text {; colE1; A pMQ72 derivative with a } \sim 0.62-\mathrm{kb} \\
\text { containing lptE downstream of } \mathrm{P}_{\mathrm{BAD}}\end{array}$ & $\begin{array}{l}\text { pLptE; } \\
\text { This study }\end{array}$ \\
\hline pSP161 & $\begin{array}{l}\mathrm{Gm}^{\mathrm{R}} ; \text { colE1; A pSP157 derivative with lptE ORF } \\
\text { from pSP160 subcloned downstream of lptD-surA } \\
\text { creating an operon with lptD-surA-lptE downstream } \\
\text { of } \mathrm{P}_{\mathrm{BAD}}\end{array}$ & $\begin{array}{l}\text { pLptD- } \\
\text { SurA-LptE; } \\
\text { This study }\end{array}$ \\
\hline pSP162 & $\begin{array}{l}\mathrm{Gm}^{\mathrm{R}} \text {; colE1; A pMQ72 derivative with a } 2.77-\mathrm{kb} \\
\text { containing } l p t D \text { fused to six histidine residues at } \mathrm{C} \text { - } \\
\text { terminus and cloned downstream of } \mathrm{P}_{\mathrm{BAD}}\end{array}$ & $\begin{array}{l}\text { pLptD-His6; } \\
\text { This study }\end{array}$ \\
\hline
\end{tabular}

\section{Mutagenesis of pMO012217}

The sap27 mutant strain with the pMO012217 cosmid has the mucoid phenotype. This phenotype in the trans conjugants would be lost if the gene responsible for the complementation in the cosmid is disrupted by transposon mutagenesis. To accomplish this, the pMO012217 cosmid was mutagenized in vitro using the EZ::TN transposon according to the manufacturer's protocol. A 200 nanogram aliquot of pMO012217 was subjected to the EZ::Tn5 <KAN-2> insertion reaction for six hours (Epicenter 
Biotechniques, Madison, WI) followed by transformation into E. coli DH5 $\alpha$ cells. The transformants were cultured on a plate-containing $\mathrm{Km}\left(30 \mu \mathrm{g} \mathrm{ml}^{-1}\right)$. More than 500 transformants were pooled as the donors for triparental mating with sap27. The trans conjugants were screened for the non-mucoid phenotype. Then, the mutagenized cosmid was isolated from $\operatorname{sap} 27$, and the transposon junction was sequenced using the KAN-2 FP1 primer (Table 2.2) to identify the disrupted ORF.

Table 2.2. Primers used in this study

\begin{tabular}{|c|c|}
\hline Primers Name & Sequences $\left(5^{\prime}-3^{\prime}\right)$ \\
\hline SP_OptP_(F) & $\begin{array}{l}\text { ACCCGTTTTTTTGGGCTAGCGAATTCGAGCTCG } \\
\text { GTACCCGGGGAAGGAGATATACATATGAAGCAC } \\
\text { CACTACCGCCT }\end{array}$ \\
\hline SP_OptP_(R) & $\begin{array}{l}\text { AATCTTCTCTCATCCGCCAAAACAGCAAGCTTG } \\
\text { CATGCCTGCAGTCAGAAGTCGTAGCGCAGCGA }\end{array}$ \\
\hline SP_Seq_OptP1 & CTATAATCACGGCAG \\
\hline SP_Seq_OptP2 & ACAACGTCTACCTGG \\
\hline SP_Seq_OptP3 & CGCCAAGGTCGAGCG \\
\hline SP_Seq_OptP4 & CTGATTTAATCTGTA \\
\hline SP_Seq_OptP5 & CCGCAGGGCACGGGG \\
\hline SP_Seq_OptP6 & GACCTTCCTGCGCTG \\
\hline SP_Seq_OptP7 & TGCCCTGCGGACCGC \\
\hline SP_Seq_pMQ72_(F) & CTAACCAAACCGGTAACCCC \\
\hline SP_Seq_pMQ72_(R) & AATTGATGCCTGGCAGTTCC \\
\hline SP_LptD_Operon_(F) & $\begin{array}{l}\text { ACCCGTTTTTTTGGGCTAGCGAATTCGAGCTCG } \\
\text { GTACCCGGGGAAGGAGATATACATATGGCAGTG } \\
\text { AAATCCCTCGTG }\end{array}$ \\
\hline SP_LptD_Operon_(R) & $\begin{array}{l}\text { AATCTTCTCTCATCCGCCAAAACAGCCAAGCTT } \\
\text { GCATGCCTGCAGTCAGCAGCGGCTGGCCGCCATC } \\
\text { TG }\end{array}$ \\
\hline SP_LptD_(F) & $\begin{array}{l}\text { ggctaggaattcctaggagatataccATGGCAGTGAAATCCCTC } \\
\text { GTGTTTCGAA }\end{array}$ \\
\hline SP_LptD_(R) & acgttcatcccgggTTACATAGCTTGATCTTCACGTTGACG \\
\hline SP_LptD-SurA_(F) & $\begin{array}{l}\text { ggctaggaattcctaggagatataccATGGCAGTGAAATCCCTC } \\
\text { GTGTTTCGAA }\end{array}$ \\
\hline
\end{tabular}




\begin{tabular}{ll}
\hline SP_LptD-SurA_(R) & acgttcatcccgggTCACTGCTTGATTTCCACGTAGGCCTC \\
SP_SurA_(F) & ACCCGTTTTTTTGGGCTAGCGAATTCGAGCTCG \\
& GTACCCGGGGAAGGAGATATACATATGAAGATC \\
& AAGCTATGTAAC \\
SP_SurA_(R) & AATCTTCTCTCATCCGCCAAAACAGCCAAGCTT \\
& GCATGCCTGCAGTCACTGCTTGATTTCCACGTAG \\
& GCCTC \\
SP_PdxA_(F) & ACCCGTTTTTTTGGGCTAGCGAATTCGAGCTCG \\
& GTACCCGGGGAAGGAGATATACATATGAGCCTG \\
& CGCTTCGCGCTC \\
SP_PdxA_(R) & AATCTTCTCTCATCCGCCAAAACAGCCAAGCTT \\
& GCATGCCTGCAGTCAGCAGCGGCTGGCCGCCATC \\
& TG \\
SP_JuntSurAPdxA_(R) & TTGATCACGCCCTTGTGCA \\
SP_LptD_Seq1 & AAATCCCGCAGTGCGGACTA \\
SP_LptD_Seq2 & ACAACGCCGAGTACGTGATCCA \\
SP_LptD_Seq3 & AAGGCGAGTTCCGCTACCTGA \\
SP_LptD_Seq4 & GGAATGCAATTCACCTATGGCA \\
SP_LptD_Seq5 & CCATGTACTTGTACGTTCCAT \\
SP_LptD_Seq6 & GGTAGCGCCATCTTCCACTA \\
SP_LptD_Seq7 & GTAGGCAACAAGACGGAAATG \\
SP_LptD_Seq8 & CGGTGGCAGGTTGGCATT \\
SP_LptD_Seq9 & TAGTCCGCACTGGGGGATTT \\
SP_LptD_seq10 & GGGAGCAATCCCAG \\
SP_SurA_Seq1 & GTGCACCAGACGCTG \\
SP_SurA_Seq2 & GGCCAATATCCTGATC \\
SP_SurA_Seq3 & CTGCTCAAGCCCAGC \\
SP_SurA_Seq4 & CCGTCGCGGGTGAGGG \\
SP_SurA_Seq5 & GCCGAACTGCGAGCGG \\
SP_PdxA_Seq1 & TTCCGGCCATACCGAA \\
SP_PdxA_Seq2 & CAGACCAGGATGCGCGGAT \\
Cos-1 & CGCCCTCTGGTAAGGTTG \\
pLA2 & CCTGTCTCTTGATCAAGATC \\
KAN-2FP-1 & ACCTACAACAAAGCTCTCATGAACC \\
HK_AlgW_DR & CCAGGCAAATTCTGTTTTATCAGACCGCTTCTGCGT \\
& TCTGATTGGATGAAAGGTTCCGCA \\
HK_AlgW_DF & CCCCAAGGATTTTCCCGATGCTAGCTAACTAGTCC \\
& CCGTCATTGAAAACGGC \\
\hline &
\end{tabular}




\begin{tabular}{ll}
\hline HK_AlgW_UR & GCCGTTTTCAATGACGGGGACTAGTTAGCTAGCAT \\
& CGGGAAATCCTTGGGG \\
HK_AlgW_UF & GGAATTGTGAGCGGATAACAATTTCACACAGGAA \\
& ACAGCTGTCTGCTCGGAAACCTCGCC \\
HK_AlgW_P1_FW_Se & TAATGGACGGCTTCCCCCTA \\
q & \\
HK_AlgW_P2_RE_Seq & CAGGGAGTCATCGTCCGCCA \\
HK_AlgW_P3_FW_Ex & AGCGCCTGTACCCCGTAGC \\
t & \\
HK_AlgW_P3_RE_Ext & GCTGTTTCCGGACGTGACCTAC \\
SP_AlgO_(F) & ATTAATAAGCTTGCTGCCTCCATAGTGGG \\
SP_AlgO_(R) & AATTATAAGCTTTTATGACGCTCCCGCTGAACT \\
CC_AlgO_P1_(F) & AAGCTTAGCAACTCCAGGTTCAACCCGG \\
CC_AlgO_P1_(R) & GCTAGCGCTGATTCGACTGATCGCGGAGT \\
CC_AlgO_P2_(F) & GCTAGCGTTCAGCGGGAGCGTCATAAA \\
CC_AlgO_P2_(R) & GGATCCCGAGGTGCGAACGTCGACCGCG \\
CC_AlgO_Seq1 & GGCGCTGCCGACGGAGAC \\
CC_AlgO_Seq2 & GCAACAGGTTCGACGACT \\
CC_AlgO_Seq3 & CGGACAACGCGGAAAACT \\
CC_AlgO_Seq4 & CTACAAGCTCGGCGTGAT \\
CC_AlgO_Seq5 & ACCGTGCAGACCATCCAGC \\
CC_AlgO_Seq6 & CCAGTTGGCCATGGAGAACG \\
CC_AlgO_Seq7 & CATCGACCAGGGCCCGACCG \\
CC_AlgO_Seq8 & GTGTCCCAGGGCATCGAGTCG \\
CC_AlgO_Seq9 & TGGCGATGACCTGCTCGCGG \\
CC_AlgO_Seq10 & GTCCAGGTAGAAGGCGGGCA \\
CC_AlgO_Seq11 & GAGCCGACCTTTCAGGCGG \\
SP_LptE_(F) & ggctagcccgggtaggagatataccATGAAACGTATCCTGACCA \\
SP_LptE_(R) & GCGCCGCGC \\
SP_LptD-HindII_6xHis & cagccaagcttTCACGGGGTGGGGAACTCGATCGG \\
(R) & TTGATCTTCACGTTGACG \\
\hline &
\end{tabular}




\section{Alginate assay}

In brief, cells from a single plate were harvested after growth in LB for $36 \mathrm{hr}$ and resuspended in $5 \mathrm{ml}$ PBS (pH 7.4) and the OD600 was determined. Then, the cell suspension was centrifuged at $12,000 \mathrm{X} g$ for 30 minutes. The supernatant was collected, and the alginate was precipitated using $5 \mathrm{ml}$ of $2 \%(\mathrm{w} / \mathrm{v})$ cetylpyridinium chloride. The mixture was centrifuged at $12,000 \mathrm{X} g$ for 10 minutes and the pellet was resuspended in 5 $\mathrm{ml}$ of $1 \mathrm{M} \mathrm{NaCl}$ and $5 \mathrm{ml}$ of cold $\left(-20{ }^{\circ} \mathrm{C}\right)$ isopropanol. The suspension was again centrifuged at $12,000 \mathrm{Xg}$ for 10 minutes and the pellet was dried at $37{ }^{\circ} \mathrm{C}$ for 30 minutes to evaporate any residual isopropanol. Finally, the pellet was resuspended in $5 \mathrm{ml}$ of $0.9 \%$ (w/v) $\mathrm{NaCl}$. To determine the alginate concentration, a set of standards was made with sodium alginate (Sigma-Aldrich, St. Louis, MO). The amount of alginate was determined using a borate/carbazole method. A 70- $\mu$ l-alginate sample was mixed with $600 \mu \mathrm{l}$ of icecold borate solution then immediately placed on ice and was followed by the addition of $20 \mu \mathrm{l}$ of $0.1 \%$ carbazole and mixed briefly. Then the mixture was heated for 30 minutes at $55{ }^{\circ} \mathrm{C}$, and the absorbance was measured at $530 \mathrm{~nm}$. The absorbance was compared with standards to determine the alginate concentration which was expressed in $\mathrm{mg}$ per $\mathrm{ml}$ supernatant and normalized to the bacterial OD $600=0.41$ (Qiu et al., 2007). All the alginate assays were performed in triplicate.

\section{Construction of plasmids containing the ORF lptD-operon, surA, $p d x A$, and optP}

The ORFs surA, $p d x A$ and $o p t P$ were amplified from the genomic DNA of PAO1 using standard procedures (Sambrook et al., 1989). The primer pairs SP_LptD_Operon_(F), and SP_LptD_Operon_(R), SP_SurA_(F) and SP_SurA_(R), 
SP_PdxA_(F)-SP_PdxA_(R), and SP_OptP_(F)-SP_OptP_(R), (Table 2.2) were used to amplify the lptD-operon $(5.01 \mathrm{~kb})$, surA $(1.29 \mathrm{~kb}), \operatorname{pdxA}(0.98 \mathrm{~kb})$, and optP $(2.373 \mathrm{~kb})$, respectively. The forward primers used contained a ribosome-binding sequence upstream of the start codon of the respective ORFs as well as 45-bp tails (shown in bold, Table 2.2) that target recombination downstream of the $\mathrm{P}_{\mathrm{BAD}}$ promoter on pMQ72 that has pRO1600 and colEl origins of replication (Shanks et al., 2006). The respective reverse primers also have 44-bp tails (shown in bold, Table 2.2) that target recombination upstream of T1T2 transcriptional terminators present in the vector (Shanks et al., 2006). The vector pMQ72 was digested with restriction enzyme SmaI. Then the digested vector pMQ72 along with PCR product were co-transformed into overnight cultures of Saccharomyces cerevisiae (Shanks et al., 2006). The transformed cells were individually tested for the desired construct by colony PCR using the primer pair SP_Seq_pMQ72_(F)SP_Seq_pMQ72_(R) for the presence of an insert (Shanks et al., 2006; Shanks et al., 2009). E. coli DH5 $\alpha$ was then transformed with the recombinant pMQ72 plasmids containing their respective ORFs and screened for Gm resistance. The amplified ORFs from the recombinant plasmids were sequenced to ensure fidelity (Table 2.2). The plasmids with $\mathrm{P}_{\mathrm{BAD}}$ driving the expression of $l p t D$-operon, surA, $p d x A$, and optP are pSP50, pSP155, pSP156, and pSP158, respectively (Table 2.1). These plasmids henceforth will be referred to as pLptD-operon, $\mathrm{pSurA}, \mathrm{pPdx} \mathrm{A}$, and $\mathrm{pOptP}$, respectively.

\section{Construction of plasmids containing the $\operatorname{lptD}, \operatorname{surA}$ and $\operatorname{lptE}$}

The ORFs, lptD (2.77 kb), lptD-surA (4.048 kb), and lptE (0.62 kb), were amplified from PAO1 genomic DNA using the primer pairs SP_LptD_(F)—SP_LptD_(R), 
SP_LptD-SurA_(F)-SP_LptD-SurA_(R), and $\quad$ SP_LptE_(F)-SP_LptE_(R), respectively (Table 2.2). The amplified product $l p t D$ and $l p t D$-surA were digested with the enzymes EcoRI and XmaI then ligated downstream of PBAD in pMQ72 (Shanks et al., 2006) cut with same restriction enzymes. Then, the resulting plasmids harboring $l p t D$ and $l p t D$ surA genes were named as pSP51 and pSP157, respectively.

The PCR-amplified product of $l p t E$ was restriction digested by enzymes XmaI and Hindlll then ligated downstream of $\mathrm{P}_{\mathrm{BAD}}$ in $\mathrm{PMQ} 72$ digested with same restriction enzymes. Then the $l p t E$ harboring plasmid was named as pSP160. The lptE fragment of pSP160 was subcloned into plasmid pSP157 downstream of lptD-surA using restriction enzymes XmaI and HindIII to create pSP161. The plasmid pSP161 contains lptD-surA-lptE under the control of $\mathrm{PBAD}_{\mathrm{BAD}}$

All the constructs were sequenced to ensure fidelity using the appropriate primers shown in Table 2.2. The constructs, pSP51, pSP157, pSP160 and pSP161, henceforth will be referred to as pLptD, pLptD-SurA, pLptE and pLptD-SurA-LptE, respectively.

\section{His-tagging of LptD}

A 2.77-kb fragment with $l p t D$ was amplified from PAO1 genomic DNA using the primer pairs SP_LptD (F) and SP_LptD-6xHis (R) (Table 2.2). The latter primer has six histidine codons added in frame to the 3 ' end of the coding sequence of $l p t D$. The amplified product with lptD-His6 was then ligated downstream of $\mathrm{P}_{\mathrm{BAD}}$ in $\mathrm{pMQ} 72$ to construct pSP162 (pLptD-His6). The cloned fragment was sequenced for fidelity. The plasmid pLptD-His6 was introduced into the sap27 strain by standard electroporation (Choi et al., 2006). 


\section{Western blotting}

Cells were grown to an $\mathrm{OD}_{600}$ of 0.4 and induced with $2 \%$ arabinose for 2 hours at $25{ }^{\circ} \mathrm{C}$. Then the cells were harvested by centrifugation at the speed of $5,000 \mathrm{rpm}$ at $4{ }^{\circ} \mathrm{C}$ for 10 minutes. The pellets were resuspended in $10 \mathrm{ml}$ lysis buffer $(20 \mathrm{mM}$ Tris [pH 7.5], 0.1 $\mathrm{M} \mathrm{NaCl}, 1 \mathrm{mM}$ EDTA, $1 \mathrm{X}$ Roche complete protease inhibitor cocktail, lysozyme [0.5 $\mathrm{mg} / \mathrm{ml}]$ ). After lysis by sonication (15 cycles of 10 -s pulse on and 30-s pulse off; amplitude, $40 \%$ ), the cell lysates were centrifuged $5,000 \mathrm{rpm}$ for 10 minutes at $4{ }^{\circ} \mathrm{C}$. Two $\mathrm{ml}$ of the cell lysate from each sample was saved as the total cell fraction, and remaining were further fractioned by ultracentrifugation at $36,000 \mathrm{rpm}$ (SW $41 \mathrm{Ti}$ rotor) for one hour at $4^{\circ} \mathrm{C}$. The supernatants were saved as soluble (cytoplasmic and periplasmic) fraction whereas pellets were resuspended with $1 \mathrm{ml}$ of membrane buffer $(20 \mathrm{mM}$ Tris- $\mathrm{Cl}$ [pH7.5], $0.2 \%$ Sodium lauroyl sarcosinate [Sarkosyal; Fluka], $0.1 \%$ Sodium dodecyl sulfate [SDS]). One hundred $\mathrm{ml}$ of the whole cell, periplasmic/cytoplasmic and membrane fractions were aliquoted and stored at $-80^{\circ} \mathrm{C}$. The concentration of protein was determined by the bicinchoninic acid method (Smith et al., 1985). Ten micrograms of total protein were separated on a $12 \%$ SDS-polyacrylamide gel. The presence of LptD and $\sigma^{70}$ (loading control) was detected using anti-His 6 (Thermo Scientific, Rockford, IL) and anti- $\sigma^{70}$ (Neoclone Biotechnologies International, Madison, WI) antibodies. The fluorescent secondary antibody (Anti-mouse IgG $(\mathrm{H}+\mathrm{L})$ (DyLight ${ }^{\mathrm{TM}} 680$ Conjugate) was purchased from Cell Signaling Technology, Inc (Danvers, MA). The fluorescence was detected using Odyssey CLx near Infrared Imaging System (LI-COR Biosciences, Lincoln, NE). 


\section{Construction of $\triangle a l g O$ and $\triangle a l g W$ strains}

An in-frame deletion of algO was constructed using overlap extension PCR and homologous recombination as previously described (Horton et al., 1990). The upstream and downstream flanking regions of algO using the primer pairs CC_AlgO_P1_(F)— CC_AlgO_P1_(R) and CC_AlgO_P2_(F)—CC_AlgO_P2_(R), respectively were amplified and fused to construct the deletion (Table 2.2). The PCR products were then ligated through another round of PCR and cloned into the suicide vector pEXG2 (Rietsch et al., 2005). The recombinant plasmid was introduced into PAO1 and PDO300, where the deletion replaced the wild-type $\operatorname{alg} O$ on the chromosome via homologous recombination using a two-step process, involving screening by Gm sensitivity followed by sucrose resistance. PCR amplification of the region spanning the deletion and sequencing confirmed the absence of algO. These deletion mutants were called PAO $\triangle a l g O$ and PDO $\triangle a \lg O$.

A gene-splicing method using the yeast system was used to generate a precise inframe deletion of algW in P. aeruginosa (Shanks et al., 2006). Upstream (P1) and downstream (P2) flanking regions of $\operatorname{alg} W$ were amplified from PAO1 genomic DNA by PCR using the primer pairs HK_AlgW_UF-HK_AlgW_UR and HK_AlgW_DFHK_AlgW_DR (Table 2). The primers HK_AlgW_UF and HK_AlgW_DR had regions of homology to target recombination of $\mathrm{P} 1$ and $\mathrm{P} 2$ with pMQ30 vector (Shanks et al., 2006). The primer pairs also had complementary sequences at the $3^{\prime}$ end to facilitate joining to create the P3 fragment, as well as stop codons (5'-CTAGTTAGCTAG-3') to prevent translational read through. The pMQ30 vector has double selection markers URA3 for yeast and $\mathrm{Gm}^{\mathrm{R}}$ for E. coli (Shanks et al., 2006). Yeast cells were transformed with the P1, 
P2 and BamHl-digested linearized pMQ30 using standard protocols and colonies were selected on sucrose-uracil plates. The yeast colonies were checked for the presence of the P3 construct for algW deletions by amplifications using primer pairs HK_AlgW_UFHK_AlgW_DR. Yeast DNA was isolated from the positive colonies as previously described (Shanks et al., 2009). E. coli DH5 $\alpha$ was transformed with the recombinant pMQ30 plasmids containing P3 and screened for Gm resistance. The amplified P3 from the recombinant plasmids were sequenced to ensure fidelity. The constructs were then conjugated into PAO1 using triparental mating and screened for single and double crossovers using counter selection with sucrose and $\mathrm{Gm}$, as previously described (Shanks et al., 2006; Shanks et al., 2009). The presence of the gene deletion in all the mutants was confirmed using standard molecular methods. The strain is henceforth referred to as PAO $\Delta a l g W$. Using similar strategies $\Delta a \lg W$ in mucoid PDO300 was constructed and named PDO $\Delta a \lg W$.

\section{RNA isolation and cDNA synthesis}

RNA was isolated from $P$. aeruginosa wild-type PAO1 as described previously (Balasubramanian et al., 2014). Briefly, 10-ml overnight cultures grown in LB broth at $37^{\circ} \mathrm{C}$ were washed with $0.85 \%$ saline solution to remove spent media. Then the cells were sub-cultured in $15 \mathrm{ml}$ by inoculating the culture in $\mathrm{LB}$ media at an $\mathrm{OD}_{600}$ of 0.01 at $37^{\circ} \mathrm{C}$ at $200 \mathrm{rpm}$. Subsequently, the cells were harvested when the culture reached an $\mathrm{OD}_{600}$ of 0.5-0.6. The RNA was stabilized by addition of phenol-ethanol mixture as described previously (Brencic et al., 2009). Stabilized RNA was then isolated using RNeasy Mini Kit (Qiagen Inc, Germantown, MD, USA) as per the manufacturer's protocol. Residual 
genomic DNA contamination was removed using RQ1 RNase-free DNase (Promega, Madison, WI, USA) and RNA was repurified using RNeasy Mini Kit (Qiagen Inc, Germantown, MD, USA) The quality of purified RNA was assessed on a denaturing agarose gel (NorthernMax Gly, Ambion, Life Technologies, Carlsbad, CA) and quantified at $\mathrm{OD}_{260}\left(\right.$ BioTEK$^{\circledR}$, Synergy ${ }^{\mathrm{TM}} \mathrm{HT}$, Winooski, VT, USA). cDNA was then synthesized by annealing NS5 random primers to total purified RNA, and subsequent extension was carried out using SuperScript III reverse transcriptase (Invitrogen ${ }^{\mathrm{TM}}$, Life Technologies, Carlsbad, CA, USA).

\section{Results}

\section{Identification of the sap27 complementing cosmid}

The sap27 mutant is a nonmucoid revertant isolated by growing mucoid PDO300 without aeration (Sautter et al., 2012). The mucoid phenotype in sap 27 was restored via en masse complementation with the PAO1 minimum tiling path (MTP) genomic cosmid library (Huang et al., 2000). The complementing cosmid was extracted and the junction where the $P$. aeruginosa genome fragment is integrated was sequenced to identify the $P$. aeruginosa genes contained in pMO012217 (PAO1 coordinates: 637674 to 658948; Figure 2.1) (Huang et al., 2000). To ensure that was pMO012217 was the complementing cosmid, sap27 was re-transformed by conjugation with E. coli containing the original cosmid clone pMO012217 from the MTP library (MTP36) (Figure 2.3(a)). As negative controls, PAO1 and sap27 were transformed with vector pLAFR3. As expected, PAO1 with the vector remained nonmucoid. The positive controls, the $\mathrm{Alg}^{+}$parent PDO300 with the cosmid and plasmid vectors, remained mucoid when transformed individually. The 
cosmid pMO012217 restored the mucoid phenotype in sap27 (Figure 2.3(a)). The pMO012217 cosmid contained 18 ORFs (Figure 2.1) and did not harbor any known alginate genes indicating that a novel ORF was involved in the restoration of alginate production.

\section{Mapping the gene in the complementing cosmid pMO012217 using transposon (Tn)}

\section{mutagenesis}



Figure 2.1. Genetic map of cosmid pMO012217 and $l$ thD-operon. The cosmid was identified in the en masse complementation of sap27 with the MTP library, which restored the mucoid phenotype. The cosmid contains 18 ORF. The figure of the cosmid was retrieved from pseudomonas.com and reversed to make it easier for observation because the lptD operon lies in negative strand of the chromosome of $P$. aeruginosa. Through sequencing, we identified the transposon was inserted at the end of $l p t D$, which is marked by an orange arrow. The in silico sequence analysis shows that the 3' of the lptD overlaps with 5' end of $s u r A$, which is shown by dotted line [start codon [GTG] of surA begins before stop codon [TAA] of lptD] and stop codon of surA [TGA] and start codon [GTG] of $p d x A$ overlaps [GTGA] also shown by dotted line that originate from intersection of surA and $p d x A$. The operon was cloned into an expression vector pMQ72 and named as pLptD-operon. Plasmids pLptD-operon, $\mathrm{pLptD}$, pSurA, and pPdxA are pMQ72-derived plasmids with lptD-operon, lptD, surA, and $p d x A$ insertions, respectively. The plasmids were constructed as described in the materials and methods. The red arrow shows the transposon insertion site in lptD gene and the genomic position. 
The rationale for the Tn mutagenesis is that the loss of mucoidy in the trans conjugants would be due to the disruption of the complementing ORF present in the cosmid.

To identify the particular ORF responsible for the mucoid conversion, pMO012217 was mutagenized in vitro using the EZ::TN transposon (Epicenter Biotechniques, Madison, WI). The transposon mutant library was introduced into sap27 and screened for the loss of the mucoid phenotype in the trans conjugants. Sequencing of the Tn junction in the constructs that failed to restore the mucoid phenotype using the cos-1 primer (Table 2.2) revealed the insertion to be at the $3^{\prime}$ end of $l p t D$ (PA0595) at position 653,902 of P. aeruginosa PAO1 genome (Figure 2.1). This insertion disrupts the alanine codon at the position 876 of LptD, truncating the protein.

\section{Analysis of the lptD locus}

Analysis of the locus suggests that lptD might be a part of the three-gene operon (Winsor et al., 2016). Thus, the Tn insertion could have a polar effect on the downstream genes, namely surA and $p d x A$. In $E$. coli, it has been shown that SurA is an essential protein for survival in starvation and a major chaperone protein for all outer membrane protein. In E. coli, $\mathrm{PdxA}$ is a NAD-dependent dehydrogenase and is involved in the vitamin $\mathrm{B}_{6}$ biosynthetic pathway (Banks and Cane, 2004; Laber et al., 1999). To verify if lptD-surA $p d x A$ forms an operon in P. aeruginosa, total RNA was isolated from PAO1 in late logphase, and non-specific primers were used to synthesize cDNA for RT-PCR. The presence

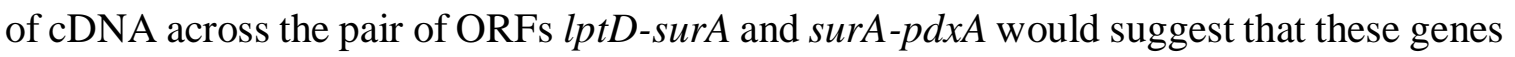
form an operon. The expected PCR products of sizes 1000 bp (Lanes 2, 3 and 5) and 900 bp (Lanes 8, 9 and 11) for lptD-surA and surA-pdxA, respectively, were observed (Figure 
2.2). As expected, there was no product in negative controls when amplification was done without the DNA polymerase (Lanes 7 and 13); or using RNA prepared after DNAse I treatment (Lanes 4 and 10) or without reverse transcriptase (Lanes 6 and 12).

The presence of expected PCR products in the cDNA (Lanes 5 and 11) confirms the existence of mRNA spanning the junctions. These data suggest $\operatorname{lptD}$, surA and $p d x A$ form an operon. Since the Tn is inserted at the $3^{\prime}$ end of the first gene, lptD, at 653,902 in the three-gene operon, it is possible that all three genes may be involved in alginate production (Figure 2.2). Thus, the three ORFs in the lptD-operon alone or combination might be responsible for restoring the mucoid phenotype in sap27. To determine the ORF

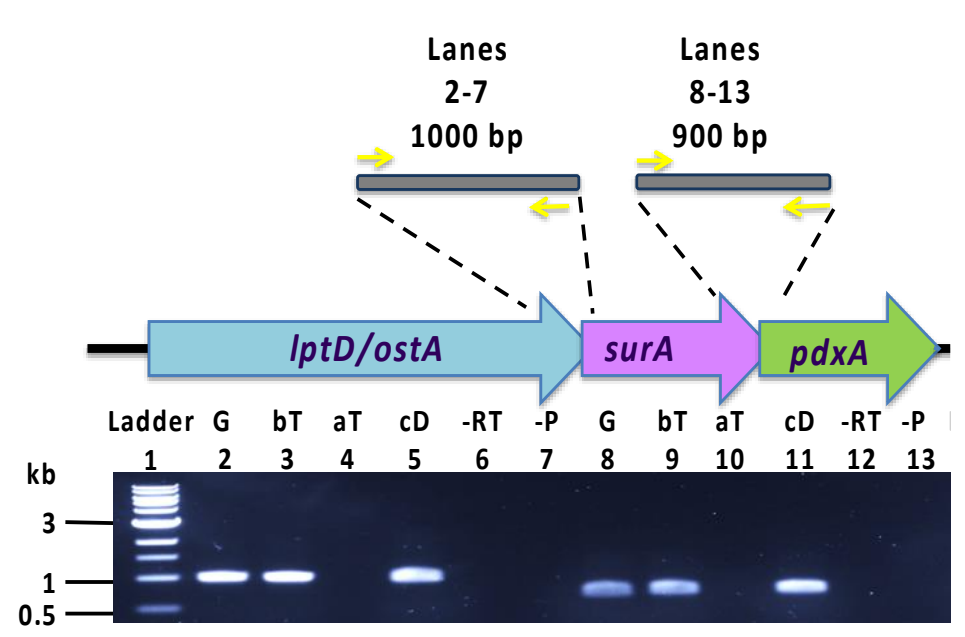

Figure 2.2. Verification of $l p t D$ is part of a three-gene operon. The PCR amplification of the junctions between the genes, lptD-surA and, surA-pdxA with specific primer pairs shown in Table 2. The amplified product was run in $1 \%$ agarose gel. Lane 1, $1 \mathrm{~Kb}$ Ladder $[\mathrm{M}]$ as a marker; Lanes 2-7 and Lanes 8-13 is the PCR products of the junction between lptD-surA and surA-pdxA, respectively. Lanes $2 \&$ 8, PCR products from genomic DNA [G] used as positive control; Lanes 3,4,9, and 10, PCR products before treatment and after treatments (aT) of DNA polymerase; Lanes 5 $\& 11$, PCR products from cDNA [cD]; Lanes $6 \& 12$, PCR amplification without the reverse transcriptase [-RT] used as negative control; Lanes $7 \& 13$, PCR amplification in the absence of DNA polymerase [-P] used as negative control. 
responsible, $l p t D$, surA, and $p d x A$ were individually cloned downstream of the $\mathrm{PBAD}_{\mathrm{B}}$ promoter and the plasmids were named pLptD, pSurA, and pPdxA, respectively (Table 2.1). In addition, the entire operon was also subcloned into the same vector (pMQ72). All

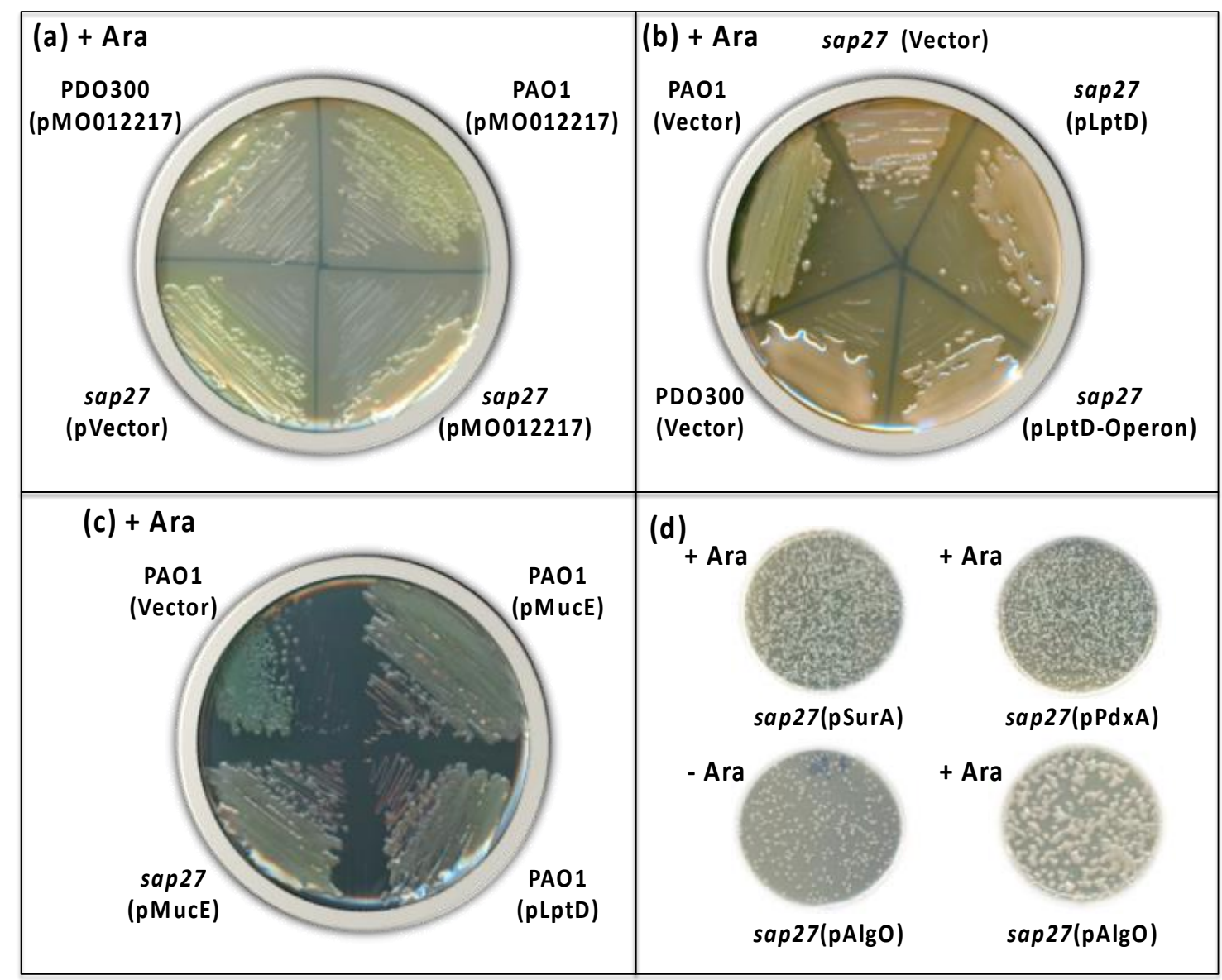

Figure 2.3. Identification and mapping of the gene responsible for restoring mucoid phenotype. Identification of genes responsible for restoring the mucoid phenotype. (a) The cosmid pMO012217 introduced into sap27 restoring the mucoid phenotype. PAO1 and PDO300 were used as negative and positive controls, respectively. The plasmid pLAFR3 was used as a control vector. (b) The plasmids

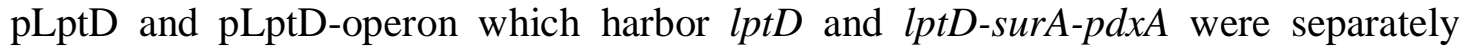
introduced into sap27 restoring the mucoid phenotype. (c) The plasmids, pMucE and pLptD were introduced into PAO1 and sap27 inducing a mucoid phenotype. (d) The plasmids, pSurA, pPdxA and pAlgO were introduced into sap27. Mucoid phenotype was restored only when $\operatorname{sap} 27(\mathrm{pAlgO})$ was induced with arabinose. The strains were plated on LB containing $2 \%$ arabinose (Ara) where indicated and incubated at $37^{\circ} \mathrm{C}$ for 36 hours. 
the constructs were introduced into sap27 and PDO300. The vector (pMQ72) was also introduced into sap27 and PDO300 as negative controls. The plasmid harboring mucE (pMucE) that is known to induce alginate production was introduced into sap27 and PAO1 as positive controls (Figure 2.3(c))(Qiu et al., 2007). After $36 \mathrm{hr}$, the mucoid phenotype in sap27 was restored by the pLptD-operon and $\mathrm{pLptD}$ in the presence of arabinose (2\%) (Figure 2.3(b)) but not by pSurA and pPdxA (Fig. 2.3(d)). As expected, the amount of alginate produced by sap27 in the presence of $l p t D$ and $l p t D$-operon was comparable to PDO300 (Figure. 2.4). These findings suggest that lptD alone is sufficient to restore the mucoid phenotype in sap27.

\section{Co-expression of lptD, surA, and lptE in sap27}

The $20 \mathrm{kDa}$ proper folding of LptD in the outer membrane (Chimalakonda et al., 2011; Chng et al., 2010; Grabowicz et al., 2013). In addition, LptD folding also requires the chaperone protein SurA (Vertommen et al., 2009).One could argue that alginate production in sap 27 is due to stress induced by the accumulation of misfolded LptD as a result of increased expression of $l p t D$ alone without surA and/or $l p t E$. To rule out the potential for LptD misfolding, plasmids with lptD-surA (pLptD-SurA), and lptD-surA-lptE (pLptD-SurA-LptE) under the control of $\mathrm{P}_{\mathrm{BAD}}$ were constructed (Table 2.1). These constructs were introduced individually into sap 27 and the genes were induced with arabinose. Alginate production was restored in sap 27 in the presence of both of the plasmids individually (Figure 2.4). This suggests that the mucoid conversion of sap27 upon lptD overexpression is not due to misfolding of $\mathrm{LptD}$, instead, it is the function of increasing the copy number of $l p t D$. 


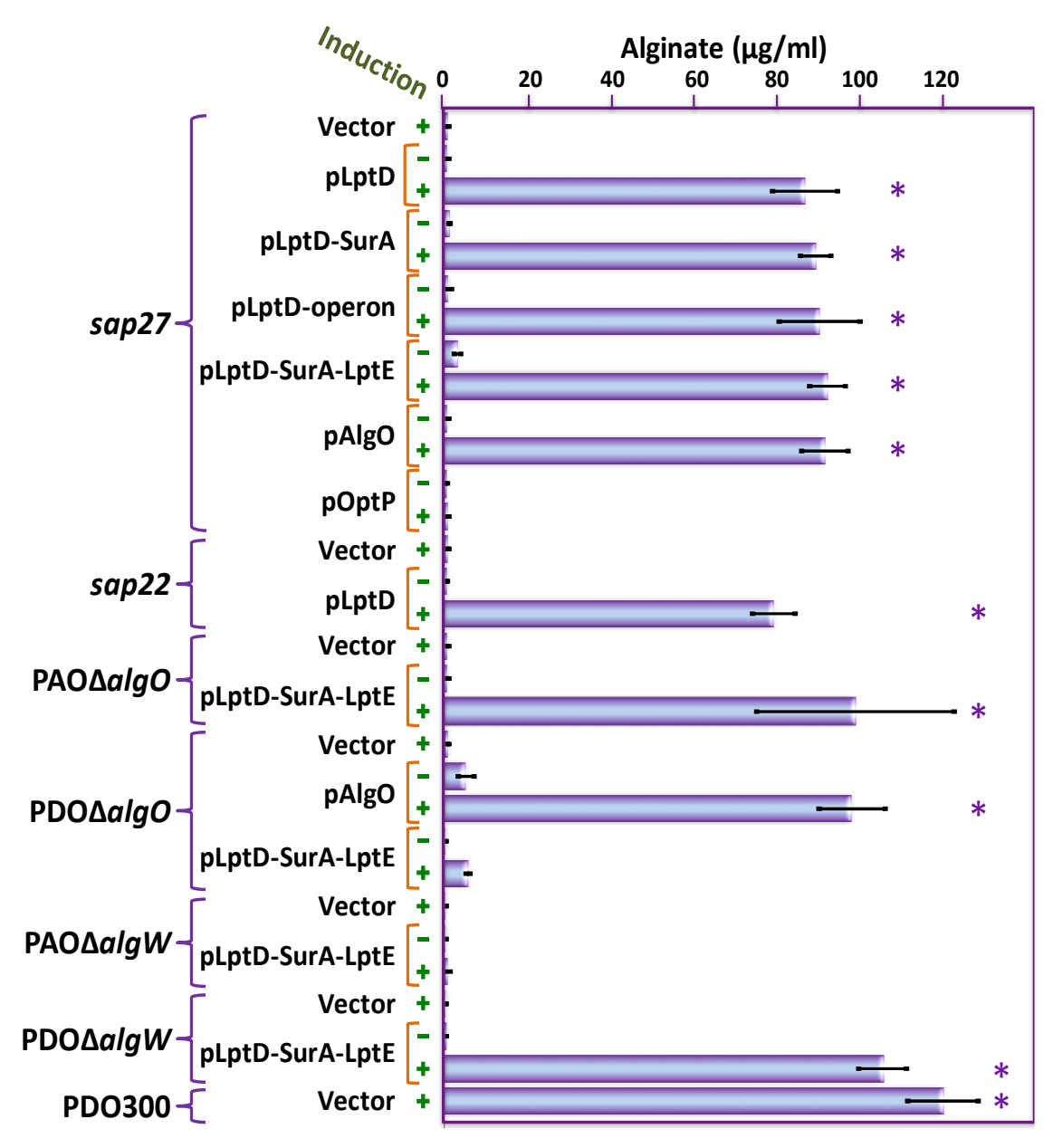

Figure 2.4. Alginate levels in the presence of increased expression of $\mathbf{l p t D}$. Plasmids containing the lptD, lptD-surA, lptD-surA-lptE, optP, oprP, and $\operatorname{alg} O$ were introduced separately into sap27, and strains with clean in-frame deletion of algO [PAO $\triangle$ alg $O$ and $\mathrm{PDO} \Delta \operatorname{alg} O]$ and $\operatorname{alg} W[\mathrm{PAO} \Delta \operatorname{alg} W$ and PDO $\Delta \operatorname{alg} W]$. Cells were grown for 36 hours on LB plates supplemented with gentamycin [100 $\mu \mathrm{g} / \mathrm{ml}$ for selection and $2 \%$ arabinose as an inducer. The alginate level was quantified and normalized to an $\mathrm{OD}_{600}=0.41$ as described in materials and methods. The strains sap27 and PDO300 with vectors were used as the negative and the positive control, respectively. The level of significance $[p-$ value] is less than 0.05 for each set of experiment. The experiments were done in triplicates. 
The latter would then imply that the concentration of LptD will be higher in sap27 if the expression of $l p t D$ is induced. We confirmed this by His-tagging LptD and Westernblotting using anti-His6 antibody (Figure 2.5). In addition, we also demonstrate that the LptD is only found in the insoluble fraction suggesting membrane localization (Figure 2.5).

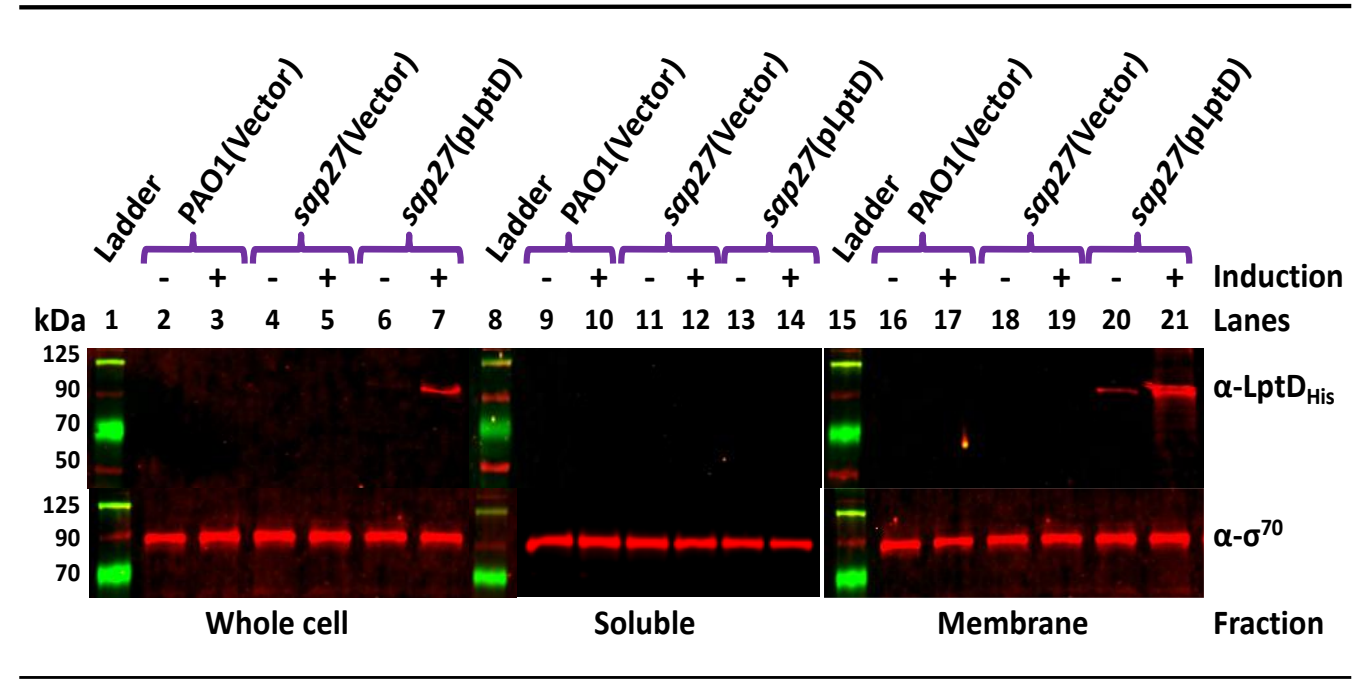

Figure 2.5. Cellular localization of LptD. Equal amounts of total proteins were loaded per well. His-tagged LptD was introduced into sap27 as described in materials and methods. Whole cell fractions were extracted from cells that were uninduced (-) or induced (+) with $2 \%$ arabinose for 3 hours at $25^{\circ} \mathrm{C}$. The induced whole cell extract was further separated into soluble and membrane fraction. The presence of $\mathrm{LptD}$ was shown using anti-His6 antibody. Sigma 70 was used as the loading controls. The whole cell and membrane fractions were probed with unconjugated purified anti-E. coli RNA sigma 70 antibodies. The soluble fraction was probed with conjugated Direct-Blot ${ }^{\mathrm{TM}}$ HRP anti-E. coli RNA sigma 70 antibody, thus the ladder that was run parallel was superimposed in this image.

\section{Expression of optP encoding a large OMP in sap27}

To determine if the mucoid restoration of sap27 is exclusive to lptD, optP (PAO192) was expressed under the $\mathrm{P}_{\mathrm{BAD}}$ promoter in sap27. The protein OptP was chosen 
as it is predicted to be a large outer membrane protein (790 aa) comparable in size to the 924-aa LptD (Winsor et al., 2016). Overexpression of optP did not turn the sap27 strain into the mucoid phenotype and consequently, no alginate was detected (Figure 2.4), suggesting that it is not a general function of large OMPs. This suggests that LptD might have potentially a specific role for alginate production in sap27.

\section{Mapping of the mutation in sap27}

The above finding suggested that sap27 harbors a mutation in $l p t D$. To rule out any promoter mutation that may influence lptD expression, the entire operon including the promoter region was sequenced in sap27 and no mutation was found. This suggested that

\begin{tabular}{|c|c|c|c|c|c|c|c|c|c|}
\hline Amino acid & 106 & 107 & 108 & & & 318 & 319 & 320 & 321 \\
\hline \multirow[t]{2}{*}{ PAO1 } & GCC & GAG & TTC & . & . & CTG & GAT & GAA & . \\
\hline & Ala & Glu & Phe & - & - & Leu & Asp & Glu & • \\
\hline \multirow[t]{2}{*}{ sap27 } & GCC & GAI & GTT & - & • & $\mathrm{CCT}$ & GGA & TGA & \\
\hline & Ala & Asp & Val & . & • & Pro & Gly & * & \\
\hline
\end{tabular}

Figure 2.6. Mapping the algo mutation in sap27. The codon numbers of the wildtype $\operatorname{alg} O$ are shown on top. Sequencing of algO from sap27 revealed an insertion of a nucleotide thymine ( $\mathrm{T}$ ) (shown in red) causing a frame-shift at codon 107 leading to premature translational termination of $\mathrm{AlgO}$ at codon 320 resulting in a truncated protein. The amino acid sequences from codon 107 to 319 share no similarity to any other $P$. aeruginosa proteins.

the increased expression of $l p t D$ must be bypassing some other mutation. Our preliminary studies showed that the mucoid phenotype in sap22 with a mutation in algO (PA3257) can be rescued by another cosmid (Reiling et al., 2005; Sautter et al., 2012). This led to our hypothesis that sap27 may harbor a mutation in $\operatorname{algO}(P A 3257)$. The introduction of a 
plasmid carrying algO (pAlgO) into sap27 rescued the mucoid phenotype (Figure 2.3(d) and Figure 2.4). Ideally, the cosmid containing $\operatorname{alg} O$ should have complemented sap27 in the original en masse screening. However, it was missed in-part due to the subjective nature of the procedure. The sequence analysis of algO, encoding a 709-amino acid long protein in sap27 genome, revealed an insertion of a single nucleotide, thymine (T), at position 3,643,250 on the genome (Figure 2.6). This insertion results in a frame-shift mutation leading to premature termination of $\operatorname{algO}$ at codon 320 (Fig. 2.6), which is converted from glutamic acid to UGA, an opal stop codon. The truncated protein has an identity to AlgO at the first 106 amino acids of the $\mathrm{N}$-terminus and the remaining 213 amino acids share no similarity to any other $P$. aeruginosa proteins. In fact, the same mutation was identified in sap22 (Reiling et al., 2005; Sautter et al., 2012). However, the codon number was stated differently as the start codon for $\operatorname{alg} O$ is off by 11 codons that were recently corrected on pseudomonas.com (Winsor et al., 2016).

To ensure the phenotype seen in sap27 is not strain-specific and can be reproduced in sap22, the plasmid $\mathrm{pLptD}$ was introduced in the latter rescuing the mucoid phenotype (Figure 2.3(c)). To further confirm that $l p t D$ in trans can overcome the algO mutation, a clean in-frame deletion of $\operatorname{alg} O$ was constructed in PAO1 and PDO300 and the strains were named as PAO $\triangle$ alg $O\left(\mathrm{Alg}^{-}\right)$and $\mathrm{PDO} \Delta a \lg O\left(\mathrm{Alg}^{-}\right)$, respectively. The plasmid pLptDSurA-LptE was introduced in these strains and induced with arabinose. The increase in lptD copy number (expected to be between 1-7 copies) (Jahn et al., 2016). leading to higher protein levels in PAO1 and $\mathrm{PAO} \Delta$ algO results in the strains turning mucoid with significant production of alginate ((Figure 2.3(c), Figure 2.4). Thus, the LptD-dependent alginate-production does not require $\operatorname{alg} O$. The induction of $\operatorname{lptD}$ in PDO $\triangle a l g O\left(\mathrm{Alg}^{-}\right)$ 
failed to rescue the alginate phenotype (Figure 2.4). However, the alginate phenotype can be rescued by $l p t D$ in the sap27 mutant strain (Figure 2.4). Both of these strains express truncated MucA (MucA22). The difference between PDO $\triangle$ algO and sap27 (PDOalgO107) is that one has no $\mathrm{AlgO}$ whereas the latter expresses a truncated $\mathrm{AlgO}$. This suggests that LptD-dependent alginate production in sap27 requires the truncated $\mathrm{AlgO}$. One cannot rule out the possibility that the truncated $\mathrm{AlgO}$ in sap27 might be contributing to the observed phenotype.

\section{Increased expression of lptD-surA-lptE in $\triangle a l g W$ mutant of PAO1 and PDO300}

To determine if LptD-dependent induction of alginate production has a role in the RIP cascade, attempts were made to construct deletion mutants of algW and mисP. Repeated efforts to make тисP deletion mutants failed. However, PAO1 and PDO300 derivatives with clean in-frame $\operatorname{alg} W$ deletions were constructed and are referred to as PAO $\Delta a \lg W$ and PDO $\Delta a \lg W$, respectively. As expected, the loss of $\operatorname{alg} W$ resulted in the loss of alginate production in PDO300 (Figure 2.4). Multiple efforts to complement PDO $\Delta a \lg W$ with $\operatorname{alg} W$ also failed. The plasmids $\mathrm{pLptD}$ and the pLptD-SurA-LptE were introduced into $\mathrm{PAO} \Delta a \lg W$ and $\mathrm{PDO} \Delta a \lg W$. The mucoid phenotype of PDO $\Delta a \lg W$ was rescued by increasing the copy number of $\operatorname{lptD}$ (Figure 2.4). Inducing the expression of $l p t D$ in $\mathrm{PAO} \Delta a l g W$ did not result in alginate production (Figure 2.4). This suggests that the LptD-dependent alginate production in PAO1 requires AlgW. Thus, in a mucA22 background, LptD can bypass AlgW. 


\section{Discussion}

The emergence of alginate-producing strains of $P$. aeruginosa in patients with CF is an indication of chronic infection that signals a deterioration of lung function and ultimately pulmonary failure. The importance of alginate in $\mathrm{CF}$ patients was corroborated in the early 1970s. However, the genetic determinant responsible for the conversion was mapped to a single locus in the 1980s, and the genes responsible were identified in the 1990s. Almost 35 years later, with the identification of over 30 alginate genes, a solution to prevent mucoid conversion continues to elude us. This study was undertaken to identify novel genes by mapping second site-suppressor mutations of alginate production. The gainof-phenotype in a non-mucoid sap27 was mapped to lptD (PA0595) encoding an outer membrane protein. To date, all the genes involved in the regulation of alginate production encode for products that reside in the periplasm, the inner membrane, and the cytoplasm. This report demonstrates the potential role of a $P$. aeruginosa OMP, LptD (PA0595), in alginate production.

LptD transports lipopolysaccharide to the outer leaflet of the outer membrane and is an essential OMP in E. coli and P. aeruginosa but not in Neisseria meningitidis (Bos et al., 2004; Braun and Silhavy, 2002; Werneburg et al., 2012). In E. coli, LptD is a member of the $\sigma^{\mathrm{E}}$ regulon with multiple designations: $\mathrm{LptD}$, OstA, and Imp owing to its different roles (Abe et al., 2003; Aono et al., 1994; Braun and Silhavy, 2002; Dartigalongue et al., 2001; Sampson et al., 1989). Actually, LptD designation comes from LipoPolysaccharide Transport Protein because of its involvement in outer membrane biogenesis (Braun and Silhavy, 2002; Sperandeo et al., 2009), the OstA from organic solvent tolerance A because of its importance in the growth of $E$. coli $\mathrm{K} 12$ in xylene and n-hexane (Abe et al., 2003; 
Aono et al., 1994); and finally, Imp for $\boldsymbol{i n c r e a s e d} \boldsymbol{m}$ embrane $\boldsymbol{p}$ ermeability that contributes to resistance to antibiotics, detergents and dyes (Charlson et al., 2006; Sampson et al., 1989). P. aeruginosa LptD is also involved in LPS transport and controlling membrane permeability and confers resistance to antibiotics such as rifampin, ciprofloxacin, tetracycline, novobiocin, azithromycin and imipenem (Balibar and Grabowicz, 2016; Werneburg et al., 2012). Similar to E. coli, P. aeruginosa LptD is involved in LPS palmitoylation (Balibar and Grabowicz, 2016; Ruiz et al., 2008; Wu et al., 2006). It is not uncommon to find proteins with multiple roles in bacterial physiology, for an example, AlgC is involved in LPS, Pel, Psl, alginate, and rhamnolipid production (Ma et al., 2012; Olvera et al., 1999; Zielinski et al., 1991).

\section{Outer membrane protein LptD regulates alginate production}

One can argue that increasing expression will lead to misfolding of LptD that may cause alginate production in sap27, since it requires LptE and the SurA as chaperone (Chimalakonda et al., 2011; Lazar et al., 1998). However, the co-expression of $l p t D$ with surA and lptE continued to restore the mucoid phenotype of sap27 ruling out misfolding of LptD (Figure 2.4). Further expression of optP encoding an OMP of comparable size in sap27 did not result in rescuing the mucoid phenotype (Figure 2.4). These results certainly argue that LptD, an OMP, may have a specific role in the regulation of alginate production.

\section{LptD bypasses AlgO107}

It is interesting that the sap27 strain did not have any mutations in the lptD locus but in algO. A role for $\mathrm{LptD}$ in alginate production is further augmented by its ability to rescue the loss of $\operatorname{alg} O$ in $\operatorname{sap} 22$ that was previously identified with the same mutation 
(Figure 2.4). In a previous publication, the allele was named as algO96 (Sautter et al., 2012). However, with correction of the ATG start codon location upstream, we renamed the allele as algO107. Increasing the expression of $\operatorname{lptD}$ bypassed algO107 but failed to rescue the alginate phenotype when algO was completely deleted in PDO300. This suggests that the presence of truncated AlgO contributes to the observed phenotype. This phenotype is independent of the proteolytic activity of $\mathrm{AlgO}$ as the truncated protein (AlgO107) is missing that catalytic triad Ser490, Asp501, and Lys515. It is not possible to rule out that the last 213-amino acid in the AlgO107 is contributing to the observed phenotype without further experimentation. Previously, it was argued that MucA22 is the substrate for $\mathrm{AlgO}$, a putative serine protease (Reiling et al., 2005). However, no concrete evidence has surfaced to support this argument.

\section{LptD influences members of RIP cascade}

To determine if LptD played a role in the RIP cascade, algW was deleted in PDO300 and PAO1. The loss of alginate production in PDO $\Delta$ algW was rescued by increasing $\operatorname{lptD}$ expression (Figure 2.4). This observation suggests that the release of AlgT/U by MucA22 (Figure 1.7 (B)) does not require site-1 cleavage by AlgW, despite the fact that the major $\mathrm{AlgW}$ cleavage site on MucA $\left(\mathrm{A}_{136}\right.$ and $\left.\mathrm{G}_{137}\right)$ is still present in MucA22 (Damron and $\mathrm{Yu}, 2011$ ). However, in the wild-type $P$. aeruginosa PAO1, mucoid conversion by overexpression of $\operatorname{lptD}$ requires $\mathrm{AlgW}$. This finding argues that $\mathrm{LptD}$ directly or indirectly influences the members of RIP cascade. 


\section{Two pathways for MucA proteolysis}

As previously shown, our results also suggest a difference in the regulation of alginate production in wild-type versus isolates with the mucA22 allele (Qiu et al., 2007). In the wild-type background, alginate production is very tightly controlled since MucA is intact and protected by MucB against proteolytic cleavage (Cezairliyan and Sauer, 2009). We propose that, upon increased expression of $\operatorname{lptD}, \mathrm{Alg} \mathrm{W}$ is activated to initiate MucA



Figure 2.7. Model for mucoid conversion due to increase expression of $\operatorname{lptD}$ in $m u c A^{+}$ (PAO1) and $\boldsymbol{m u c A 2 2}$ (PDO300) backgrounds. We proposed that (a) in PAO1, increased expression of lptD displaces $\mathrm{MucB}$ and induces $\mathrm{AlgW}$ to cleave MucA resulting in proteolysis of MucA as described previously in figure 1.7. There might not be any role for $\mathrm{AlgO}$ in PAO1 under the tested conditions. (b) in the mucA22 background, MucB cannot bind to MucA, thus the cleavage site of MucA22 is exposed. The overexpression of LptD might activate the truncated $\mathrm{AlgO}$ leading to MucA22 proteolysis via an unknown mechanism. 
cleavage followed by MucP to release AlgT/U for alginate synthesis (Figure 2.7(a)). In the mucoid $P$. aeruginosa isolates with the mucA22 allele, $\mathrm{AlgO}$ seems to be playing an important role by activating cleavage of the truncated MucA22 (Figure 2.7(b)) either directly or indirectly by means of some unknown protein. Mechanistically, the specific role of $\mathrm{LptD}$ in the activation of $\mathrm{AlgW}$ or $\mathrm{AlgO}$ is not clear at the moment. The regulation of alginate production due to LptD seems depend on strain backgrounds and requires further investigation.

The X-ray crystal structure of $E$. coli LptD shows the protein has C-terminal $\beta$ barrel domain while its $\mathrm{N}$-terminal periplasmic domain has a jelly-roll like structure (Dong et al., 2014; Qiao et al., 2014). Bioinformatic analysis shows LptD of P. aeruginosa is structurally similar to that of E. coli (Werneburg et al., 2012). It is not known whether the $\mathrm{N}$-terminal domain of $\mathrm{LtpD}$ could interact with other periplasmic proteins to induce alginate production. Moreover, it is possible that the N-terminal domain of LptD or LPS might displace MucB, leaving MucA vulnerable to proteolysis (Figure 2.7(b)) as seen in the E. coli counterpart (Lima et al., 2013; Wilken et al., 2004; Wollmann and Zeth, 2007). To understand the mechanistic detail standard protein-protein interaction studies (pulldown, cross-linking, competition assays) can be performed to explore if there is any direct interaction between LptD and MucB and/or MucA.

\section{Implication}

To date, $P$. aeruginosa $\mathrm{LptD}$ has not been associated with alginate production. $P$. aeruginosa $\mathrm{LptD}$ is a target of peptidomimetic antibiotics based on the antimicrobial peptide protegrin I that appears to be species-specific (Srinivas et al., 2010; Werneburg et 
al., 2012). It is also shown that depletion of LptD results in antibiotic susceptibility in $P$. aeruginosa and Acinetobacter baumannii (Balibar and Grabowicz, 2016; Bojkovic et al., 2015). Furthermore, $L p t D$ is a subject of study for a candidate vaccine in Gram-negative bacteria such as Neisseria gonorrhea and Vibrio parahaemolyticus (Zha et al., 2016; Zielke et al., 2016) Multidimensional protein identification technology showed that in $P$. aeruginosa 63 proteins including LptD are exposed to the extracellular environment (Srinivas et al., 2010; Vecchietti et al., 2012). Thus, it would be interesting to determine if the peptidomimetic antibiotics, as well as a vaccine against $\mathrm{LptD}$, can prevent alginate production in $P$. aeruginosa. 


\section{CHAPTER 3}

Pyridoxal 5'-phosphate (PLP) enhances pyocyanin biosynthesis in Pseudomonas aeruginosa 


\begin{abstract}
Pseudomonas aeruginosa is a metabolically versatile pathogen. In previous chapter (Chapter 2), we identified an operon that contains three genes $\operatorname{lpt} D, \operatorname{surA}$ and $p d x A$. The $p d x A$ encoding an outer membrane protein, chaperone protein, and an enzyme involved in pyridoxal 5'-phosphate (PLP) biosynthesis, respectively. PLP is also known as vitamin B6. PLP biosynthetic pathway has a common precursor, D-erythrose-4-phosphate, that converted to chorismic acid that can either synthesize pyocyanin and aromatic amino acids. We hypothesized that PLP is involved in P. aeruginosa virulence. Culturing bacteria in medium supplemented with PLP resulted in increased pyocyanin production. Among the aromatic amino acids, tryptophan, phenylalanine and tyrosine the latter two shunted the pathway to produce pyocyanin. The finding in this chapter show that PLP, and aromatic amino acids phenylalanine and tyrosine indeed causes $P$. aeruginosa to increase in pyocyanin production suggesting a possible role for $p d x A$ in virulence.
\end{abstract}

\title{
Introduction
}

P. aeruginosa, a Gram-negative bacterium, is a leading cause of infection in cystic fibrosis (CF) patients (CFF, 2016). P. aeruginosa is a metabolically versatile organism which in part is due to pyridoxal 5'-phosphate (PLP) which is only the active $\mathrm{B}_{6}$ vitamers. PLP serves as a cofactor of various essential enzymes that are involved in the metabolism of amino acids, fatty acids, and carbohydrates (Percudani and Peracchi, 2003; Percudani and Peracchi, 2009). Bioinformatic analyses show 23 proteins encoded by $P$. aeruginosa require PLP as a co-factor (Table 3.1) (Percudani and Peracchi, 2009). 
There are two biochemical pathways for PLP biosynthesis, and these pathways are mutually exclusive (Fitzpatrick et al., 2007). In P. aeruginosa, the PLP is synthesized by a deoxyxylulose-5-phosphate (DXP) dependent pathway (Fitzpatrick et al., 2007). The canonical PLP binding sequence is Val-Ile-Tyr-Glu-Thr-Glu-Ser-Thr-His-Lys-Leu-LeuAla-Ala-Phe where either histidine or lysine residue may play a direct role in catalysis

\section{Table 3.1. Predicted ORFs that requires PLP as a co-factor}

\begin{tabular}{cccl} 
S/N & Gene & Locus & \multicolumn{1}{c}{ Protein } \\
1 & cysM & PA0932 & Cysteine synthase \\
2 & & PA3659 & Probable aminotransferase \\
3 & bauA & PA1232 & Beta-alanine-pyruvate transaminase \\
4 & $p h n W$ & PA1310 & 2-aminoethylphosphonate:Pyruvate transferase \\
5 & $p h h C$ & PA0870 & Aromatic amino acid aminotransferase \\
6 & $p v d H$ & PA2413 & Diaminobutyric acid aminotransferase \\
7 & aruC & PA0895 & N2-Succinylornithine 5-aminotransferase \\
8 & aruH & PA4976 & Arginine:Pyruvate transaminase \\
9 & selA & PA4808 & L-seryl-tRNA(Ser) selenium transferase \\
10 & ldcA & PA1818 & Probable Orn/Arg/Lys decarboxylase \\
11 & lysA & PA5277 & Diaminopimelate decarboxylase \\
12 & itaA & PA5413 & Low-specificity L-threonine aldolase \\
13 & pabC & PA2964 & 4-amino-4-deoxychorismate lyase \\
14 & trpB & PA0036 & Tryptophan synthase beta chain \\
15 & thrC & PA3735 & Threonine synthase \\
16 & & PA2683 & Probable serine/threonine dehydratase \\
17 & & PA3357 & D-serine dehydratase \\
18 & & PA0400 & probable cystathionine gamma-lyase \\
19 & alr & PA4930 & Alanine racemase, biosynthetic \\
20 & hemL & PA3977 & Glutamate-1-semialdeyde 2,1-aminomutase \\
21 & arnB & PA3552 & UDS-4-deoxy-L-arabinose synthase \\
22 & cobC & PA1276 & Cobalamin biosynthetic protein CobC \\
23 & metZ & PA3107 & O-succinylhomoserine sulfhydrylase
\end{tabular}


(Sabo and Fischer, 1974; Wolosker et al., 1999). The role of the PLP in the production of one of the virulence factors, pyocyanin, has not been established yet in P. aeruginosa.

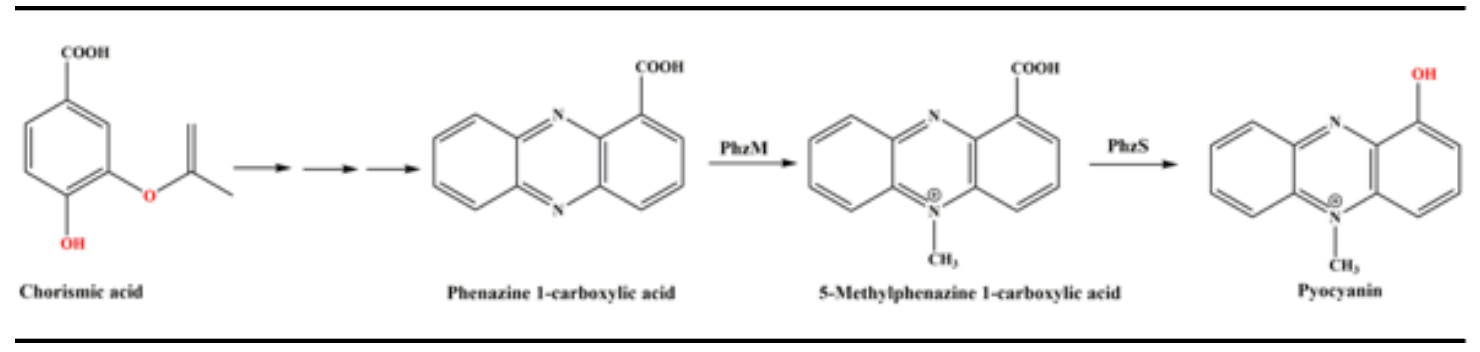

Figure 3.1. Synthesis of pyocyanin from chorismic acid. Chroismic acid undergoes biosynthetic reaction to synthesize phenazine-1-carboxylic which is then by enzyme PhzM converted to 5-Methylphenazine-1-carboxylic to synthesize pyocyanin by PhzS. Courtesy (Rada and Leto, 2013)

Pyocyanin is a water-soluble, blue-green phenazine compound, and functions as an important virulence factor, as it affects $P$. aeruginosa biofilm development as well as the release of extracellular DNA (Baron and Rowe, 1981; Das et al., 2015; Das and Manefield, 2012; Frank and Demoss, 1959; Grossowicz et al., 1957). In P. aeruginosa, one of the precursor molecules of pyocyanin is chorismic acid (Figure 3.1). Chorismic acid is also involved in the synthesis of aromatic acid (Palmer et al., 2013; Tamir and Srinivasan, 1971). The function of pyocyanin is to balance the $\mathrm{NADH} / \mathrm{NAD}^{+}$ratio in the $P$. aeruginosa cytoplasm when the microbes are in stationary phase (Price-Whelan et al., 2007).

Pyocyanin has been shown to have antibacterial activity against Gram-positive bacteria Micrococcus luteus, Gram-negative bacteria E. coli, and Shewanella oneidensis (Costa et al., 2015; Kanthakumar et al., 1993; Mazzola et al., 1992). Pyocyanin biosynthesis is increased when $P$. aeruginosa is co-cultured with Candida albicans and was shown to be toxic to the fungus (Gibson et al., 2009; Morales et al., 2010; Trejo- 
Hernandez et al., 2014). By using Saccharomyces cerevisiae and the human lung carcinoma alveolar type II cell line A549, it was shown that pyocyanin generated reactive oxygen species that inactivated human vacuolar ATPase, negatively impacting the lung function of people with CF (Ran et al., 2003). Additionally, pyocyanin has been shown to cause erythoptosis-inducing effects in erythrocytes suggesting a systemic effect on patients suffering from CF (Qadri et al., 2016). In a mouse model, it has been shown that pyocyanin caused tissue damage and necrosis that contributed to the development of pneumonia (Lau et al., 2004).

P. aeruginosa harbors two homologous core loci (phzA1-G1 and phzA2-G2) and three additional genes $(p h z M, p h z S$, and $p h z H)$ that are involved in the pyocyanin biosynthesis (Figure 3.1)(Mavrodi et al., 2001). None of these genes appear to have a canonical PLP-binding site. However, both PLP and pyocyanin synthesis use the same starting material, D-erythrose-4-phosphate (Figure 3.2). What drives the reaction toward pyocyanin production is unclear. Growing $P$. aeruginosa in a synthetic CF sputum medium supplemented with the aromatic amino acids also enhanced the production of pyocyanin (Palmer et al., 2007). It was shown that Pseudomonas quinolone signal (PQS), a cell-cell signaling system, positively affects pyocyanin production.

Recently, we demonstrated that the expression of lptD restores the mucoid phenotype in a nonmucoid strain (Pandey et al., 2018). The lptD gene is part of a threegene-operon $l p t D$-surA-pdxA. The $\operatorname{lptD}$ gene encodes an outer membrane protein (OMP) involved in lipopolysaccharide transport (Braun and Silhavy, 2002; Sperandeo et al., 2009; Wu et al., 2006). SurA influences the biogenesis of LptD whereas PdxA is involved in pyridoxal 5'-phosphate synthesis (PLP) (Figure 3.2)(Denoncin et al., 2010; Vertommen et 
$a l ., 2009)$. The PLP is one of the six B 6 vitamers that includes pyridoxal, pyridoxine, and pyridoxamine and their phosphorylated derivatives PLP, pyridoxine-5-phosphate, and pyrdidoxamine-5-phosphate (Figure 3.3). PLP is the only active form of vitamin $\mathrm{B}_{6}$ that is serves as cofactor for more than 160 vitamin $\mathrm{B}_{6}$-dependent enzymes (Percudani and Peracchi, 2003). The vitamin $\mathrm{B}_{6}$ dependent enzymes are involved in amino acid metabolism, amine biosynthesis, carbohydrate breakdown, heme synthesis, nucleic acid synthesis and, neurotransmitter biosynthesis (Percudani and Peracchi, 2009). Animals cannot synthesize PLP but have to depend on microorganisms and plants (Fitzpatrick et $a l .$, 2007). The focus of this chapter is PdxA, as it has not been previously characterized. Quorum sensing genes also regulate pyocyanin biosynthesis in P. aeruginosa (Lee and Zhang, 2015). Many of these genes are involved in amino acid syntheses. The role of these genes in pyocyanin production, however, has never been explored. One of the intermediates in the pathway, chorismic acid, can either be funneled into the synthesis of aromatic amino acids or pyocyanin (Figure 3.2).

It is known that the presence of exogenous aromatic amino acids also results in pyocyanin production (Palmer et al., 2007). The present study initiated the work needed to tease apart the intricate mechanism that drives pyocyanin production and possible relationship with PLP. 




Figure 3.2. Detail pathway for the synthesis of pyocyanin. The pathway to synthesize aromatic amino acid, pyocyanin and pyridoxal 5'-phosphate is shown. The solid line and dotted line indicate single and multi-step pathways, respectively. 


\section{Methods}

\section{Bacterial strains, media and growth conditions}

The bacterial strain used in this study is $P$. aeruginosa PAO1 strain. It was cultured overnight at $37^{\circ} \mathrm{C}$. Then, the next day it was sub-cultured in a flask until an $\mathrm{OD}_{600}=1$. Then, the culture was diluted to an $\mathrm{OD}_{600}=0.5$ in fresh $\mathrm{LB}$. Then, a $2.5-\mathrm{ml}$ of the diluted culture was taken and supplemented with aromatic amino acids or PLP to the desired concentration to a final volume of 5-ml and the mixture was cultured was incubated for 15 hours.

\section{Pyocyanin assay}

The pyocyanin assay was carried out by extracting the pigment from culture supernatant using the chloroform-HCL technique as previously described (Essar et al., 1990).<smiles>[C-]c1ncc(C(C)([O])F)c(CO)c1O</smiles>

Pyridoxine

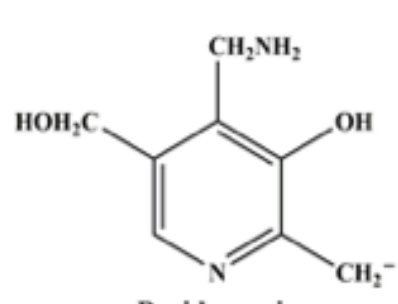

Pyridoxamine<smiles>[CH2-]c1ncc(CO)c(C=O)c1O</smiles>

Pyridoxal<smiles>Cc1ncc(C(=O)[O-])c(C(=O)[O-])c1O</smiles>

Pyridoxine 5'-phosphate<smiles>CCCCCCc1c(C(=O)[O-])cnc(C)c1O</smiles>

Pyridoxamine 5'-phosphate<smiles></smiles>

Pyridoxal 5'-phosphate

Figure 3.3. Different forms of $\mathbf{B}_{6}$ vitamers. $B_{6}$ vitamers are pyridoxine, pyridoxamine, pyridoxal and their phosphate forms. Pyridoxal 5'-phosphate is active B6 vitamers. Courtesy (Vrolijk et al., 2017). 
A 5-ml culture was centrifuged at $8500 \mathrm{rpm}$ for $10 \mathrm{~min}$ at $18{ }^{\circ} \mathrm{C}$, then $4-\mathrm{ml}$ of the supernatant was mixed with 3-ml of chloroform, then the mixture was vortexed for 2 minutes and the phases were allowed to separate settled down for $20 \mathrm{~min}$. Then, a 2-ml of the lower phase was aspirated and mixed with $1-\mathrm{ml}$ of $0.2 \mathrm{~N} \mathrm{HCl}$, followed by vortexing for $2 \mathrm{~min}$ and let it stand for $20 \mathrm{~min}$. Then, the top layer was used to quantify the pyocyanin by measuring at the absorbance at $520 \mathrm{~nm}\left(\mathrm{~A}_{520}\right)$.

\section{RNA isolation, cDNA synthesis, and qPCR}

All primers used in the experiments were synthesized and supplied by Integrated DNA Technologies (Coralville, IA) (Table 3.2).

\begin{tabular}{ccc}
\multicolumn{3}{c}{ Table 3.2. Primers used for qPCR } \\
S/N & Primer name & Primers \\
1 & SP_qRT_phzC1 (F) & GGCCGGTGATCCGCGTCG \\
2 & SP_qRT_phzC1(R) & CCGCCGCATAGCCCTTGAG \\
3 & SP_qRT_phzE1(F) & ATGACCTGCTGGCGCTGAT \\
4 & SP_qRT_phzE1(R) & GCGACCGATCTCGTCGGCG \\
5 & DBS_qRT_clpX(F) & TGCGATTACGATGTGGAGA \\
6 & DBS_qRT_clpX(R) & CCCTCGATGAGCTTCAGCA
\end{tabular}

The Ribonucleic acid (RNA) was isolated from the prototypic $P$. aeruginosa grown in LB broth. Briefly, the overnight culture of $P$. aeruginosa grown in LB broth at $37{ }^{\circ} \mathrm{C}$ was washed with sterile $0.85 \%$ saline solution to remove spent media and was sub-cultured at $37{ }^{\circ} \mathrm{C}, 200 \mathrm{rpm}$ in LB media. When the cells reached an optical density of $600 \mathrm{~nm}$ $\left(\mathrm{OD}_{600}\right)$ of 0.6 - 0.7 the bacterial culture was treated with $5.92 \mathrm{mM}$ PLP for 1 hour. Posttreatment, RNA was stabilized by addition of phenol-ethanol mixture. The stabilized RNA was then isolated using RNeasy Mini Kit (Qiagen, Inc Venio, Limburg, Netherland) as per 
the manufacturer's protocol. Residual genomic DNA contamination was removed using RQ1 RNase-free DNase (Promega, Madison, Wl, USA) and RNA was re-purified using Rneasy kit (Qiagen, Inc Venio, Limburg, Netherlands). The quality of purified RNA was assessed on a denaturing agarose gel (NorthernMax Gly, Ambion, Life Technologies, Carlsbad, CA, USA) and quantified at $260 \mathrm{~nm}$ (BioTEK, Synergy HT, Winooski, VT, USA). The complementary DNA (cDNA) was then synthesized by annealing NS5 random primers to total purified RNA and subsequent extension was carried out using SuperScript 111 reverse transcriptase (Invitrogen, Life Technologies, Carlsbad, CA, USA). The quantitative polymerase chain reaction (qPCR) to study expression levels of $p h z C 1$ and phzE1 under PLP induction was performed using an Applied Biosystem Step One cycler and detection system with PowerSYBR Green PCR MasterMix with ROX (Applied Biosystems, Life Technologies, Carlsbad, CA, USA). The cycling conditions used were 95 ${ }^{\circ} \mathrm{C}$ (2 minutes, holding); 40 cycles of $95{ }^{\circ} \mathrm{C}$ (15 sec), $60{ }^{\circ} \mathrm{C}(60$ seconds, cycling $) ; 95{ }^{\circ} \mathrm{C}$ (15 second), $60{ }^{\circ} \mathrm{C}\left(1\right.$ minute), $95{ }^{\circ} \mathrm{C}(15$ second $)\left(0.6{ }^{\circ} \mathrm{C}\right.$ ramp) (melt curve). Expression was normalized to the housekeeping gene $\operatorname{clp} X$ (PA1802), whose expression remained unchanged under the tested conditions.

\section{Results}

\section{PLP up-regulates the pyocyanin production}

To investigate the role of PLP in pyocyanin biosynthesis, $P$. aeruginosa was cultured for 15 hours in LB with and without PLP. The assay showed that $P$. aeruginosa produced a significantly higher quantity of pyocyanin in the presence of PLP suggesting that PLP influences a role in pyocyanin biosynthesis $P$. aeruginosa (Figure. 3.4). 


\section{Pyocyanin production in the presence of aromatic amino acids}

Common precursor molecules, chorismite and anthranilate are used to synthesize pyocyanin, pseudomonas quinolone signal, and aromatic amino acids (Figure 3.3) (Lee and Zhang, 2015). So, we hypothesized that supplementation of aromatic amino acids in LB medium would divert intracellular chorismate to pyocyanin synthesis. To test this hypothesis, $P$. aeruginosa was grown in LB medium supplemented with different concentrations of phenylalanine, tyrosine, and tryptophan. There was no significant change in pyocyanin production when $P$. aeruginosa was grown in the LB medium supplemented with tryptophan (Figure 3.4). Pyocyanin was significantly overproduced when $P$. aeruginosa was grown in LB supplemented with phenylalanine and tyrosine (Figure 3.4).

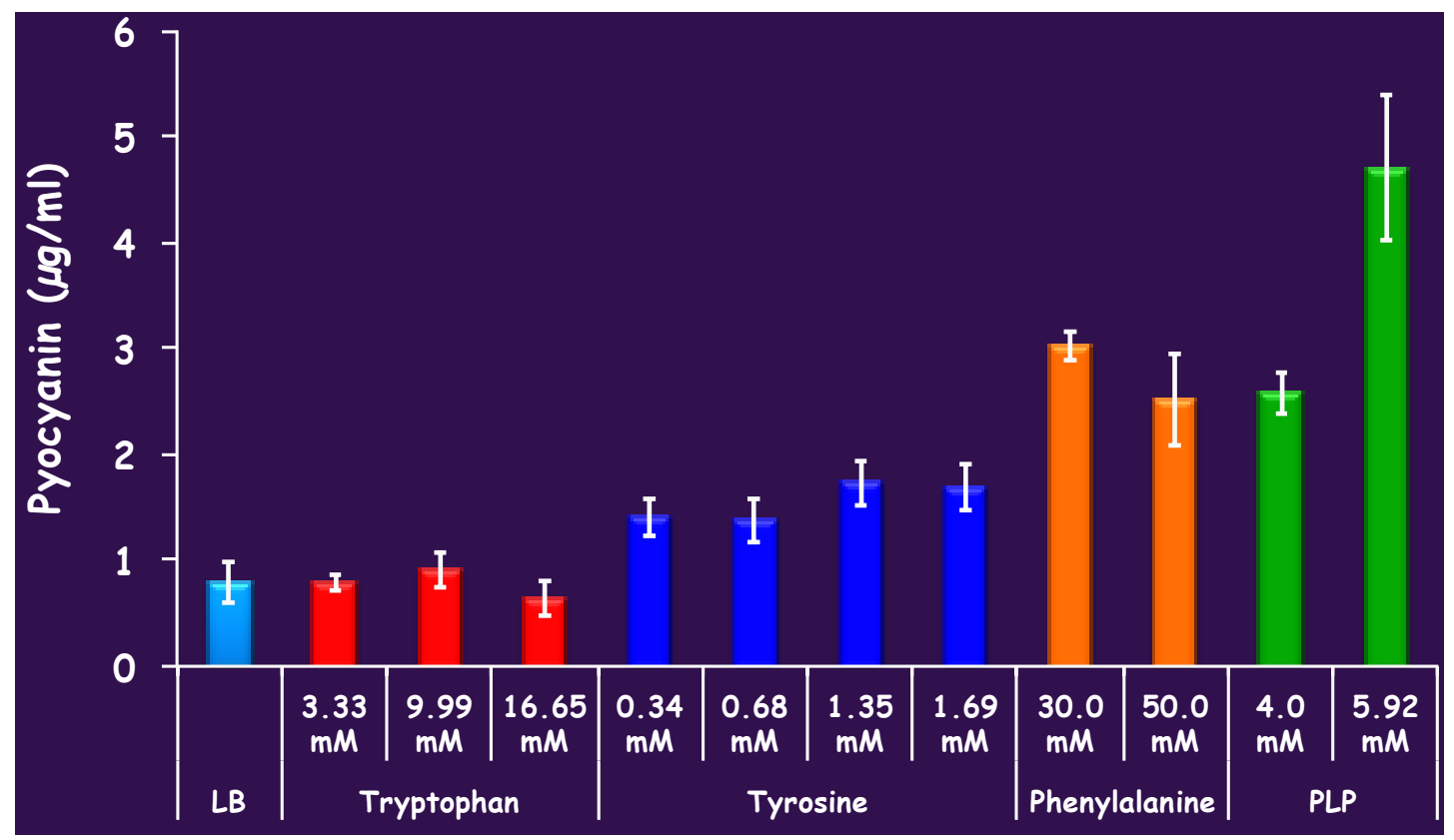

Figure 3.4. Pyocyanin production in the presence of PLP and aromatic amino acids. P. aeruginosa PAO1 was grown in aromatic amino acids or PLP and levels of pyocyanin were quantified. 


\section{PLP increases the transcription of the pyocyanin biosynthesis operon}

In $P$. aeruginosa, two operons are involved in pyocyanin biosynthesis. The pyocyanin biosynthesis operon phzl (phzA1B1C1D1E1G1) is located at positions $4,713,795$ to $4,720,062$ bp in the genome, while the other operon, phz2 (phzA2B2C2D2E2G2), is located approximately $2.6 \mathrm{Mb}$ from phzl at the position 2,070,685 to 2,076,985 bp (Winsor et al., 2016). One operon is required for pyocyanin biosynthesis (Cui et al., 2016; Recinos et al., 2012).

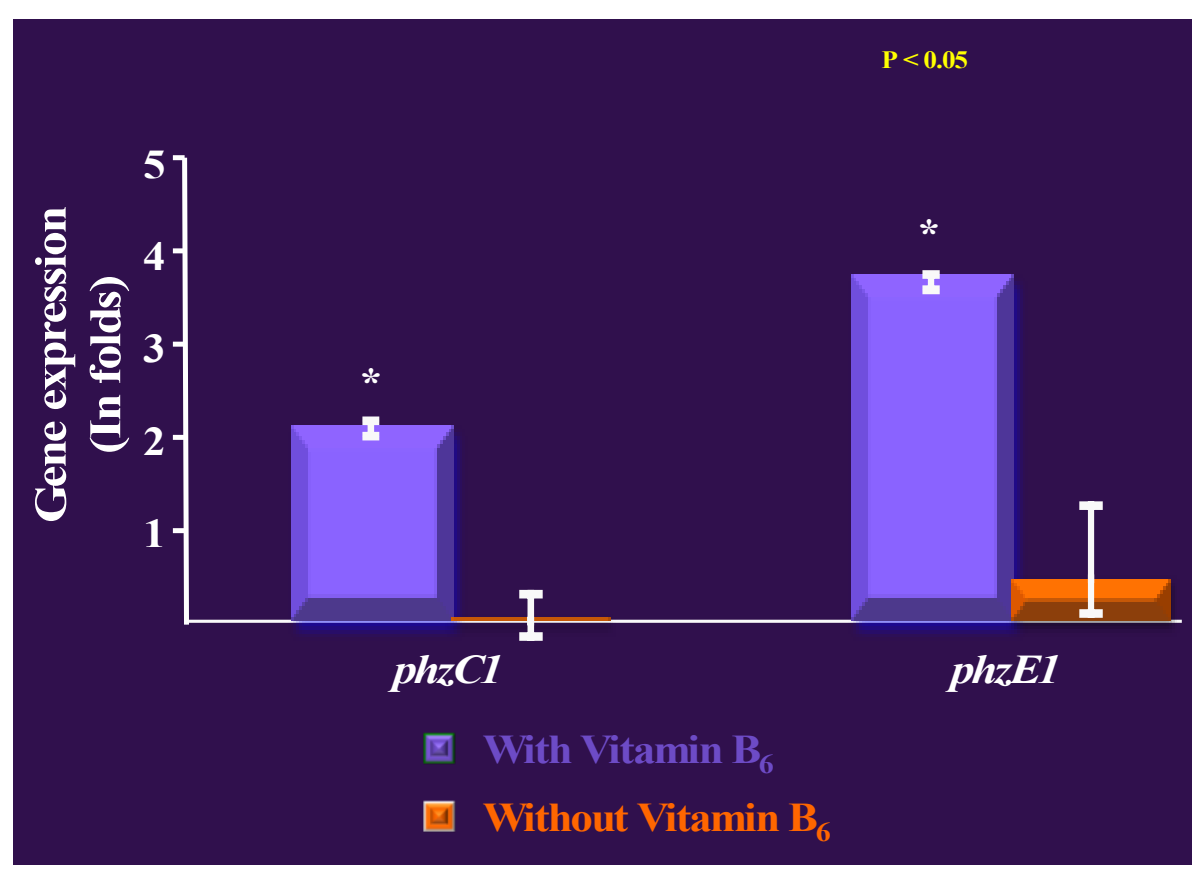

Figure 3.5. Expression of genes in phz-operon due to PLP. P. aruginosa PAO1 was grown in LB media supplemented with or without vitamin B6. Then RNA was isolated, and cDNA was synthesized. The qPCR was done to quantify the expression level phzCl and $p h z E 1$.

To identify whether PLP affects phenazine genes at the operons at the transcriptional level, we determined the relative levels of phzC1 (PA4212) and phzE1 
(PA4214) transcripts by qPCR. P. aeruginosa PAO1 was grown in LB media supplemented with/without PLP. Complementary DNA (cDNA) was synthesized and qPCR was performed using the primers shown in (Table 3.2). Our result showed that $p h z C l$ and $p h z E l$ transcripts increased three and five-fold, respectively, when $P$. aeruginosa was grown in the PLP-treated versus untreated media (Figure 3.5). Our results indicate that PLP increases the transcription of the phzl operon. However, whether PLP directly or indirectly plays a role in the increased transcription of phzl operon remain to be explored.

\section{Discussion}

Little is known about the role of PLP biosynthesis and its role in the virulence of bacteria. Neither the biosynthesis pathway in $P$. aeruginosa nor the importance of PLP in virulence has been explored. In this chapter, the role of PLP, as well as preliminary mechanism of pyocyanin biosynthesis when the $P$. aeruginosa is grown in medium supplemented with PLP, is explored.

PLP is synthesized in bacteria via two de novo pathways known as deoxyxylulose5-phosphate (DXP)-dependent and DXP-independent pathway (Fitzpatrick et al., 2007). The DXP-dependent pathway has been mostly present in $\gamma$ proteobacteria such as $P$. aeruginosa where $p d x A$ is involved in synthesis of 2-amino-3-oxo-4-phosphoxoybutanoate from 4-phospho-hydroxy-L-threonine (Figure 3.2). Our results showed that the growth of P. aeruginosa in the presence of PLP increased the production of pyocyanin production, one of the virulence factors of $P$. aeruginosa. The pyocyanin and PLP are synthesized from a common precursor D-erythrose -4-phosphate (Figure 3.2). From D-erythrose-4phosphate either PLP or chorismic acid is synthesized via two independent biosynthetic 
pathways. The chorismic acid further can be used to synthesize either aromatic amino acids or pyocyanin (Figure 3.2). We hypothesized that culturing of $P$. aeruginosa in presence of PLP shunted D-erythrose-4-phosphate to a pathway in favor of pyocyanin production. Furthermore, since chorismic acid is used synthesis of either aromatic amino acid or pyocyanin production, we hypothesized that in excess of aromatic amino acids, chorismic acid would push the pathway to the production of pyocyanin. Our results suggest indeed growth of $P$. aeruginosa in a media supplemented with aromatic amino acids tyrosine and phenylalanine increased the production of pyocyanin.

Our data show that among the three aromatic amino acids the highest amount of pyocyanin was produced when $P$. aeruginosa was cultured in LB medium supplemented with excess phenylalanine whereas lowest in the presence of tryptophan. The higher amount of pyocyanin biosynthesis when $P$. aeruginosa was grown in presence of phenylalanine may be the result of the existence of the aromatic amino acid permease transport systems I and II (Kay and Gronlund, 1971). The aromatic amino acid permease transport system transport systems have different affinities for the three aromatic amino acids, system I recognizes and transports phenylalanine, tyrosine, and tryptophan respectively in this order, whereas the aromatic transport system II recognizes tryptophan, phenylalanine, and tyrosine in this order (Kay and Gronlund, 1971). In addition, it has been shown that phenylalanine and tyrosine accumulates inside $P$. aeruginosa to a concentration approximately 10 times greater than that found for tryptophan (Kay and Gronlund, 1971). 


\section{Future direction}

The results still leave the role of $p d x A$ unanswered. The only way to show $p d x A$ role in virulence and vitamin $\mathrm{B}_{6}$ synthesis is by constructing knockout strain. However, attempts to delete the gene failed suggesting that it is an essential gene, as such a conditional mutant need to be constructed.

Construction of a conditional $p d x A$ mutant

A strain with the $p d x A$ gene under the control of the $\operatorname{araBAD}$ promoter $\left(\mathrm{P}_{\mathrm{BAD}}\right)$ should be constructed. A 300-bp spanning the 5' region of $p d x A$ was PCR amplified from the genome of PAO1 using the primers SP_pdxA300_EcoRI_(F) and SP_pdxA300_

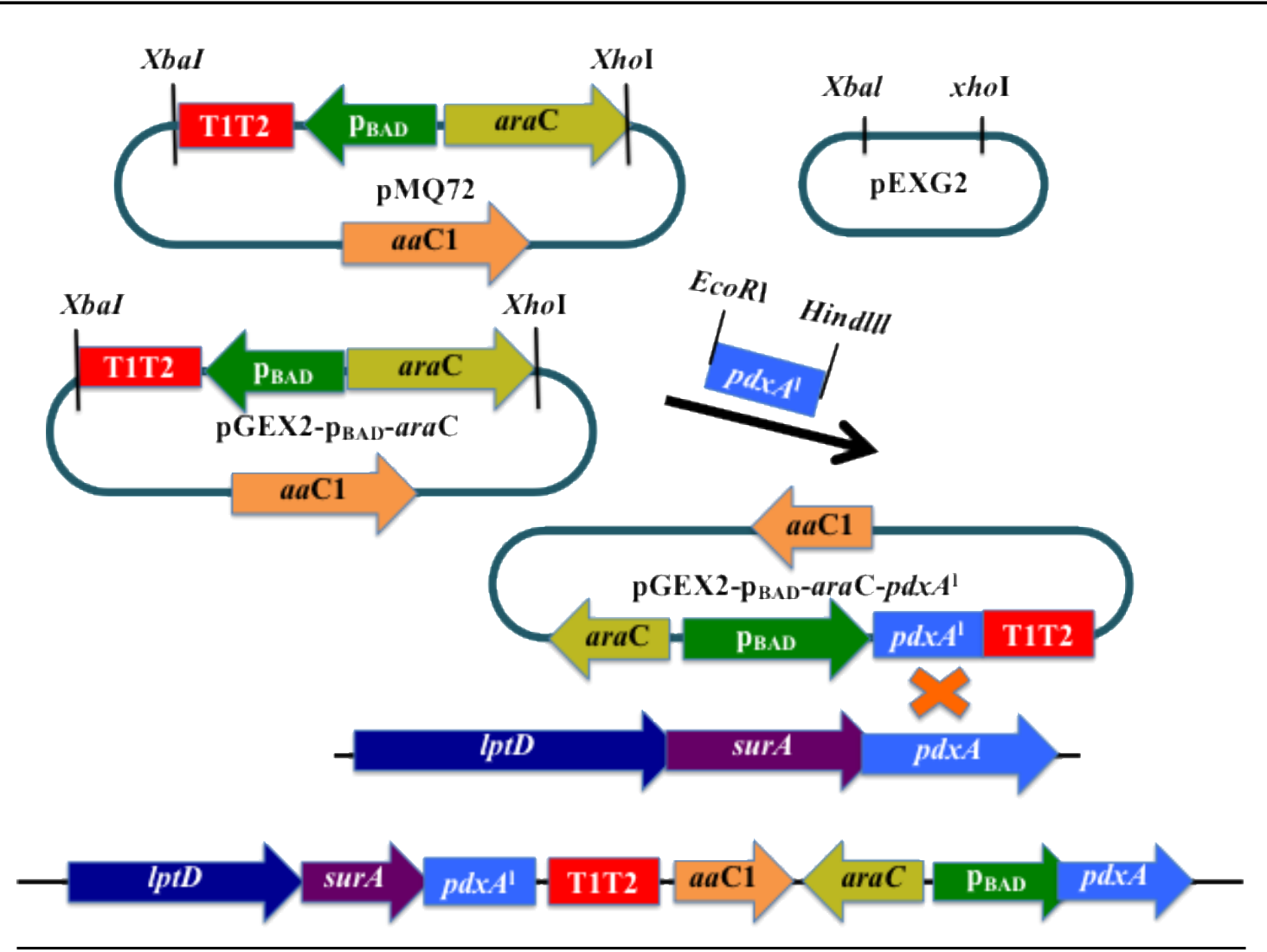

Figure 3.6. Strategy to construct $p d x A$ conditional mutant of $P$. aeruginosa. The methods of construction of $p d x A$ conditional mutation PAO1. 
HindIII_(R). The PCR product was digested with EcoRI and HindIII and ligated downstream of $\mathrm{P}_{\mathrm{BAD}}$ promoter into pMQ72 digested with EcoRI and HindIII and the plasmid was called as pMQ72-pdxA300. Resulting plasmid pMQ72-pdxA300 was used to amplify a 2,239-bp fragment containing $\operatorname{araC}-p d x A_{300-\mathrm{T} 1 \mathrm{~T} 2}$ using the primer pairs $\mathrm{pBAD}$ (F) and pBAD (R). The fragment was then digested with $\mathrm{XhoI}$ and $\mathrm{XbaI}$ and ligated into integration proficient vector $\mathrm{pEXG} 2$ digested by the same restriction enzymes resulting in the plasmid pEXG2P $\mathrm{PAD}_{\mathrm{BA}}: p d x A_{300}$. The plasmid pEXG2P $\mathrm{PAD}_{\mathrm{B}}: p d x A_{300}$ was used to transform E. coli. The plasmid was then transferred into P. aeruginosa PAO1 by triparental mating, with the selection of the conditional mutant on PIA (Difco) medium supplemented with arabinose $(0.5 \%)$ and $\mathrm{Gm}\left(100 \mu \mathrm{g} \mathrm{ml}^{-1}\right)$. The integration was confirmed by sequencing and PCR, resulting in the strain PAO1-pEXG2:pdxA300 (Figure 3.6).

\section{qPCR analysis of the first genes in the pathways, namely gapA, phzC, and $p h z E$}

We postulate that the presence of PLP will result in the decreased expression of gapA and increased expression $p h z C$ and $p h z E$ (Figure 3.3). Briefly, wild type and $p d x A$ mutant strain should be grown in $\mathrm{M} 9$ broth at $37^{\circ} \mathrm{C}$ in the presence of arabinose and glucose (to deplete PdxA) for 3 hours, and RNA should be isolated using RNeasy Mini Kit (Qiagen) as per the manufacturer's protocol. The RNA should be purified, and cDNA should be synthesized. The qPCR of the respective genes (Figure 3.2) should be performed to explore the possible pathway for pyocyanin production when $P$. aeruginosa is grown in the presence of PLP. 
CHAPTER 4

Discussion 
Living cells have a sophisticated regulatory mechanism that controls how they transduce the environmental clues downstream to the cell. One of the mechanisms that are evolutionarily conserved from humans to bacteria is termed regulated intramembrane proteolysis (RIP). The RIP process is predominantly involved in a progressive two-step proteolytic degradation initiated by the cleavage of a single-span transmembrane protein ultimately releasing extracellular and/or intracellular fragments (Brown et al., 2000; Lichtenthaler and Steiner, 2007; Qiu et al., 2007). The RIP system in cells functions in various ways, including proliferation, differentiation, protein degradation, cell adhesion, lipid metabolism, mitophagy, and transcriptional regulation (Damron and Yu, 2011; Qiu et al., 2007; Shanbhag et al., 2012; Yoshida et al., 2013). In this dissertation, we explored the activation of the RIP system of $P$. aeruginosa by an outer membrane protein referred as lipopolysaccharide transport protein $\mathrm{D}(\mathrm{LptD})$. The $l p t D$ is part of three gene operon $l p t D$ surA-pdxA. The PdxA is involved in synthesis of pyridoxal 5'-phosphate (PLP). Culturing P. aeruginosa in the LB supplemented with the of PLP shows the increase in biosynthesis of pyocyanin. The pyocyanin and aromatic amino acid are synthesized from chorismic acid. Culturing P. aeruginosa in LB media supplemented with aromatic amino acids; phenylalanine and tyrosine also increase the pyocyanin production.

A variety of organisms, including bacteria, fungi, parasites, viruses and other agents are the cause of nosocomial infections. Recently, using data from hospital-based surveillance studies and Infectious Diseases Society of America, a certain group of nosocomial pathogens has been referred to as ESKAPE pathogens (Peters et al., 2008; Pogue et al., 2015; Rice, 2010). The ESKAPE pathogens includes a group of both Gramnegative and Gram-positive bacteria made up of Enterococcus faecium, Staphylococcus 
aureus, Klebsiella pneumoniae, Acinetobacter baumannii, P. aeruginosa, and Enterobacter species. Among ESKAPE pathogens, $P$. aeruginosa is a formidable pathogen because of its metabolic versatility, intrinsic and acquired antibiotic resistance, biofilm formation, and production of multiple virulence factors. One of the virulence factors of $P$. aeruginosa is the exopolysaccharide alginate (Hoiby, 1975). Alginate production in the lung of the CF patients infected by $P$. aeruginosa indicates a chronic infection that leads to a poor prognosis (Hoiby et al., 1977; Pedersen et al., 1992). Thus, it is important to understand the genes involved and their mechanism of action in the production of alginate in $P$. aeruginosa. One part of this study focused on identifying novel genes associated with the production of alginate and pathways of alginate production while in the other section, we investigated the production of another virulence factor pyocyanin.

CF individuals are thought to be initially colonized by $P$. aeruginosa from diverse environmental sources as well as from CF clinics (Denton et al., 2002; Jensen et al., 1997; Romling et al., 1994; Scott and Pitt, 2004). Initial colonizing P. aeruginosa strains have non-mucoid phenotype with a wild-type mucA, however, mucoid mucA mutants eventually emerge and dominate the population of P. aeruginosa in the CF lung (Martin et al., 1993). In wild-type PAO1 MucA sequesters $\mathrm{AlgT} / \mathrm{U}$ at the inner membrane, preventing the sigma factor from directing RNA polymerase to transcribe the alginate transcriptome; however, when $m u c A$ is mutated, AlgT/U is free to guide RNA polymerase (Schurr et al., 1996). In the clinical setting, it has been found that $25 \%$ of mucoid $P$. aeruginosa isolated from $\mathrm{CF}$ individuals has a deletion of a single guanine nucleotide $(\mathrm{G})$ in a string of five $\mathrm{G}$ nucleotides located at 426-430 bp in mucA ORF (Martin et al., 1993; Schurr et al., 1996). In this study, we used a constitutively mucoid laboratory strain PDO300 (PAOmucA22) that was derived 
from non-mucoid PAO1 (Mathee et al., 1999). The mucoid PDO300 was grown under oxygen-limited conditions, a stressful situation that reverts the metabolically demanding mucoid PDO300 to non-mucoid phenotype which are referred to as the suppressor of alginate production (sap) mutants (Mathee et al., 1999). One of the sap mutants that is of interest in this dissertation is sap 27 . To identify mutations in sap 27 mutants, the strains were complemented with the PAO1 minimum tiling path (MTP) library that was previously divided into four pools (Sautter et al., 2012). The four pools of cosmids were introduced into the sap27 and mucoid phenotype of the strains was screened. The junctional sequencing revealed that cosmid pMO012217 restored the mucoidy of the sap27. Finally, by transposon mutagenizing the cosmid pMO012217 that restored the mucoid phenotype, we mapped lptD (PA0595) that can restore the mucoid phenotype of one of sap27 and lptDsurA-pdxA forms an operon (chapter 2). The $p d x A$ is involved in pyridoxal 5'-phosphate (PLP) synthesis in Gram-negative bacteria. Culturing P. aeruginosa in LB media supplemented with PLP as well as aromatic amino acids phenylalanine and tyrosine increases the production of pyocyanin (chapter 3). The pyocyanin is one of the virulent factors of P. aeruginosa (Gibson et al., 2009; Kanthakumar et al., 1993; Morales et al., 2010).

\section{Outer membrane protein LptD}

Previously, LptD was known as Imp and OstA (Abe et al., 2003; Aono et al., 1994; Sampson et al., 1989). The protein has been extensively studied and deemed as a promising vaccine antigen and potential immunotherapeutic target in several Gram-negative bacteria,

such as Neisseria gonorrhoeae, A. baumannii, Neisseria meningitidis, Vibrio 
parahaemolyticus and $P$. aeruginosa (Balibar and Grabowicz, 2016; Bojkovic et al., 2015; Bos et al., 2004; Zha et al., 2016). The role of LptD, along with other seven essential proteins (LptA-LptG), is to transport lipopolysaccharide (LPS) to the cell surface, thus involved in outer membrane biogenesis (Braun and Silhavy, 2002). LptD is an outer membrane protein (OMP) with a C-terminal $\beta$-barrel and an $\mathrm{N}$-terminal jellyroll domain containing three nonconsecutive disulfide bonds (Srinivas et al., 2010; Werneburg et al., 2012). Survival protein during stationary phase (SurA), 17-Kilodalton Protein (Skp), and FK506-binding protein A (FkpA) maintain the folding-competent state while LptD is translocating from the periplasm to the outer membrane (Bitto and McKay, 2002; Rouviere and Gross, 1996; Schwalm et al., 2013; Tormo et al., 1990). In the outer membrane, the Bam complex assembles LptD in coordination with LptE (Lee et al., 2016). The three disulfide bonds are rearranged to become a mature LptD-LptE complex (Moehle et al., 2016).

LPS is the major endotoxin that stimulates the host inflammatory response (Janda, 1972; Raetz and Whitfield, 2002; Rosenfeld et al., 2006). In mice, LptD induces a strong host immune response (Zha et al., 2016). LptD is an essential outer membrane protein of most of the Gram-negative bacteria and has the capacity to generate an immune response in humans, making the protein an ideal universal vaccine candidate (Srinivas et al., 2010; Zha et al., 2016). Photoaffinity labeling, liquid chromatography-electrospray ionization tandem mass spectrometry (LC-ESI-MS-MS) and a polyclonal antibody raised against a C-terminus domain of $\mathrm{LptD}$ show that the $\mathrm{LptD}$ is an outer membrane protein with a mass of approximately $100 \mathrm{kDa}$ (Srinivas et al., 2010). We also confirmed the finding that LptD localizes to the membrane fraction of P. aeruginosa (Figure 2.5). 


\section{The role of the second gene of the lptD-operon, surA, in LptD translocation}

In all organisms, proteins are translated primarily by the cytosolic ribosome and such proteins have to be transported to various cellular locations. In bacterial cells, three known translocation processes that transport the proteins across the cell membrane have been reported: (a) Sec-mediated translocation across the cytoplasmic membrane (Manting and Driessen, 2000); (b) twin-arginine transport-mediated translocation across the cytoplasmic membrane (Palmer and Berks, 2012); and (c) more specialized mechanisms for delivery of specific proteins to the outermost surface of the cell (Thanassi and Hultgren, 2000). Of these mechanisms, one of the major pathways of protein translocation from the cytosol across the inner membrane in bacteria is provided by the Sec machinery (Corey et al., 2016; Duong and Wickner, 1997; Nouwen and Driessen, 2002). Transportation of outer membrane proteins initiates with the translocation of nascent OMPs through the Sec machinery located in the inner membrane (Huber et al., 2017). Once in the periplasm, nascent OMPs interact with periplasmic chaperones such as SurA (Hennecke et al., 2005; Xu et al., 2007). SurA has been shown to interact directly with the A2 helix of $\beta$-barrel assembly machinery A (BamA) polypeptide transport-associated protein (POTRA 1) (Bennion et al., 2010). No data exists that shows the interactions with other periplasmic chaperones, including the 17- kilodalton protein (Skp) and degradation protein $\mathrm{P}(\mathrm{DegP})$. Chaperone-bound OMPs are then thought to be offloaded to the BamA POTRA domain by a mechanism known as $\beta$-augmentation, in which the $\beta$-strands of substrate OMPs align with the $\beta$-strand of POTRA in a sequence and orientation-independent manner (Workman et al., 2012). It has been shown in surA mutant of an E. coli that the transcription of lptD is not significantly affected but the LptD protein levels are decreased suggesting that in the 
periplasm SurA is the true chaperon of LptD (Vertommen et al., 2009). The LptD is a true substrate of SurA is further reinforced by the fact that in $\gamma$-proteobacteria, the $\operatorname{lptD-surA}$ operon is conserved even though nearby genes are not (Braun and Silhavy, 2002; Winsor et al., 2016). This further suggests that the LptD is a preferred substrate of SurA. Furthermore, it has been shown that though LptD is highly dependent on SurA as its preferential chaperone protein, other periplasmic chaperone proteins such as Skp and FkpA are also required for the assembly of LptD (Schwalm et al., 2013).

In E. coli, the domains of SurA consists of the N-terminal domain [aa 21 -162], P1 domains [aa 172-274], P2 domain [aa 282-385], and C-terminal domain [aa 388-428](Bitto and McKay, 2002). The N and C-terminals of SurA including P2 act as the core chaperone module whereas the P1 domain is known as an inactive peptidyl-prolyl-cis/trans isomerase (PPIase) domain. The P1 form makes noncovalent interactions with the core chaperone module that occludes the function of SurA. Both P1 and P2 domains are dispensable as mutants lacking these domains are sufficient for the chaperone function of SurA in vitro. SurA has been shown to bind to peptides that are rich in aromatic amino acids (Hennecke et al., 2005).

\section{Role of LptE in the proper folding of LptD in the outer membrane}

In E. coli, His-tagged LptD was used initially to show that LptE and LptD interact and later it was found that the proteins interact in 1:1 ratio (Chng et al., 2010; Wu et al., 2006). Through pull-down experiments, it was shown that the both the full length LptD as well as only C-termini LptD tagged with histidine (C-LptDHis) could pull down similar amounts of LptE, suggesting that the LptE interacts with the C-terminus of LptD (Chng et 
al., 2010). In E. coli, LptE helps in the proper rearrangement of disulfide bonds present in LptD but the exact mechanism is not known (Ruiz et al., 2010). LptD of P. aeruginosa is composed of 924 amino acids. The periplasmic domain of the $\mathrm{LptD}$ (34- 323) is 100 amino acids longer than LptD of $E$. coli and contains a pair of cysteine residues that could form a disulfide bond. In SDS-PAGE, heat denatured LptD with and without reduction with dithiothreitol (DTT) showed denatured and non-reduced LptD with an apparent size of 130 $\mathrm{kDa}$ and the DTT-reduced form with an apparent size of $100 \mathrm{kDa}$ suggesting the presence of a putative interdomain di-sulfide bond (Andolina et al., 2018). Additionally, by sequential replacement of all six cysteine residues to serine and examining the gel mobility of such modified LptD in SDS-PAGE showed that the protein is of $100 \mathrm{kDa}$, suggesting the presence of internal disulfide bonds of LptD (Andolina et al., 2018). The Ni-affinity pull down showed LptD was co-purified along with $\mathrm{LptE}_{\mathrm{His}}$, indicating both proteins interact with each other but how LptE helps in the rearrangement of disulfide bond present in LptD remains to be explored (Andolina et al., 2018).

\section{Alginate production}

In the lungs of patients with cystic fibrosis, $P$. aeruginosa starts with a non-mucoid phenotype and converts to an alginate overproducing mucoid phenotype, indicating of a chronic infection (Evans and Linker, 1973; Govan and Fyfe, 1978; Pressler et al., 2011a). It has been shown that motile $P$. aeruginosa eventually deposited into the hypoxic mucus zone in the lung of CF airway responds to such oxygen-limiting conditions by increasing alginate production (Worlitzsch et al., 2002). Alginate is a linear polymer of $\beta$-Dmannuronic acid and the $\alpha$-L-guluronic acid that has an ability to stimulate the production 
of antibodies IgA and IgG (Aanaes et al., 2013; Evans and Linker, 1973; Pedersen et al., 1990).

Alginate production in $P$. aeruginosa involves a very complex regulatory process where the key player is an alternative sigma factor AlgT/U (Devries and Ohman, 1994a; Martin et al., 1993a). The activity of AlgT/U is post-translationally inhibited by the antisigma factors MucA and MucB (Goldberg et al., 1993; Martin et al., 1993b; Martin et al., 1993; Mathee et al., 1997). The release of AlgT/U from MucA is regulated by a mechanism known as the regulated intramembrane proteolytic pathway (RIP)(Qiu et al., 2007). In the RIP pathway, MucA undergoes sequential degradation and such degradation process is stimulated by the action of the periplasmic/outer membrane protein MucE due to the accumulation of unfolded proteins in the periplasm or the stress in the outer membrane activates MucE (Qiu et al., 2007). The activated-MucE interacts with AlgO to activate the inner membrane proteolytic protein AlgW to cleave MucA (Cezairliyan and Sauer, 2009; Qiu et al., 2007). The AlgW-cleaved MucA undergoes further cleavage by MucP, eventually releasing AlgT/U with a small portion of MucA still bound to it (Damron and Yu, 2011; Qiu et al., 2007). A cytoplasmic protein, SspA, guides the complex of cleaved MucA-AlgT/U to ClpXP where the remaining portion of MucA is degraded and eventually AlgT/U is free to bind to RNA polymerase (Qiu et al., 2008b; Yin et al., 2013). The AlgT/U bound RNA polymerase directs the transcription of its own algT/U-operon and other transcription factors $\mathrm{AlgR}, \mathrm{AlgB}$, and AmrZ (Devries and Ohman, 1994a; Martin et al., 1994; Wozniak and Ohman, 1994; Wozniak et al., 2003). These transcription factors AlgR, $\mathrm{AlgB}$, and AmrZ along with RNA polymerase-AlgT/U complex direct the transcription of $\operatorname{algD}$ promoter for the biosynthesis of alginate (Baynham and Wozniak, 1996; Kato and 
Chakrabarty, 1991; Leech et al., 2008; Mohr et al., 1991; Mohr et al., 1992). AlgR not only regulates the $\operatorname{alg} D$-operon but also activates the transcription of $\operatorname{alg} C$ that encodes a phosphomannomutase or phosphoglucomutase, which is essential for alginate, Psl, and rhamnolipid production (Lizewski et al., 2004; Wozniak and Ohman, 1994; Zielinski et al., 1991). Another important regulator of alginate production in P. aeruginosa is diguanylate cyclase/phosphatase, which by regulating the local pool of bis- (3'-5')-cyclic dimeric GMP (c-di-GMP) in the vicinity of the pili Z (PilZ) domain of the inner membrane protein Alg44, positively influencing the alginate production in $P$. aeruginosa (Hay et al., 2009; Merighi et al., 2007; Remminghorst and Rehm, 2006).

\section{Role of LptD the alginate production: a potential model}

Our results show that an outer membrane protein, $\mathrm{LptD}$, acts as a positive regulator of alginate production (Chapter 2)(Pandey et al., 2018). We showed that in a mucA mutant background, LptD is capable of restoring the mucoid phenotype. To understand the hierarchy of LptD in the RIP cascade, algW was deleted in both PAO1 and PDO300. LptD rescued the mucoid phenotype in PDO $\Delta$ alg $W$ suggesting that in a mисA mutant background, LptD acts upstream of $\mathrm{AlgW}$ in the RIP cascade whereas, in the case of PDO $\triangle a \lg O, \mathrm{LptD}$ failed to restore the mucoid phenotype. But, increasing the production of LptD in sap27 restores the mucoid phenotype suggesting LptD might interact with AlgO to produce the mucoid phenotype in a mucA mutant background. However, in wild type PAO1, increased expression of $\mathrm{LptD}, \mathrm{AlgO}$ is dispensable for rescuing mucoid phenotype. Based on this evidence, we concluded that LptD dependent alginate production is strain specific (Figure 2.8). 


\section{Role of PLP in pyocyanin production in $P$. aeruginosa}

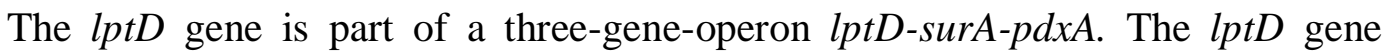
encodes an outer membrane protein (OMP) involved in lipopolysaccharide transport. Additionally, it has been shown that $l p t D$ positively influences alginate production (Pandey et al., 2018). SurA is the cognate chaperone protein of $\mathrm{LptD}$, and $\mathrm{PdxA}$ plays an important role in vitamin $\mathrm{B}_{6}$ synthesis (Figure 3.2) (Denoncin et al., 2010; Fitzpatrick et al., 2007; Laber et al., 1999; Rouviere and Gross, 1996). One of the aims of this dissertation was also to explore the role of vitamin $\mathrm{B}_{6}$ in the virulence of $P$. aeruginosa.

In E. coli, PdxA acts as an enzyme for the synthesis of vitamin $\mathrm{B}_{6}$ (Laber et al., 1999). The importance of vitamin B6 for bacterial pathogenesis has been demonstrated in Mycobacterium tuberculosis, Bacillus subtilis, Campylobacter jejuni, and Helicobacter pylori (Asakura et al., 2013; Dick et al., 2010; Grubman et al., 2010; Raschle et al., 2005). Our data suggest that vitamin $\mathrm{B}_{6}$, and the aromatic amino acids tyrosine and phenylalanine play a role in increased pyocyanin production in P. aeruginosa (Figure 3.3). But, the roles of PLP, tyrosine, and phenylalanine in pyocyanin production in $P$. aeruginosa remain unknown.

\section{Future directions}

This is the first report of an OMP component that plays a role in regulating alginate production. This study also opened many unanswered questions. Delineating the role of the LptD in the RIP cascade can help in identifying stress signals that trigger upstream or downstream proteolysis of MucA that releases the AlgT/U for mucoid conversion of $P$.

aeruginosa. By targeting LptD as a drug target, a tool can be developed to prevent alginate 
production. Secondly, MucD, $\mathrm{AlgO}, \mathrm{AlgW}$, and MucP are protease proteins. To achieve better treatment outcomes in chronic $P$. aeruginosa infections identifying the inhibitors of the proteases that can prevent alginate production can be a significant strategy. Finally, it is well established that microbes and plants can only synthesize pyridoxal 5'-phosphate (PLP) and humans have to depend on them for metabolic needs. By targeting the $P$. aeruginosa PLP biosynthesis pathway, growth or virulence factors of pathogenic microbes can be specifically inhibited that could result in the better prognosis for CF patients and prevent other infections by the microbe.

\section{Identify the role of LptD/OstA in alginate regulatory cascade}

To determine the role and point at which LptD/OstA is involved in the alginate regulatory cascade, $m и с D, m и c E, \operatorname{alg} W, \operatorname{alg} O$ and $m и с P$ can be expressed in the lptD mutant strain. Next, lptD/ostA can be expressed in $m и с D, \operatorname{alg} W, \operatorname{alg} O$ and $т и с P$ deletion strains. Then, the presence and absence of a mucoid phenotype can be scored. Based on the results, the location and role of the LptD/OstA in the alginate pathway can be deduced.

\section{Identify proteins interacting with LptD/OstA}

LptD might interact with a periplasmic protein to exert its effect on alginate production. To date, known periplasmic proteins associated with alginate production are $\mathrm{MucD}$, MucE, $\mathrm{AlgO}$, and $\mathrm{MucB}$. To determine the mechanism of action of LptD for alginate synthesis, it is imperative to identify the protein/s that interact with LptD. A co-immunoprecipitation assay can be done with His-tagged LptD to find its interacting partners. 
Conversely, the periplasmic proteins (MucB, AlgO, MucD, and MucE) can be His-tagged for the pull-down assay. The interacting partners can be identified using antibodies against the known proteins. In E. coli, SurA acts as a chaperone for $\mathrm{LptD}$ and $\mathrm{LptE}$ binds in the lumen of $\mathrm{LptD}$ (Chimalakonda et al., 2011; Chng et al., 2010; Denoncin et al., 2010; Moehle et al., 2016). This suggests that LptD might interact with other nonalginate periplasmic proteins. To test $\mathrm{LptD}$-SurA or $\mathrm{LptD}-\mathrm{LptE}$ interaction in $P$. aeruginosa, His-tagged SurA or LptE can be used in coimmunoprecipitation assays.

\section{LptD as a drug target}

$\mathrm{LptD}$ and the lipoprotein LptE are essential for the transport of lipopolysaccharide molecules from the periplasm to the outer leaflet of the outer membrane for the outer membrane biogenesis of in Gram-negative bacteria (Chng et al., 2010). It has been shown that macrocyclic peptidomimetics and the related molecule Murepavadin specifically target LptD inhibiting the LPS translocation in Pseudomonas spp. (Andolina et al., 2018; Srinivas et al., 2010; Werneburg et al., 2012). Our result showed $\mathrm{LptD}$ in $P$. aeruginosa is also involved in alginate production (Chapter 2 ). Thus, it is possible that the peptidomimetic drugs could also inhibit alginate production by $P$. aeruginosa, which has not been explored yet.

\section{Role of truncated AlgO in sap27}

LptD restored the mucoid phenotype of sap27 (Figure 2.3b) but could not restore the phenotype of PDO $\Delta$ algO. Both of the strains have 
mисA22 allele but truncated $\mathrm{AlgO}$ is present in the sap27 whereas, in the $\mathrm{PDO} \triangle a \lg O$, the gene is deleted. This led to the hypothesis that the truncated $\mathrm{AlgO}$ could still retain the function in sap27. It would be interesting to test whether the truncated $\mathrm{AlgO}$ has retained all or part of its function.

\section{Identification of small molecule inhibitors of alginate production}

The chronic infection in CF patients is the appearance of an increase in alginate production causing a mucoid phenotype in $P$. aeruginosa. The mucoid phenotype of the bacterium makes antibiotic treatments ineffective. The trypsin-like serine proteases ( $\mathrm{MucD}$ and $\mathrm{AlgW}$ ), a serine protease $(\mathrm{AlgO})$ and $\mathrm{Zn}$-dependent protease $(\mathrm{MucP})$ play a central role in the alginate production pathway in $P$. aeruginosa. Identification of specific proteinase inhibitors of MucD, AlgW, AlgO, and MucP could be useful therapeutic agents to prevent alginate production. Commercial proteinase inhibitors from G-biosciences or Sigma that can act on either of MucD, $\mathrm{AlgW}$, AlgO, and MucP. The available literature showed that between two inner membrane proteases $\mathrm{AlgW}$ and $\mathrm{MucP}$, the $\mathrm{MucP}$ is the essential protease for the proteolytic cleavage of both MucA or its mutant allele MucA22. MucP is $\mathrm{Zn}$ metalloproteinase. Thus, it may be worthwhile to use screening of $\mathrm{Zn}$ metalloproteinase inhibitors to impede alginate production by $P$. aeruginosa. Fortunately, a database for wide range of metalloproteinase inhibitors are available (Muvva et al., 2016). Culturing mucoid $P$. aeruginosa in the presence of the protease inhibitors and screening for the 
loss of mucoidy could be a strategy to identify potential inhibitors of specific protease proteins. Finally, the mechanism of action of the protease inhibitors can be explored.

\section{Identification of small molecule inhibitors of pyocyanin production}

Culturing $P$. aeruginosa in a media supplemented with PLP increased pyocyanin production. Pyocyanin is one of the virulence factors of $P$. aeruginosa. By identifying the proteins affecting pyocyanin production due to PLP, screening for small molecule inhibitors of the proteins can be done that could ultimately make $P$. aeruginosa less virulent. It is also important to note that PLP acts as a co-factor for 23 P. aeruginosa proteins. Thus, by inhibiting the activity of PLP synthesis, we can affect the metabolic activity of the microbe such that its growth and/or its virulence can be inhibited.

\section{Mechanism of cleavage MucA in the membrane}

Generally, the membrane-spanning segment of a protein forms $\alpha$ helices. Hydrogen bonds preclude the proteolytic cleavage of peptide bonds present in the $\alpha$ helices (Paetzel et al., 1998). How AlgW and MucP can get access to the peptide bond of MucA for the cleavage is still unresolved. It might be possible that due to the release of MucB, the MucA might undergo a conformational change or it could form a $\beta$ strand-like conformation that becomes more susceptible to proteolysis as previously predicted (Hubbard et al., 1994; Matthews, 1988). 


\section{Final conclusions and implications}

This dissertation identified an outer membrane protein referred to as Lipopolysaccharide transport protein $\mathrm{D}(\mathrm{LptD})$, as a component of alginate production pathway. The LptD is likely to interact with periplasmic protease protein $\mathrm{AlgO}$ and inner membrane protease protein $\mathrm{Alg} \mathrm{W}$ for restoration of the alginate production by suppressor of alginate production (sap) mutant sap27. The non-mucoid sap27 is derived from PDO300 that consecutively mucoid phenotype. The sap27 has a mutation in the AlgO.

The last gene of lptD-surA-pdxA forms an operon. It is well established that $p d x A$ is involved in pyridoxal 5'-phosphate synthesis (PLP), an active form of vitamin $\mathrm{B}_{6}$. Culturing the $P$. aeruginosa in excess PLP and aromatic amino acids phenylalanine and tyrosine increases pyocyanin production.

This investigation led to the finding of an outer membrane protein (OMP) that is important in alginate gene regulation in $P$. aeruginosa and this discovery could increase the chance of a potential treatment option. OMPs are an easier target because the effect of an efflux pump that might reduce the efficacy of the drug is bypassed. Additionally, data in this dissertation show that PLP and aromatic amino acids; phenylalanine and tyrosine enhance the production of pyocyanin. Targeting the proteins involved in the biosynthesis of PLP and aromatic amino acid could be a potential way to limit the infection caused by P. aeruginosa. 


\section{REFERENCES}

1. Aanaes K, Johansen HK, Poulsen SS et al. Secretory IgA as a diagnostic tool for Pseudomonas aeruginosa respiratory colonization. Journal of Cystic Fibrosis 2013;12: 81-7.

2. Aarons SJ, Sutherland IW, Chakrabarty AM et al. A novel gene, algK, from the alginate biosynthetic cluster of Pseudomonas aeruginosa. Microbiology 1997;143: 641-52.

3. Abe S, Okutsu T, Nakajima H et al. n-Hexane sensitivity of Escherichia coli due to low expression of imp/ostA encoding an $87 \mathrm{kDa}$ minor protein associated with the outer membrane. Microbiology 2003;149: 1265-73.

4. Ades SE, Connolly LE, Alba BM et al. The Escherichia coli sigma(E)-dependent extracytoplasmic stress response is controlled by the regulated proteolysis of an anti-sigma factor. Genes \& Development 1999;13: 2449-61.

5. Aiello AE, Cimiotti J, Delta-Latta P et al. A comparison of the bacteria found on the hands of 'homemakers' and neonatal intensive care unit nurses. Journal of Hospital Infection 2003;54: 310-5.

6. Akiyama Y, Kanehara K, Ito K. RseP (YaeL), an Escherichia coli RIP protease, cleaves transmembrane sequences. The EMBO Journal 2004;23: 4434-42.

7. Alba BM, Leeds JA, Onufryk C et al. DegS and YaeL participate sequentially in the cleavage of RseA to activate the sigma(E)-dependent extracytoplasmic stress response. Genes \& Development 2002;16: 2156-68.

8. Andolina G, Bencze LC, Zerbe K et al. A Peptidomimetic antibiotic interacts with the periplasmic domain of LptD from Pseudomonas aeruginosa. Acs Chemical Biology 2018;13: 666-75.

9. Aono R, Negishi T, Aibe K et al. Mapping of organic-solvent tolerance gene OstA in Escherichia-coli K-12. Bioscience Biotechnology and Biochemistry 1994;58: 1231-5. 
10. Asakura H, Hashii N, Uema $\mathbf{M}$ et al. Campylobacter jejuni pdxA affects flagellum-mediated motility to alter host colonization. Plos One 2013;8.

11. Bagge N, Schuster M, Hentzer M et al. Pseudomonas aeruginosa biofilms exposed to imipenem exhibit changes in global gene expression and beta-lactamase and alginate production. Antimicrobial Agents and Chemotherapy 2004;48: 117587.

12. Balasubramanian D, Kumari H, Jaric $\mathbf{M}$ et al. Deep sequencing analyses expands the Pseudomonas aeruginosa AmpR regulon to include small RNAmediated regulation of iron acquisition, heat shock and oxidative stress response. Nucleic Acids Research 2014;42: 979-98.

13. Balibar CJ, Grabowicz M. Mutant alleles of $l p t D$ Increase the permeability of Pseudomonas aeruginosa and define determinants of intrinsic resistance to antibiotics. Antimicrobial Agents and Chemotherapy 2016;60: 845-54.

14. Banks J, Cane DE. Biosynthesis of vitamin B6: direct identification of the product of the PdxA-catalyzed oxidation of 4-hydroxy-1-threonine-4-phosphate using electrospray ionization mass spectrometry. Bioorganic and Medicinal Chemistry Letters 2004;14: 1633-6.

15. Baron SS, Rowe JJ. Antibiotic action of pyocyanin. Antimicrobial Agents and Chemotherapy 1981;20: 814-20.

16. Bayes HK, Ritchie N, Irvine S et al. A murine model of early Pseudomonas aeruginosa lung disease with transition to chronic infection. Science Report 2016;6: 35838 .

17. Baynham PJ, Wozniak DJ. Identification and characterization of AlgZ, an AlgTdependent DNA-binding protein required for Pseudomonas aeruginosa algD transcription. Molecular Microbiology 1996;22: 97-108.

18. Bennion D, Charlson ES, Coon $\mathbf{E}$ et al. Dissection of beta-barrel outer membrane protein assembly pathways through characterizing BamA POTRA 1 mutants of Escherichia coli. Molecular Microbiology 2010;77: 1153-71. 
19. Berry A, Devault JD, Chakrabarty AM. High osmolarity is a signal for enhanced algD transcription in mucoid and nonmucoid Pseudomonas aeruginosa strains. Journal of Bacteriology 1989;171: 2312-7.

20. Bharathi S, Raman GV, Mohan DM et al. An unusual presentation of Pseudomonas aeruginosa blebitis following combined surgery. Indian Journal of Ophthalmology 2014;62: 958-93.

21. Bitto E, McKay DB. Crystallographic structure of SurA, a molecular chaperone that facilitates folding of outer membrane porins. Structure 2002;10: 1489-98.

22. Bodey GP, Bolivar R, Fainstein $\mathbf{V}$ et al. Infections caused by Pseudomonas aeruginosa. Reviews of Infectious Diseases 1983;5: 279-313.

23. Bojkovic J, Richie DL, Six DA et al. Characterization of an Acinetobacter baumannii lptD deletion strain: permeability defects and response to inhibition of lipopolysaccharide and fatty acid biosynthesis. Journal of Bacteriology 2015;198: $731-41$.

24. Bombieri C, Seia M, Castellani C. Genotypes and phenotypes in cystic fibrosis and cystic fibrosis transmembrane regulator-related disorders. Seminars in Respiratory and Critical Care Medicine 2015;36: 180-93.

25. Bordes $\mathbf{P}$, Lavatine $\mathbf{L}$, Phok $\mathbf{K}$ et al. Insights into the extracytoplasmic stress response of Xanthomonas campestris pv. campestris: role and regulation of \{sigma\}E-dependent activity. Journal of Bacteriology 2011;193: 246-64.

26. Bos MP, Tefsen B, Geurtsen $\mathbf{J}$ et al. Identification of an outer membrane protein required for the transport of lipopolysaccharide to the bacterial cell surface. Proceedings of the National Academy of Sciences of the United States of America 2004;101: 9417-22.

27. Bouchart F, Dubar A, Bessou JP et al. Pseudomonas aeruginosa coronary stent infection. Annals of Thoracic Surgery 1997;64: 1810-3.

28. Boucher JC, Martinez-Salazar J, Schurr MJ et al. Two distinct loci affecting conversion to mucoidy in Pseudomonas aeruginosa in cystic fibrosis encode homologs of the serine protease HtrA. Journal of Bacteriology 1996;178: 511-23. 
29. Boucher JC, Schurr MJ, Yu H et al. Pseudomonas aeruginosa in cystic fibrosis: role of тисC in the regulation of alginate production and stress sensitivity. Microbiology 1997a;143 3473-80.

30. Boucher JC, Yu H, Mudd MH et al. Mucoid Pseudomonas aeruginosa in cystic fibrosis: characterization of mис mutations in clinical isolates and analysis of clearance in a mouse model of respiratory infection. Infection and Immunity 1997b;65: 3838-46.

31. Boucher RC. Airway surface dehydration in cystic fibrosis: pathogenesis and therapy. Annual Review Medicine 2007;58: 157-70.

32. Boutin S, Weitnauer M, Hassel S et al. Real time quantitative PCR detection of Pseudomonas aeruginosa to discriminate intermittent from chronic infection in cystic fibrosis. Journal of Cystic Fibrosis 2018;17: 348-55.

33. Bragonzi A, Wiehlmann L, Klockgether $\mathbf{J}$ et al. Sequence diversity of the mисABD locus in Pseudomonas aeruginosa isolates from patients with cystic fibrosis. Microbiology 2006;152: 3261-9.

34. Braun M, Silhavy TJ. Imp/OstA is required for cell envelope biogenesis in Escherichia coli. Molecular Microbiology 2002;45: 1289-302.

35. Brencic A, McFarland KA, McManus HR et al. Th GacS/GacA signal transduction system of Pseudomonas aeruginosa acts exclusively through its control over the transcription of the RsmY and RsmZ regulatory small RNAs. Molecular Microbiology 2009;73: 434-45.

36. Brown MS, Ye J, Rawson RB et al. Regulated intramembrane proteolysis: a control mechanism conserved from bacteria to humans. Cell 2000;100: 391-8.

37. Carlson DM, Matthews LW. Polyuronic acids produced by Pseudomonas aeruginosa. Biochemistry 1966;5: 2817-22.

38. CDC. National action plan for combating antibiotic resistant bacteria; Pg: 25 National institute of health, 2015. 
39. Cezairliyan BO, Sauer RT. Inhibition of regulated proteolysis by RseB. Proceedings of the National Academy of Sciences of the United States of America 2007;104: 3771-6.

40. Cezairliyan BO, Sauer RT. Control of Pseudomonas aeruginosa AlgW protease cleavage of MucA by peptide signals and MucB. Molecular Microbiology 2009;72: 368-79.

41. CFF. Cystic fibrosis mutation database, 2011.

42. CFF. Cystic fibrosis foundation, 2016.

43. Chan BK, Turner PE, Kim S et al. Phage treatment of an aortic graft infected with Pseudomonas aeruginosa. Evolution Medicine and Public Health 2018;2018: 60-6.

44. Charlson ES, Werner JN, Misra R. Differential effects of $y f g L$ mutation on Escherichia coli outer membrane proteins and lipopolysaccharide. Journal of Bacteriology 2006;188: 7186-94.

45. Chen JH, Stoltz DA, Karp PH et al. Loss of anion transport without increased sodium absorption characterizes newborn porcine cystic fibrosis airway epithelia. Cell 2010;143: 911-23.

46. Chimalakonda G, Ruiz N, Chng SS et al. Lipoprotein LptE is required for the assembly of LptD by the beta-barrel assembly machine in the outer membrane of Escherichia coli. Proceedings of the National Academy of Sciences of the United States of America 2011;108: 2492-7.

47. Chitnis CE, Ohman DE. Cloning of Pseudomonas aeruginosa AlgG, which controls alginate structure. Journal of Bacteriology 1990;172: 2894-900.

48. Chitnis CE, Ohman DE. Genetic analysis of the alginate biosynthetic gene cluster of Pseudomonas aeruginosa shows evidence of an operonic structure. Molecular Microbiology 1993;8: 583-90.

49. Chng SS, Ruiz N, Chimalakonda G et al. Characterization of the two-protein complex in Escherichia coli responsible for lipopolysaccharide assembly at the 
outer membrane. Proceedings of the National Academy of Sciences of the United States of America 2010;107: 5363-8.

50. Choi KH, Kumar A, Schweizer HP. A 10-min method for preparation of highly electrocompetent Pseudomonas aeruginosa cells: Application for DNA fragment transfer between chromosomes and plasmid transformation. Journal of Microbiological Methods 2006;64: 391-7.

51. Chotirmall SH, Smith SG, Gunaratnam C et al. Effect of estrogen on Pseudomonas mucoidy and exacerbations in cystic fibrosis. New England Journal of Medicine 2012;366: 1978-86.

52. Choy MH, Stapleton F, Willcox MDP et al. Comparison of virulence factors in Pseudomonas aeruginosa strains isolated from contact lens- and non-contact lensrelated keratitis. Journal of Medical Microbiology 2008;57: 1539-46.

53. Chu L, May TB, Chakrabarty AM et al. Nucleotide sequence and expression of the AlgE gene involved in alginate biosynthesis by Pseudomonas aeruginosa. Gene 1991;107: 1-10.

54. Cigana C, Melotti P, Baldan R et al. Genotypic and phenotypic relatedness of Pseudomonas aeruginosa isolates among the major cystic fibrosis patient cohort in Italy. Bmc Microbiology 2016;16.

55. Ciofu O, Mandsberg LF, Bjarnsholt T et al. Genetic adaptation of Pseudomonas aeruginosa during chronic lung infection of patients with cystic fibrosis: strong and weak mutators with heterogeneous genetic backgrounds emerge in $m u c A$ and/or lasR mutants. Microbiology 2010;156: 1108-19.

56. Coburn B, Wang PW, Caballero JD et al. Lung microbiota across age and disease stage in cystic fibrosis. Scientific Reports 2015;5.

57. Cohen TS, Prince A. Cystic fibrosis: a mucosal immunodeficiency syndrome. Nature Medicine 2012;18: 509-19.

58. Cole SJ, Records AR, Orr MW et al. Catheter associated urinary tract infection by Pseudomonas aeruginosa is mediated by exopolysaccharide-independent biofilms. Infection and Immunity 2014;82: 2048-58. 
59. Collinet B, Yuzawa H, Chen T et al. RseB binding to the periplasmic domain of RseA modulates the RseA:sigmaE interaction in the cytoplasm and the availability of sigmaE.RNA polymerase. The Journal of Biological Chemistry 2000;275: 33898-904.

60. Colvin KM, Irie Y, Tart CS et al. The Pel and Psl polysaccharides provide Pseudomonas aeruginosa structural redundancy within the biofilm matrix. Environmental Microbiology 2012;14: 1913-28.

61. Corey RA, Allen WJ, Komar J et al. Unlocking the bacterial SecY translocon. Structure 2016;24: 518-27.

62. Costa KC, Bergkessel M, Saunders S et al. Enzymatic degradation of phenazines can generate energy and protect sensitive organisms from toxicity. MBio 2015;6.

63. Coste A, Gilain L, Roger G et al. Endoscopic and CT-scan evaluation of rhinosinusitis in cystic fibrosis. Rhinology 1995;33: 152-6.

64. Cui QN, Lv HN, Qi ZZ et al. Cross-regulation between the $p h z 1$ and $p h z 2$ operons maintain a balanced level of phenazine biosynthesis in Pseudomonas aeruginosa PAO1. Plos One 2016;11.

65. Cullen L, Weiser $\mathbf{R}$, Olszak $\mathbf{T}$ et al. Phenotypic characterization of an international Pseudomonas aeruginosa reference panel: strains of cystic fibrosis (CF) origin show less in vivo virulence than non-CF strains. Microbiology 2015;161: 1961-77.

66. Dalbey RE, Wang P, van Dijl JM. Membrane proteases in the bacterial protein secretion and quality control pathway. Microbiology and Molecular Biology Reviews 2012;76: 311-30.

67. Damron FH, Davis MR, Jr., Withers TR et al. Vanadate and triclosan synergistically induce alginate production by Pseudomonas aeruginosa strain PAO1. Molecular Microbiology 2011;81: 554-70.

68. Damron FH, Qiu D, Yu HWD. The Pseudomonas aeruginosa sensor kinase KinB negatively controls alginate production through $\mathrm{AlgW}$ dependent MucA proteolysis. Journal of Bacteriology 2009;191: 2285-95. 
69. Damron FH, Yu HD. Pseudomonas aeruginosa MucD regulates the alginate pathway through activation of MucA degradation via MucP proteolytic activity. Journal of Bacteriology 2011;193: 286-91.

70. Dapas JI, Rivero C, Burgos $\mathbf{P}$ et al. Pseudomonas aeruginosa infective endocarditis following aortic valve implantation: a note of caution. Open Cardiovascular Medicine Journal 2016;10: 28-34.

71. Darch SE, McNally A, Harrison F et al. Recombination is a key driver of genomic and phenotypic diversity in a Pseudomonas aeruginosa population during cystic fibrosis infection. Scientific Reports 2015;5.

72. Dartigalongue C, Missiakas D, Raina S. Characterization of the Escherichia coli sigma E regulon. The Journal of Biological Chemistry 2001;276: 20866-75.

73. Darzins A, Frantz B, Vanags RI et al. Nucleotide-sequence analysis of the Phosphomannose Isomerase Gene (Pmi) of Pseudomonas aeruginosa and comparison with the corresponding Escherichia coli gene manA. Gene 1986;42: 293-302.

74. Das T, Kutty SK, Tavallaie $\mathbf{R}$ et al. Phenazine virulence factor binding to extracellular DNA is important for Pseudomonas aeruginosa biofilm formation. Scientific Reports 2015;5.

75. Das T, Manefield M. Pyocyanin promotes extracellular DNA release in Pseudomonas aeruginosa. Plos One 2012;7.

76. Davidson IW, Sutherland IW, Lawson CJ. Localization of O-acetyl groups of bacterial alginate. Journal of General Microbiology 1977;98: 603-6.

77. De Boeck K, Amaral MD. Progress in therapies for cystic fibrosis. Lancet Respiratory Medicine 2016;4: 662-74.

78. De Las Penas A, Connolly L, Gross CA. The sigmaE-mediated response to extracytoplasmic stress in Escherichia coli is transduced by RseA and RseB, two negative regulators of sigmaE. Molecular Microbiology 1997;24: 373-85. 
79. DelicAttree I, Toussaint B, Froger A et al. Isolation of an IHF-deficient mutant of a Pseudomonas aeruginosa mucoid isolate and evaluation of the role of IHF in algD gene expression. Microbiology 1996;142: 2785-93.

80. Denoncin K, Vertommen D, Paek E et al. The protein-disulfide isomerase DsbC cooperates with SurA and DsbA in the assembly of the essential beta-barrel protein LptD. Journal of Biological Chemistry 2010;285: 29425-33.

81. Denton M, Kerr K, Mooney $\mathbf{L}$ et al. Transmission of colistin-resistant Pseudomonas aeruginosa between patients attending a pediatric cystic fibrosis center. Pediatric Pulmonology 2002;34: 257-61.

82. Deretic V, Dikshit R, Konyecsni WM et al. The AlgR gene, which regulates mucoidy in Pseudomonas aeruginosa, belongs to a class of environmentally responsive genes. Journal of Bacteriology 1989;171: 1278-83.

83. Deretic V, Konyecsni WM. Control of mucoidy in Pseudomonas aeruginosa: transcriptional regulation of $\operatorname{alg} R$ and identification of the second regulatory gene, algQ. Journal of Bacteriology 1989;171: 3680-8.

84. Devault JD, Kimbara K, Chakrabarty AM. Pulmonary dehydration and infection in cystic fibrosis - evidence that ethanol activates alginate geneexpression and induction of mucoidy in Pseudomonas aeruginosa. Molecular Microbiology 1990;4: 737-45.

85. Devries CA, Ohman DE. Mucoid-to-nonmucoid conversion in alginate producing Pseudomonas aeruginosa often results from spontaneous mutations in algT, encoding a putative alternate sigma factor, and shows evidence for autoregulation. Journal of Bacteriology 1994a;176: 6677-87.

86. Devries CA, Ohman DE. Mucoid-to-nonmucoid conversion in alginate producing Pseudomonas aeruginosa often results from spontaneous mutations in AlgT, encoding a putative alternate sigma-factor, and shows evidence for autoregulation. Journal of Bacteriology 1994b;176: 6677-87.

87. Dick T, Manjunatha U, Kappes B et al. Vitamin B 6 biosynthesis is essential for survival and virulence of Mycobacterium tuberculosis. Molecular Microbiology 2010;78: 980-8. 
88. Doggett RG, Harrison GM, Carter RE. Mucoid Pseudomonas aeruginosa in patients with chronic illnesses. Lancet 1971;1: 236-7.

89. Dong H, Xiang $\mathbf{Q}, \mathbf{G u} \mathbf{Y}$ et al. Structural basis for outer membrane lipopolysaccharide insertion. Nature 2014;511: 52-6.

90. Dray X, Kanaan R, Bienvenu T et al. Malnutrition in adults with cystic fibrosis. European Journal of Clinical Nutrition 2005;59: 152-4.

91. Duong F, Wickner W. Distinct catalytic roles of the SecYE, SecG and SecDFyajC subunits of preprotein translocase holoenzyme. EMBO Journal 1997;16: 2756-68.

92. Edmiston CE, Krepel CJ, Marks RM et al. Microbiology of explanted suture segments from infected and noninfected surgical patients. Journal of Clinical Microbiology 2013;51: 417-21.

93. Erickson JW, Gross CA. Identification of the sigma E subunit of Escherichia coli RNA polymerase: a second alternate sigma factor involved in high-temperature gene expression. Genes \& Development 1989;3: 1462-71.

94. Essar DW, Eberly L, Hadero A et al. Identification and characterization of genes for a second anthranilate synthase in Pseudomonas aeruginosa: interchangeability of the two anthranilate synthases and evolutionary implications. Journal of Bacteriology 1990;172: 884-900.

95. Evans LR, Linker A. Production and characterization of the slime polysaccharide of Pseudomonas aeruginosa. Journal of Bacteriology 1973;116: 915-24.

96. Farrell PM. The prevalence of cystic fibrosis in the European Union. Journal of Cystic Fibrosis 2008a;7: 450-3.

97. Farrell PM. The prevalence of cystic fibrosis in the European Union. Journal of Cystic Fibrosis 2008b;7: 450-3.

98. Faucz FR, Souza DAS, Olandoski M et al. CFTR allelic heterogeneity in Brazil: historical and geographical perspectives and implications for screening and counseling for cystic fibrosis in this country. Journal of Human Genetics 2010;55: 71-6. 
99. Figurski DH, Helinski DR. Replication of an origin-containing derivative of plasmid RK2 dependent on a plasmid function provided in trans. Proceedings of the National Academy of Sciences of the United States of America 1979;76: 164852.

100. Finan TM, Kunkel B, De Vos GF et al. Second symbiotic megaplasmid in Rhizobium meliloti carrying exopolysaccharide and thiamine synthesis genes. Journal of Bacteriology 1986;167: 66-72.

101. Finch S, McDonnell MJ, Abo-Leyah H et al. A Comprehensive analysis of the impact of Pseudomonas aeruginosa colonization on prognosis in adult bronchiectasis. European Respiratory Journal 2015;12: 1602-11.

102. Fitzpatrick TB, Amrhein N, Kappes B et al. Two independent routes of de novo vitamin B $_{6}$ biosynthesis: not that different after all. Biochemical Journal 2007;407: $1-13$.

103. Flynn JM, Levchenko I, Sauer RT et al. Modulating substrate choice: the SspB adaptor delivers a regulator of the extracytoplasmic-stress response to the AAA+ protease ClpXP for degradation. Genes \& Development 2004;18: 2292-301.

104. Fothergill JL, Walshaw MJ, Winstanley C. Transmissible strains of Pseudomonas aeruginosa in cystic fibrosis lung infections. European Respiratory Journal 2012;40: 227-38.

105. Frank LH, Demoss RD. On the biosynthesis of pyocyanine. Journal of Bacteriology 1959;77: 776-82.

106. Franklin MJ, Chitnis CE, Gacesa $\mathbf{P}$ et al. Pseudomonas aeruginosa AlgG is a polymer level alginate C5-mannuronan epimerase. Journal of Bacteriology 1994;176: 1821-30.

107. Franklin MJ, Nivens DE, Weadge JT et al. Biosynthesis of the Pseudomonas aeruginosa extracellular polysaccharides, alginate, Pel, and Psl. Frontiers in Microbiology 2011;2.

108. Franklin MJ, Ohman DE. Identification of $\operatorname{alg} F$ in the alginate biosynthetic gene cluster of Pseudomonas aeruginosa which is required for alginate acetylation. Journal of Bacteriology 1993;175: 5057-65. 
109. Franklin MJ, Ohman DE. Identification of algI and algJ in the Pseudomonas aeruginosa alginate biosynthetic gene cluster which are required for alginate $\mathrm{O}$ acetylation. Journal of Bacteriology 1996;178: 2186-95.

110. Franklin MJ, Ohman DE. Mutant analysis and cellular localization of the AlgI, AlgJ, and AlgF proteins required for O-acetylation of alginate in Pseudomonas aeruginosa. Journal of Bacteriology 2002;184: 3000-7.

111. Fujii A, Seki M, Higashiguchi M et al. Community-acquired, hospital-acquired, and healthcare-associated pneumonia caused by Pseudomonas aeruginosa. Respiratory Medicine Case Reports 2014;12: 30-3.

112. Fyfe JA, Govan JR. Alginate synthesis in mucoid Pseudomonas aeruginosa: a chromosomal locus involved in control. Journal of General Microbiology 1980;119: 443-50.

113. Gessard C. Sur les colorations bleue et verte des linges à pansements. Comptesrendus hebdomadaire des séances de l'Académie des Sciences 1882.

114. Ghafoor A, Hay ID, Rehm BH. Role of exopolysaccharides in Pseudomonas aeruginosa biofilm formation and architecture. Applied and Environmental Microbiology 2011;77: 5238-46.

115. Gibson J, Sood A, Hogan DA. Pseudomonas aeruginosa-Candida albicans interactions: localization and fungal toxicity of a phenazine derivative. Applied and Environmental Microbiology 2009;75: 504-13.

116. Glik J, Labus W, Kitala D et al. A 2000 patient retrospective assessment of a new strategy for burn wound management in view of infection prevention and treatment. International Wound Journal 2017.

117. Goldberg JB, Dahnke T. Pseudomonas aeruginosa AlgB, which modulates the expression of alginate, is a member of the NtrC subclass of prokaryotic regulators. Molecular Microbiology 1992;6: 59-66.

118. Goldberg JB, Gorman WL, Flynn JL et al. A mutation in algN permits trans activation of alginate production by algT in Pseudomonas species. Journal of Bacteriology 1993;175: 1303-8. 
119. Goldberg JB, Ohman DE. Construction and characterization of Pseudomonas aeruginosa algB mutants: role of algB in high-level production of alginate. Journal of Bacteriology 1987;169: 1593-602.

120. Gottesman S, Zipser D. Deg phenotype of Escherichia coli lon mutants. Journal of Bacteriology 1978;133: 844-51.

121. Govan JR. Mucoid strains of Pseudomonas aeruginosa: the influence of culture medium on the stability of mucus production. Journal of Medical Microbiology 1975;8: 513-22.

122. Govan JR, Fyfe JA. Mucoid Pseudomonas aeruginosa and cystic fibrosis: resistance of the mucoid from to carbenicillin, flucloxacillin and tobramycin and the isolation of mucoid variants in vitro. The Journal of Antimicrobial Chemotherapy 1978;4: 233-40.

123. Govan JR, Sarasola P, Taylor DJ et al. Isolation of a mucoid alginate producing Pseudomonas aeruginosa strain from the equine guttural pouch. Journal of Clinical Microbiology 1992;30: 595-9.

124. Grabert E, Wingender J, Winkler UK. An Outer membrane protein characteristic of mucoid strains of Pseudomonas aeruginosa. Fems Microbiology Letters 1990;68: 83-8.

125. Grabowicz M, Yeh J, Silhavy TJ. Dominant negative lptE mutation that supports a role for LptE as a plug in the LptD barrel. Journal of Bacteriology 2013;195: 1327-34.

126. Grisaru-Soen G, Lerner-Geva L, Keller $\mathbf{N}$ et al. Pseudomonas aeruginosa bacteremia in children: analysis of trends in prevalence, antibiotic resistance and prognostic factors. Pediatric Infectious Disease Journal 2000;19: 959-63.

127. Grobe S, Wingender J, Truper HG. Characterization of mucoid Pseudomonas aeruginosa strains isolated from technical water systems. Journal of Applied Bacteriology 1995;79: 94-102.

128. Grossowicz N, Hayat P, Halpern YS. Pyocyanin biosynthesis by Pseudomonas aeruginosa. Journal of General Microbiology 1957;16: 576-83. 
129. Grubman A, Phillips A, Thibonnier M et al. Vitamin $\mathrm{B}_{6}$ is required for full motility and virulence in Helicobacter pylori. Mbio 2010;1.

130. Haardt M, Benharouga M, Lechardeur D et al. C-terminal truncations destabilize the cystic fibrosis transmembrane conductance regulator without impairing its biogenesis - A novel class of mutation. Journal of Biological Chemistry 1999;274: 21873-7.

131. Haase G, Skopnik H, Groten $\mathbf{T}$ et $\boldsymbol{a l}$. Long-term fungal cultures from sputum of patients with cystic fibrosis. Mycoses 1991;34: 373-6.

132. Hammerle MM, Aleksandrov AA, Riordan JR. Disease-associated mutations in the extracytoplasmic loops of cystic fibrosis transmembrane conductance regulator do not impede biosynthetic processing but impair chloride channel stability. Journal of Biological Chemistry 2001;276: 14848-54.

133. Hassett DJ. Anaerobic production of alginate by Pseudomonas aeruginosa: Alginate restricts diffusion of oxygen. Journal of Bacteriology 1996;178: 7322-5.

134. Hatchette TF, Gupta R, Marrie TJ. Pseudomonas aeruginosa communityacquired pneumonia in previously healthy adults: Case report and review of the literature. Clinical Infectious Diseases 2000;31: 1349-56.

135. Hay ID, Remminghorst U, Rehm BHA. MucR, a novel membrane-associated regulator of alginate biosynthesis in Pseudomonas aeruginosa. Applied and Environmental Microbiology 2009;75: 1110-20.

136. Hennecke G, Nolte J, Volkmer-Engert R et al. The periplasmic chaperone SurA exploits two features characteristic of integral outer membrane proteins for selective substrate recognition. Journal of Biological Chemistry 2005;280: 235408.

137. Hentzer M, Teitzel GM, Balzer GJ et al. Alginate overproduction affects Pseudomonas aeruginosa biofilm structure and function. Journal of Bacteriology 2001;183: 5395-401.

138. Hershberger CD, Ye RW, Parsek MR et al. The algT (algU) gene of Pseudomonas aeruginosa, a key regulator involved in alginate biosynthesis, 
encodes an alternative sigma factor (sigma E). Proceedings of the National Academy of Sciences of the United States of America 1995;92: 7941-5.

139. Highsmith WE, Jr., Burch LH, Zhou $\mathbf{Z}$ et al. Identification of a splice site mutation $(2789+5 \mathrm{G}>\mathrm{A})$ associated with small amounts of normal CFTR mRNA and mild cystic fibrosis. Human Mutation 1997;9: 332-8.

140. Hilliam Y, Moore MP, Lamont IL et al. Pseudomonas aeruginosa adaptation and diversification in the non-cystic fibrosis bronchiectasis lung. European Respiratory Journal 2017;49.

141. Hodges NA, Gordon CA. Protection of Pseudomonas aeruginosa against ciprofloxacin and beta-Lactams by homologous alginate. Antimicrobial Agents and Chemotherapy 1991;35: 2450-2.

142. Hoiby N. Prevalence of mucoid strains of Pseudomonas aeruginosa in bacteriological specimens from patients with cystic fibrosis and patients with other diseases. Acta Pathologica et Microbiologica Scandinavica Supplement 1975;83: $549-52$.

143. Hoiby N, Flensborg EW, Beck B et al. Pseudomonas aeruginosa Infection in cystic-fibrosis - diagnostic and prognostic significance of Pseudomonas aeruginosa precipitins determined by means of crossed immunoelectrophoretic. Scandinavian Journal of Respiratory Diseases 1977;58: 65-79.

144. Holloway BW. Genetic recombination in Pseudomonas aeruginosa. Journal of General Microbiology 1955;13: 572-81.

145. Horton RM, Cai ZL, Ho SN $\boldsymbol{e t}$ al. Gene-splicing by overlap extension - tailormade genes using the polymerase chain-reaction. Biotechniques 1990;8: 528-5.

146. Hota S, Hirji Z, Stockton K et al. Outbreak of multidrug-resistant Pseudomonas aeruginosa colonization and infection secondary to imperfect intensive care unit room design. Infection Control and Hospital Epidemiology 2009;30: 25-33.

147. Hsueh PR, Teng LJ, Yang PC et al. Persistence of a multidrug-resistant Pseudomonas aeruginosa clone in an intensive care burn unit. Journal of Clinical Microbiology 1998;36: 1347-51. 
148. Huang B, Whitchurch CB, Croft L et al. A minimal tiling path cosmid library for functional analysis of the Pseudomonas aeruginosa PAO1 genome. Microbial and Comparative Genomics 2000;5: 189-203.

149. Huang YC, Lin TY, Wang CH. Community-acquired Pseudomonas aeruginosa sepsis in previously healthy infants and children: analysis of forty-three episodes. Pediatric Infectious Disease Journal 2002;21: 1049-52.

150. Hubbard SJ, Eisenmenger F, Thornton JM. Modeling studies of the change in conformation required for cleavage of limited proteolytic sites. Protein Science 1994;3: 757-68.

151. Huber D, Jamshad M, Hanmer $\mathbf{R}$ et al. SecA cotranslationally interacts with nascent substrate Proteins In Vivo. Journal of Bacteriology 2017;199.

152. Hughes KT, Mathee K. The anti-sigma factors. The Annual Review of Microbiology 1998;52: 231-86.

153. Huhulescu S, Simon M, Lubnow M et al. Fatal Pseudomonas aeruginosa pneumonia in a previously healthy woman was most likely associated with a contaminated hot tub. Infection 2011;39: 265-9.

154. Imperi F, Ciccosanti F, Perdomo AB et al. Analysis of the periplasmic proteome of Pseudomonas aeruginosa, a metabolically versatile opportunistic pathogen. Proteomics 2009;9: 1901-15.

155. Inaba K, Suzuki M, Maegawa K $\boldsymbol{e}$ t al. A pair of circularly permutated PDZ domains control RseP, the S2P family intramembrane protease of Escherichia coli. The Journal of Biological Chemistry 2008;283: 35042-52.

156. Jadhav $\mathbf{S}$, Sahasrabudhe T, Kalley $\mathbf{V}$ et al. The microbial colonization profile of respiratory devices and the significance of the role of disinfection: a blinded study. Journal of Clinical Diagnostic Research for doctors 2013;7: 1021-6.

157. Jahn M, Vorpahl C, Hubschmann T et al. Copy number variability of expression plasmids determined by cell sorting and Droplet Digital PCR. Microbial Cell Factories 2016;15. 
158. Jain S, Franklin MJ, Ertesvag $\mathbf{H}$ et al. The dual roles of AlgG in C-5epimerization and secretion of alginate polymers in Pseudomonas aeruginosa. Molecular Microbiology 2003;47: 1123-33.

159. Jain S, Ohman DE. Deletion of algK in mucoid Pseudomonas aeruginosa blocks alginate polymer formation and results in uronic acid secretion. Journal of Bacteriology 1998;180: 634-41.

160. Janda J. Endotoxins in a lipopolysaccharide preparation and cell envelope of Escherichia coli. Biochemical Journal 1972;130: 41P-2P.

161. Jelsbak L, Johansen HK, Frost AL et al. Molecular epidemiology and dynamics of Pseudomonas aeruginosa populations in lungs of cystic fibrosis patients. Infection and Immunity 2007;75: 2214-24.

162. Jensen ET, Giwercman B, Ojeniyi B et al. Epidemiology of Pseudomonas aeruginosa in cystic fibrosis and the possible role of contamination by dental equipment. Journal of Hospital Infection 1997;36: 117-22.

163. Kanehara K, Akiyama Y, Ito K. Characterization of the yaeL gene product and its S2P-protease motifs in Escherichia coli. Gene 2001;281: 71-9.

164. Kanehara K, Ito K, Akiyama Y. YaeL proteolysis of RseA is controlled by the PDZ domain of YaeL and a Gln-rich region of RseA. The EMBO Journal 2003;22: 6389-98.

165. Kanthakumar K, Taylor G, Tsang KW et al. Mechanisms of action of Pseudomonas aeruginosa pyocyanin on human ciliary beat in vitro. Infection and Immunity 1993;61: 2848-53.

166. Kartner N, Hanrahan JW, Jensen TJ et al. Expression of the cystic fibrosis gene in non-epithelial invertebrate cells produces a regulated anion conductance. Cell 1991;64: 681-91.

167. Kato J, Chakrabarty AM. Purification of the regulatory protein AlgR1 and its binding in the far upstream region of the AlgD promoter in Pseudomonas aeruginosa. Proceedings of the National Academy of Sciences of the United States of America 1991;88: 1760-4. 
168. Kay WW, Gronlund AF. Transport of aromatic amino acids by Pseudomonas aeruginosa. Journal of Bacteriology 1971;105: 1039-46.

169. Kinch LN, Ginalski K, Grishin NV. Site-2 protease regulated intramembrane proteolysis: sequence homologs suggest an ancient signaling cascade. Protein Science 2006;15: 84-93.

170. Knowlton RG, Cohenhaguenauer O, Cong NV et al. A polymorphic DNA marker linked to Cystic Fibrosis is located on chromosome-7. Nature 1985;318: $380-2$.

171. Kulczycki LL, Murphy TM, Bellanti JA. Pseudomonas colonization in cystic fibrosis. A study of 160 patients. Journal of American Medical Association 1978;240: 30-4.

172. Laber B, Maurer W, Scharf S et al. Vitamin B6 biosynthesis: formation of pyridoxine 5'-phosphate from 4-(phosphohydroxy)-L-threonine and 1-deoxy-Dxylulose-5-phosphate by PdxA and PdxJ protein. FEBS Letter 1999;449: 45-8.

173. Lambiase A, Raia V, Del Pezzo M et al. Microbiology of airway disease in a cohort of patients with cystic fibrosis. BMC Infectious Diseases 2006;6: 4.

174. Lau GW, Ran HM, Kong FS et al. Pseudomonas aeruginosa pyocyanin is critical for lung infection in mice. Infection and Immunity 2004;72: 4275-8.

175. Lazar SW, Almiron M, Tormo A et al. Role of the Escherichia coli SurA protein in stationary-phase survival. Journal of Bacteriology 1998;180: 5704-11.

176. Lee DG, Kim SH, Kim SY et al. Evidence-based guidelines for empirical therapy of neutropenic fever in Korea. Korean Journal of Internal Medicine 2011;26: 22052.

177. Lee J, Xue M, Wzorek JS et al. Characterization of a stalled complex on the betabarrel assembly machine. Proceedings of the National Academy of Sciences of the United States of America 2016;113: 8717-22.

178. Lee J, Zhang LH. The hierarchy quorum sensing network in Pseudomonas aeruginosa. Protein \& Cell 2015;6: 26-41. 
179. Lee TW, Brownlee KG, Conway SP et al. Evaluation of a new definition for chronic Pseudomonas aeruginosa infection in cystic fibrosis patients. Journal of Cystic Fibrosis 2003;2: 29-34.

180. Leech AJ, Sprinkle A, Wood L et al. The NtrC family regulator AlgB, which controls alginate biosynthesis in mucoid Pseudomonas aeruginosa, binds directly to the algD promoter. Journal of Bacteriology 2008;190: 581-9.

181. Leid JG, Willson CJ, Shirtliff ME et al. The exopolysaccharide alginate protects Pseudomonas aeruginosa biofilm bacteria from IFN-gamma-mediated macrophage killing. Journal of Immunology 2005;175: 7512-8.

182. Lewenza S, Gardy JL, Brinkman FS et al. Genome-wide identification of Pseudomonas aeruginosa exported proteins using a consensus computational strategy combined with a laboratory-based PhoA fusion screen. Genome Research 2005; 15: 321-9.

183. Li JG, Hao CY, Ren LL et al. Data mining of lung microbiota in cystic fibrosis patients. Plos One 2016;11.

184. Lichtenthaler SF, Steiner H. Sheddases and intramembrane-cleaving proteases: RIPpers of the membrane - Symposium on regulated intramembrane proteolysis. EMBO Reports 2007;8: 537-41.

185. Lichtinger A, Yeung SN, Kim $\mathbf{P}$ et al. Shifting trends in bacterial keratitis in Toronto: an 11-year review. Ophthalmology 2012;119: 1785-90.

186. Lieberman D, Lieberman D. Pseudomonal infections in patients with COPD: epidemiology and management. American Journal of Respiratory Medicine 2003;2: 459-68.

187. Lima S, Guo MS, Chaba $\mathbf{R}$ et al. Dual molecular signals mediate the bacterial response to outer-membrane stress. Science 2013;340: 837-41.

188. Lipinska B, Fayet $\mathbf{O}$, Baird $\mathbf{L}$ et al. Identification, characterization, and mapping of the Escherichia coli htrA gene, whose product is essential for bacterial growth only at elevated temperatures. Journal of Bacteriology 1989;171: 1574-84. 
189. Lipinska B, Sharma S, Georgopoulos C. Sequence analysis and regulation of the htrA gene of Escherichia coli: a sigma 32-independent mechanism of heatinducible transcription. Nucleic Acids Research 1988;16: 10053-67.

190. Lipinska B, Zylicz M, Georgopoulos C. The HtrA (DegP) protein, essential for Escherichia coli survival at high temperatures, is an endopeptidase. Journal of Bacteriology 1990;172: 1791-7.

191. Lizewski SE, Schurr JR, Jackson DW et al. Identification of AlgR-regulated genes in Pseudomonas aeruginosa by use of microarray analysis. Journal of Bacteriology 2004;186: 5672-84.

192. Lonetto M, Gribskov M, Gross CA. The sigma 70 family: sequence conservation and evolutionary relationships. Journal of Bacteriology 1992;174: 3843-9.

193. Lonetto MA, Brown KL, Rudd KE et al. Analysis of the Streptomyces coelicolor sigE gene reveals the existence of a subfamily of eubacterial RNA polymerase sigma factors involved in the regulation of extracytoplasmic functions. Proceedings of the National Academy of Sciences of the United States of America 1994;91:75737.

194. Lucas N, Humble M, Sim D et al. Temporal changes in neutropenic blood culture isolates and disease associations: a single center series of 1139 episodes. Internal Medicine Journal 2017;47: 962-5.

195. Ma LY, Wang J, Wang SW et al. Synthesis of multiple Pseudomonas aeruginosa biofilm matrix exopolysaccharides is post-transcriptionally regulated. Environmental Microbiology 2012;14: 1995-2005.

196. Ma S, Selvaraj U, Ohman DE et al. Phosphorylation-independent activity of the response regulators $\mathrm{AlgB}$ and $\mathrm{AlgR}$ in promoting alginate biosynthesis in mucoid Pseudomonas aeruginosa. Journal of Bacteriology 1998;180: 956-68.

197. Ma S, Wozniak DJ, Ohman DE. Identification of the histidine protein kinase KinB in Pseudomonas aeruginosa and its phosphorylation of the alginate regulator AlgB. The Journal of Biological Chemistry 1997a;272: 17952-60. 
198. Ma S, Wozniak DJ, Ohman DE. Identification of the histidine protein kinase KinB in Pseudomonas aeruginosa and its phosphorylation of the alginate regulator AlgB. Journal of Biological Chemistry 1997b;272: 17952-60.

199. MacEachran DP, Ye S, Bomberger JM et al. The Pseudomonas aeruginosa secreted protein PA2934 decreases apical membrane expression of the cystic fibrosis transmembrane conductance regulator. Infection and Immunity 2007;75: $3902-12$.

200. Maharaj R, May TB, Wang SK et al. Sequence of the alg8 and alg44 genes involved in the synthesis of alginate by Pseudomonas-aeruginosa. Gene 1993;136: 267-9.

201. Maharaj S, Isache C, Seegobin K et al. Necrotizing Pseudomonas aeruginosa community-acquired pneumonia: A case report and review of the literature. Case Reports in Infectious Diseases 2017.

202. Mai GT, Seow WK, Pier GB et al. Suppression of Lymphocyte and Neutrophil functions by Pseudomonas aeruginosa mucoid exopolysaccharide (Alginate) reversal by physicochemical, alginase, and specific monoclonal-antibody treatments. Infection and Immunity 1993;61: 559-64.

203. Manting EH, Driessen AJM. Escherichia coli translocase: the unravelling of a molecular machine. Molecular Microbiology 2000;37: 226-38.

204. Marson FAL, Bertuzzo CS, Ribeiro JD. Classification of CFTR mutation classes. Lancet Respiratory Medicine 2016;4: E37-E8.

205. Martin DR. Mucoid variation in Pseudomonas aeruginosa induced by the action of phage. Journal of Medical Microbiology 1973;6: 111-8.

206. Martin DW, Holloway BW, Deretic V. Characterization of a locus determining the mucoid status of Pseudomonas aeruginosa: AlgU shows sequence similarities with a Bacillus sigma factor. Journal of Bacteriology 1993a;175: 1153-64.

207. Martin DW, Schurr MJ, Mudd MH et al. Differentiation of Pseudomonas aeruginosa into the alginate-producing form: inactivation of conversion to mucoidy. Molecular Microbiology 1993b;9: 497-506. 
208. Martin DW, Schurr MJ, Mudd MH et al. Mechanism of conversion to mucoidy in Pseudomonas aeruginosa infecting cystic fibrosis patients. Proceeding of the National Academy of Sciences of the United States of America 1993;90: 8377-81.

209. Martin DW, Schurr MJ, Yu H et al. Analysis of promoters controlled by the putative sigma-factor AlgU regulating conversion to mucoidy in Pseudomonas aeruginosa relationship to sigma(E) and stress response. Journal of Bacteriology 1994;176: 6688-96.

210. Martinez-Solano L, Macia MD, Fajardo A et al. Chronic Pseudomonas aeruginosa Infection in Chronic Obstructive Pulmonary Disease. Clinical Infectious Diseases 2008;47: 1526-33.

211. Mathee K, Ciofu O, Sternberg $\mathbf{C}$ et al. Mucoid conversion of Pseudomonas aeruginosa by hydrogen peroxide: a mechanism for virulence activation in the cystic fibrosis lung. Microbiology 1999;145 1349-57.

212. Mathee K, McPherson CJ, Ohman DE. Posttranslational control of the algT $(\operatorname{alg} U)$-encoded sigma22 for expression of the alginate regulon in Pseudomonas aeruginosa and localization of its antagonist proteins MucA and MucB (AlgN). Journal of Bacteriology 1997; 179: 3711-20.

213. Matthews BW. Structural basis of the action of thermolysin and related zinc peptidases. Accounts of Chemical Research 1988;21: 333-40.

214. Mauch RM, Norregaard LL, Ciofu O et al. IgG avidity to Pseudomonas aeruginosa over the course of chronic lung biofilm infection in cystic fibrosis. Journal of Cystic Fibrosis 2017, DOI 10.1016/j.jcf.2017.09.004.

215. Mavrodi DV, Bonsall RF, Delaney SM et al. Functional analysis of genes for biosynthesis of pyocyanin and phenazine-1-carboxamide from Pseudomonas aeruginosa PAO1. Journal of Bacteriology 2001;183: 6454-65.

216. May TB, Shinabarger D, Boyd A et al. Identification of amino-acid-residues involved in the activity of phosphomannose isomerase-guanosine 5'-diphospho-Dmannose pyrophosphorylase - a bifunctional enzyme in the alginate biosyntheticpathway of Pseudomonas aeruginosa. Journal of Biological Chemistry 1994;269: $4872-7$. 
217. Mazzola M, Cook RJ, Thomashow LS et al. Contribution of phenazine antibiotic biosynthesis to the ecological competence of fluorescent pseudomonads in soil habitats. Applied and Environmental Microbiology 1992;58: 2616-24.

218. McAvoy MJ, Newton V, Paull A et al. Isolation of mucoid strains of Pseudomonas aeruginosa from non-cystic-fibrosis patients and characterization of the structure of their secreted alginate. Journal of Medical Microbiology 1989;28: 183-9.

219. McNeil SA, Nordstrom-Lerner L, Malani PN et al. Outbreak of sternal surgical site infections due to Pseudomonas aeruginosa traced to a scrub nurse with onychomycosis. Clinical Infectious Diseases 2001;33: 317-23.

220. Meluleni GJ, Grout M, Evans DJ et al. Mucoid Pseudomonas aeruginosa growing in a biofilm in vitro are killed by opsonic antibodies to the mucoid exopolysaccharide capsule but not by antibodies produced during chronic lung infection in cystic fibrosis patients. Journal of Immunology 1995;155: 2029-38.

221. Merighi M, Lee VT, Hyodo M et al. The second messenger bis-(3 '-5 ')-cyclicGMP and its PilZ domain-containing receptor Alg44 are required for alginate biosynthesis in Pseudomonas aeruginosa. Molecular Microbiology 2007;65: 87695.

222. Min KB, Lee KM, Oh YT et al. Nonmucoid conversion of mucoid Pseudomonas aeruginosa induced by sulfate stimulated growth. Fems Microbiology Letters 2014;360: 157-66.

223. Missiakas D, Mayer MP, Lemaire M et al. Modulation of the Escherichia coli sigmaE (RpoE) heat-shock transcription-factor activity by the RseA, RseB and RseC proteins. Molecular Microbiology 1997;24: 355-71.

224. Moehle K, Kocherla H, Bacsa B et al. Solution Structure and Dynamics of LptE from Pseudomonas aeruginosa. Biochemistry 2016;55: 2936-43.

225. Mohr CD, Deretic V. In vitro interactions of the histone-like protein IHF with the $a l g D$ promoter, a critical site for control of mucoidy in Pseudomonas aeruginosa. Biochemistry Biophysics Research Communication 1992;189: 837-44. 
226. Mohr CD, Hibler NS, Deretic V. AlgR, a response regulator controlling mucoidy in Pseudomonas aeruginosa, binds to the Fus sites of the $\operatorname{alg} D$ promoter located unusually far upstream from the messenger-RNA start site. Journal of Bacteriology 1991;173: 5136-43.

227. Mohr CD, Leveau JHJ, Krieg DP et al. AlgR-binding sites within the algD promoter make up a set of inverted repeats separated by a large intervening segment of DNA. Journal of Bacteriology 1992;174: 6624-33.

228. Monday SR, Schiller NL. Alginate synthesis in Pseudomonas aeruginosa: the role of AlgL (alginate lyase) and AlgX. Journal of Bacteriology 1996;178: 625-32.

229. Moradali MF, Donati I, Sims IM et al. Alginate polymerization and modification are linked in Pseudomonas aeruginosa. MBio 2015;6.

230. Morales DK, Jacobs NJ, Rajamani S et al. Antifungal mechanisms by which a novel Pseudomonas aeruginosa phenazine toxin kills Candida albicans in biofilms. Molecular Microbiology 2010;78: 1379-92.

231. Mousa HA. Aerobic, anaerobic and fungal burn wound infections. Journal of Hospital Infection 1997;37: 317-23.

232. Muvva C, Patra S, Venkatesan S. MMpI: A wide range of available compounds of matrix metalloproteinase inhibitors. Plos One 2016;11.

233. Nikolskaya AN, Galperin MY. A novel type of conserved DNA-binding domain in the transcriptional regulators of the AlgR/AgrA/LytR family. Nucleic Acids Research 2002;30: 2453-9.

234. Nixon GM, Armstrong DS, Carzino $\mathbf{R}$ et al. Clinical outcome after early Pseudomonas aeruginosa infection in cystic fibrosis. Journal of Pediatrics 2001;138: 699-704.

235. Nouwen N, Driessen AJ. SecDFyajC forms a heterotetrameric complex with YidC. Molecular Microbiology 2002;44: 1397-405. 
236. Oglesby-Sherrouse AG, Djapgne L, Nguyen AT et al. The complex interplay of iron, biofilm formation, and mucoidy affecting antimicrobial resistance of Pseudomonas aeruginosa. Pathogens and Disease 2014;70: 307-20.

237. Ohman DE, Chakrabarty AM. Genetic mapping of chromosomal determinants for the production of the exopolysaccharide alginate in a Pseudomonas aeruginosa cystic fibrosis isolate. Infection and Immunity 1981;33: 142-8.

238. Ohman DE, K. Mathee, C. J. McPherson, C. A. DeVries, S. Ma, D. J. Wozniak, and M. J. Franklin. Regulation of the alginate $(\operatorname{algD})$ operon in Pseudomonas aeruginosa. In: T. Nakazawa KF, D. Haas, and S. Silver (ed.) Molecular Biology of Pseudomonads. Washington, D.C.: American Society for Microbiology, 1996, 472-83.

239. Olejnickova K, Hola V, Ruzicka F. Catheter-related infections caused by Pseudomonas aeruginosa: virulence factors involved and their relationships. Pathogens and Disease 2014;72: 87-94.

240. Oliver A, Canton R, Campo P et al. High frequency of hypermutable Pseudomonas aeruginosa in cystic fibrosis lung infection. Science 2000;288: 12514.

241. Oliver AM, Weir DM. The effect of Pseudomonas alginate on rat alveolar macrophage phagocytosis and bacterial opsonization. Clinical and Experimental Immunology 1985;59: 190-6.

242. Olvera C, Goldberg JB, Sanchez $\mathbf{R}$ et al. The Pseudomonas aeruginosa algC gene product participates in rhamnolipid biosynthesis. Fems Microbiology Letters 1999;179: 85-90.

243. Paetzel M, Dalbey RE, Strynadka NC. Crystal structure of a bacterial signal peptidase in complex with a beta-lactam inhibitor. Nature 1998;396: 186-90.

244. Pallen MJ, Wren BW. The HtrA family of serine proteases. Molecular Microbiology 1997;26: 209-21.

245. Palmer GC, Jorth PA, Whiteley M. The role of two Pseudomonas aeruginosa anthranilate synthases in tryptophan and quorum signal production. Microbiology 2013;159: 959-69. 
246. Palmer KL, Aye LA, Whiteley M. Nutritional cues control Pseudomonas aeruginosa multicellular Behavior in cystic fibrosis sputum. Journal of Bacteriology 2007;189: 8079-87.

247. Palmer T, Berks BC. The twin-arginine translocation (Tat) protein export pathway. Nature Reviews Microbiology 2012;10: 483-96.

248. Pandey S, Delgado C, Kumari H et al. Outer-membrane protein LptD (PA0595) plays a role in the regulation of alginate synthesis in Pseudomonas aeruginosa. Journal of Medical Microbiology 2018, DOI 10.1099/jmm.0.000752.

249. Pedersen SS, Espersen F, Hoiby $\mathbf{N}$ et al. Immunoglobulin A and immunoglobulin G antibody responses to alginates from Pseudomonas aeruginosa in patients with cystic fibrosis. Journal of Clinical Microbiology 1990;28: 747-55.

250. Pedersen SS, Hoiby N, Espersen F et al. Role of alginate in infection with mucoid Pseudomonas aeruginosa in cystic fibrosis. Thorax 1992;47: 6-13.

251. Percudani R, Peracchi A. A genomic overview of pyridoxal-phosphate-dependent enzymes. EMBO Reports 2003;4: 850-4.

252. Percudani R, Peracchi A. The B6 database: a tool for the description and classification of vitamin B6-dependent enzymatic activities and of the corresponding protein families. Bmc Bioinformatics 2009;10.

253. Peters NK, Dixon DM, Holland SM et al. The research agenda of the National Institute of Allergy and Infectious Diseases for antimicrobial resistance. Journal of Infectious Diseases 2008;197: 1087-93.

254. Pier GB. Role of the cystic fibrosis transmembrane conductance regulator in innate immunity to Pseudomonas aeruginosa infections. Proceedings of the National Academy of Sciences of the United States of America 2000;97: 8822-8.

255. Pier GB, Coleman F, Grout M et al. Role of alginate O-acetylation in resistance of mucoid Pseudomonas aeruginosa to opsonic phagocytosis. Infection and Immunity 2001;69: 1895-901. 
256. Pillarisetti N, Williamson E, Linnane B et al. Infection, inflammation, and lung function decline in infants with cystic fibrosis. American Journal of Respiratory and Critical Care Medicine 2011;184: 75-81.

257. Pogue JM, Kaye KS, Cohen DA et al. Appropriate antimicrobial therapy in the era of multidrug-resistant human pathogens. Clinical Microbiology and Infection 2015;21: 302-12.

258. Ponting CP. Evidence for PDZ domains in bacteria, yeast, and plants. Protein Science 1997;6: 464-8.

259. Prasad R, Sharma H, Kaur G. Molecular basis of cystic fibrosis disease: an Indian perspective. Indian Journal of Clinical Biochemistry 2010;25: 335-41.

260. Pressler T, Bohmova C, Conway S et al. Chronic Pseudomonas aeruginosa infection definition: EuroCareCF Working Group report. Journal of Cystic Fibrosis 2011a;10: S75-S8.

261. Pressler T, Bohmova C, Conway S et al. Chronic Pseudomonas aeruginosa infection definition: EuroCareCF Working Group report. Journal of Cystic Fibrosis 2011b;10 Suppl 2: S75-8.

262. Price-Whelan A, Dietrich LE, Newman DK. Pyocyanin alters redox homeostasis and carbon flux through central metabolic pathways in Pseudomonas aeruginosa PA14. Journal of Bacteriology 2007;189: 6372-81.

263. Qadri SM, Donkor DA, Bhakta V et al. Phosphatidylserine externalization and procoagulant activation of erythrocytes induced by Pseudomonas aeruginosa virulence factor pyocyanin. Journal of Cellular and Molecular Medicine 2016;20: $710-20$.

264. Qiao S, Luo Q, Zhao Y et al. Structural basis for lipopolysaccharide insertion in the bacterial outer membrane. Nature 2014;511: 108-11.

265. Qiu D, Eisinger VM, Head NE et al. ClpXP proteases positively regulate alginate overexpression and mucoid conversion in Pseudomonas aeruginosa. Microbiology 2008a;154: 2119-30. 
266. Qiu D, Eisinger VM, Rowen DW et al. Regulated proteolysis controls mucoid conversion in Pseudomonas aeruginosa. Proceedings of the National Academy of Sciences of the United States of America 2007;104: 8107-12.

267. Qiu DR, Esinger VM, Head NE et al. ClpXP proteases positively regulate alginate overexpression and mucoid conversion in Pseudomonas aeruginosa. Microbiology 2008b;154: 2119-30.

268. Quinton PM. Chloride impermeability in cystic fibrosis. Nature 1983;301: 421-2.

269. Rada B, Leto TL. Pyocyanin effects on respiratory epithelium: relevance in Pseudomonas aeruginosa airway infections. Trends in Microbiology 2013;21: 7381.

270. Raetz CR, Whitfield C. Lipopolysaccharide endotoxins. Annual Review Biochemistry 2002;71: 635-700.

271. Rahme LG, Stevens EJ, Wolfort SF et al. Common virulence factors for bacterial pathogenicity in plants and animals. Science 1995;268: 1899-902.

272. Ramsey DM, Wozniak DJ. Understanding the control of Pseudomonas aeruginosa alginate synthesis and the prospects for management of chronic infections in cystic fibrosis. Molecular Microbiology 2005;56: 309-22.

273. Ran H, Hassett DJ, Lau GW. Human targets of Pseudomonas aeruginosa pyocyanin. Proceedings of the National Academy of Sciences of the United States of America 2003;100: 14315-20.

274. Ranjan KP, Ranjan N, Bansal SK et al. Prevalence of Pseudomonas aeruginosa in post-operative wound infection in a referral hospital in Haryana, India. Journal of Lab Physicians 2010;2: 74-7.

275. Raschle T, Amrhein N, Fitzpatrick TB. On the two components of pyridoxal 5 'phosphate synthase from Bacillus subtilis. Journal of Biological Chemistry 2005;280: 32291-300.

276. Recinos DA, Sekedat MD, Hernandez A et al. Redundant phenazine operons in Pseudomonas aeruginosa exhibit environment-dependent expression and 
differential roles in pathogenicity. Proceedings of the National Academy of Sciences of the United States of America 2012;109: 19420-5.

277. Regni C, Naught L, Tipton PA et al. Structural basis of diverse substrate recognition by the enzyme PMM/PGM from Pseudomonas aeruginosa. Structure 2004;12: 55-63.

278. Reiling SA, Jansen JA, Henley BJ et al. Prc protease promotes mucoidy in mucA mutants of Pseudomonas aeruginosa. Microbiology 2005;151: 2251-61.

279. Rello J, Jubert P, Valles J et al. Evaluation of outcome for intubated patients with pneumonia due to Pseudomonas aeruginosa. Clinical Infectious Diseases 1996;23: 973-8.

280. Remminghorst U, Hay ID, Rehm BHA. Molecular characterization of Alg8, a putative glycosyltransferase, involved in alginate polymerisation. Journal of Biotechnology 2009;140: 176-83.

281. Remminghorst U, Rehm BH. In vitro alginate polymerization and the functional role of Alg8 in alginate production by Pseudomonas aeruginosa. Applied and Environmental Microbiology 2006;72: 298-305.

282. Rice LB. Progress and challenges in implementing the research on ESKAPE pathogens. Infection Control and Hospital Epidemiology 2010;31: S7-S10.

283. Rietsch A, Vallet-Gely I, Dove SL et al. ExsE, a secreted regulator of type III secretion genes in Pseudomonas aeruginosa. Proceedings of the National Academy of Sciences of the United States of America 2005;102: 8006-11.

284. Righi E, Peri AM, Harris PNA et al. Global prevalence of carbapenem resistance in neutropenic patients and association with mortality and carbapenem use: systematic review and meta-analysis. Journal of Antimicrobial Chemotherapy 2017;72: 668-77.

285. Riordan JR, Rommens JM, Kerem BS et al. Identification of the cystic fibrosis gene - cloning and characterization of complementary-DNA. Science 1989;245: 1066-72. 
286. Robles-Price A, Wong TY, Sletta $\mathbf{H}$ et al. AlgX is a periplasmic protein required for alginate biosynthesis in Pseudomonas aeruginosa. Journal of Bacteriology 2004;186: 7369-77.

287. Romling U, Fiedler B, Bosshammer $\mathbf{J}$ et al. Epidemiology of chronic Pseudomonas aeruginosa infections in cystic fibrosis. Journal of Infectious Diseases 1994;170: 1616-21.

288. Rommens JM, Iannuzzi MC, Kerem B et al. Identification of the cystic fibrosis gene: chromosome walking and jumping. Science 1989;245: 1059-65.

289. Rosenfeld Y, Papo N, Shai Y. Endotoxin (lipopolysaccharide) neutralization by innate immunity host-defense peptides. Peptide properties and plausible modes of action. Journal Biology Chemistry 2006;281: 1636-43.

290. Rouviere PE, Gross CA. SurA, a periplasmic protein with peptidyl-prolyl isomerase activity, participates in the assembly of outer membrane porins. Genes \& Development 1996;10: 3170-82.

291. Rowen DW, Deretic V. Membrane-to-cytosol redistribution of ECF sigma factor $\mathrm{Alg} \mathrm{U}$ and conversion to mucoidy in Pseudomonas aeruginosa isolates from cystic fibrosis patients. Molecular Microbiology 2000;36: 314-27.

292. Roychoudhury S, May TB, Gill JF et al. Purification and characterization of guanosine diphospho-D-mannose dehydrogenase - a Key enzyme in the biosynthesis of alginate by Pseudomonas aeruginosa. Journal of Biological Chemistry 1989;264: 9380-5.

293. Rubino R, Bezzerri V, Favia M et al. Pseudomonas aeruginosa reduces the expression of CFTR via post-translational modification of NHERF1. Pflugers Archiv-European Journal of Physiology 2014;466: 2269-78.

294. Ruiz N, Chng SS, Hiniker A et al. Nonconsecutive disulfide bond formation in an essential integral outer membrane protein. Proceedings of the National Academy of Sciences of the United States of America 2010;107: 12245-50.

295. Ruiz N, Gronenberg LS, Kahne D et al. Identification of two inner-membrane proteins required for the transport of lipopolysaccharide to the outer membrane of 
Escherichia coli. Proceedings of the National Academy of Sciences of the United States of America 2008;105: 5537-42.

296. Sabo DL, Fischer EH. Chemical properties of Escherichia coli lysine decarboxylase including a segment of Its pyridoxal 5'-phosphate binding-site. Biochemistry 1974;13: 670-6.

297. Sambrook J, Fritsch EF, Maniatis T. Molecular cloning: a laboratory manual: 2 Edition. Cold Spring Harbor, NY: Cold Spring Harbor Laboratory, 1989.

298. Sampson BA, Misra R, Benson SA. Identification and characterization of a new gene of Escherichia coli K-12 involved in outer membrane permeability. Genetics 1989;122: 491-501.

299. Sautter R, Ramos D, Schneper L et al. A complex multilevel attack on Pseudomonas aeruginosa AlgT/U expression and AlgT/U activity results in the loss of alginate production. Gene 2012;498: 242-53.

300. Schiller NL, Monday SR, Boyd CM et al. Characterization of the Pseudomonas aeruginosa alginate lyase gene (Algl) - cloning, sequencing, and expression in Escherichia coli. Journal of Bacteriology 1993;175: 4780-9.

301. Schobel S, Zellmeier S, Schumann W et al. The Bacillus subtilis sigmaW antisigma factor RsiW is degraded by intramembrane proteolysis through YluC. Molecular Microbiology 2004;52: 1091-105.

302. Schurks N, Wingender J, Flemming HC et al. Monomer composition and sequence of alginates from Pseudomonas aeruginosa. International Journal of Biological Macromolecules 2002;30: 105-11.

303. Schurr MJ, Martin DW, Mudd MH et al. Gene-cluster controlling conversion to alginate overproducing phenotype in Pseudomonas aeruginosa - functionalanalysis in a heterologous host and role in the instability of mucoidy. Journal of Bacteriology 1994;176: 3375-82.

304. Schurr MJ, Yu H, Martinez-Salazar JM et al. Control of AlgU, a member of the sigma E-like family of stress sigma factors, by the negative regulators MucA and MucB and Pseudomonas aeruginosa conversion to mucoidy in cystic fibrosis. Journal of Bacteriology 1996;178: 4997-5004. 
305. Schwalm J, Mahoney TF, Soltes GR et al. Role for Skp in LptD assembly in Escherichia coli. Journal of Bacteriology 2013;195: 3734-42.

306. Schwarzmann S, Boring JR. Antiphagocytic effect of slime from a mucoid strain of Pseudomonas aeruginosa. Infection and Immunity 1971;3: 762-7.

307. Scoffone VC, Chiarelli LR, Trespidi G et al. Burkholderia cenocepacia infections in Cystic Fibrosis patients: drug resistance and therapeutic approaches. Frontiers in Microbiology 2017;8: 1592.

308. Scott FW, Pitt TL. Identification and characterization of transmissible Pseudomonas aeruginosa strains in cystic fibrosis patients in England and Wales. Journal of Medical Microbiology 2004;53: 609-15.

309. Shanbhag R, Shi G, Rujiviphat $\mathbf{J}$ et al. The emerging role of proteolysis in mitochondrial quality control and the etiology of parkinson's disease. Parkinsons Disease 2012, DOI 10.1155/2012/382175.

310. Shanks RM, Caiazza NC, Hinsa SM et al. Saccharomyces cerevisiae based molecular tool kit for manipulation of genes from Gram-negative bacteria. Applied and Environmental Microbiology 2006;72: 5027-36.

311. Shanks RM, Kadouri DE, MacEachran DP et al. New yeast recombineering tools for bacteria. Plasmid 2009;62: 88-97.

312. Sheppard DN, Rich DP, Ostedgaard LS et al. Mutations in CFTR associated with mild-disease-form Cl- channels with altered pore properties. Nature 1993;362: 1604.

313. Shinabarger D, Berry A, May TB et al. Purification and characterization of phosphomannose isomerase-guanosine diphospho-D-mannose pyrophosphorylase - a bifunctional enzyme in the alginate biosynthetic pathway of Pseudomonas aeruginosa. Journal of Biological Chemistry 1991;266: 2080-8.

314. Shinabarger D, May TB, Boyd A et al. Nucleotide sequence and expression of the Pseudomonas aeruginosa alg $F$ gene controlling acetylation of alginate. Molecular Microbiology 1993;9: 1027-35. 
315. Shortridge VD, Pato ML, Vasil AI et al. Physical mapping of virulence associated genes in Pseudomonas aeruginosa by transverse alternating-field electrophoresis. Infection and Immunity 1991;59: 3596-603.

316. Silva Filho LV, Castanos C, Ruiz HH. Cystic fibrosis in latin america-improving the awareness. Journal of Cystic Fibrosis 2016;15: 791-3.

317. Silvis MR, Picciano JA, Bertrand $\mathbf{C}$ et al. A mutation in the cystic fibrosis transmembrane conductance regulator generates a novel internalization sequence and enhances endocytic rates. Journal of Biological Chemistry 2003;278: 1155460.

318. Simpson JA, Smith SE, Dean RT. Scavenging by alginate of free radicals released by macrophages. Free Radical Biology and Medicine 1989;6: 347-53.

319. Skjakbraek G, Grasdalen H, Larsen B. Monomer sequence and acetylation pattern in some bacterial alginates. Carbohydrate Research 1986;154: 239-50.

320. Skjakbraek G, Paoletti S, Gianferrara T. Selective acetylation of mannuronic acid residues in calcium alginate gels. Carbohydrate Research 1989;185: 119-29.

321. Sklar JG, Makinoshima H, Schneider JS et al. M. tuberculosis intramembrane protease Rip1 controls transcription through three anti-sigma factor substrates. Molecular Microbiology 2010;77: 605-17.

322. Smith EE, Buckley DG, Wu $\mathbf{Z}$ et al. Genetic adaptation by Pseudomonas aeruginosa to the airways of cystic fibrosis patients. Proceedings of the National Academy of Sciences of the United States of America 2006;103: 8487-92.

323. Smith PK, Krohn RI, Hermanson GT et al. Measurement of protein using bicinchoninic acid. Analytical Biochemistry 1985;150: 76-85.

324. Snook CF, Tipton PA, Beamer LJ. Crystal structure of GDP-mannose dehydrogenase: A key enzyme of alginate biosynthesis in Pseudomonas aeruginosa. Biochemistry 2003;42: 4658-68. 
325. Song $\mathbf{Z}, \mathbf{W u} \mathbf{H}$, Ciofu $\mathbf{O}$ et al. Pseudomonas aeruginosa alginate is refractory to Th1 immune response and impedes host immune clearance in a mouse model of acute lung infection. Journal of Medical Microbiology 2003;52: 731-40.

326. Speert DP, Farmer SW, Campbell ME et al. Conversion of Pseudomonas aeruginosa to the phenotype characteristic of strains from patients with cystic fibrosis. Journal of Clinical Microbiology 1990;28: 188-94.

327. Sperandeo P, Deho G, Polissi A. The lipopolysaccharide transport system of Gram-negative bacteria. Biochimica Et Biophysica Acta-Molecular and Cell Biology of Lipids 2009;1791: 594-602.

328. Srinivas N, Jetter P, Ueberbacher BJ et al. Peptidomimetic antibiotics target outer-membrane biogenesis in Pseudomonas aeruginosa. Science 2010;327: 10103.

329. Stapleton F, Edwards K, Keay $\mathbf{L}$ et al. Risk factors for moderate and severe microbial keratitis in daily wear contact lens users. Ophthalmology 2012;119: 1516-21.

330. Stapper AP, Narasimhan G, Ohman DE et al. Alginate production affects Pseudomonas aeruginosa biofilm development and architecture but is not essential for biofilm formation. Journal of Medical Microbiology 2004;53: 679-90.

331. Staskawicz B, Dahlbeck D, Keen $\mathbf{N}$ et al. Molecular characterization of cloned avirulence genes from race-0 and race-1 of Pseudomonas syringae Pv glycinea. Journal of Bacteriology 1987;169: 5789-94.

332. Stewart C, Pepper MS. Cystic fibrosis on the African continent. Genetics in Medicine 2016;18: 653-62.

333. Stoltz DA, Meyerholz DK, Welsh MJ. Origins of cystic fibrosis lung disease. New England Journal of Medicine 2015;372: 1574-5.

334. Strauch KL, Beckwith J. An Escherichia coli mutation preventing degradation of abnormal periplasmic proteins. Proceedings of the National Academy of Sciences of the United States of America 1988;85: 1576-80. 
335. Strauch KL, Johnson K, Beckwith J. Characterization of $\operatorname{deg} P$, a gene required for proteolysis in the cell envelope and essential for growth of Escherichia coli at high temperature. Journal of Bacteriology 1989a;171: 2689-96.

336. Strauch KL, Johnson K, Beckwith J. Characterization of $\operatorname{deg} P$, a gene required for proteolysis in the cell-envelope and essential for growth of Escherichia coli at high temperature. Journal of Bacteriology 1989b;171: 2689-96.

337. Tamir H, Srinivasan PR. Studies of the mechanism of anthranilate synthase. Effect of hydroxylamine and N-methylhydroxylamine. Journal of Biological Chemistry 1971;246: 3024-9.

338. Tang X, Pezzulo AA, Hoegger MJ et al. Reduced airway surface PH impairs bacterial killing in the porcine cystic fibrosis lung. Pediatric Pulmonology 2012;47: 227-.

339. Tatnell PJ, Russell NJ, Gacesa P. A metabolic study of the activity of GDPmannose dehydrogenase and concentrations of activated intermediates of alginate biosynthesis in Pseudomonas aeruginosa. Journal of General Microbiology 1993;139: 119-27.

340. Terry JM, Pina SE, Mattingly SJ. Environmental conditions which influence mucoid conversion in Pseudomonas aeruginosa PAO1. Infection and Immunity 1991;59: 471-7.

341. Thanassi DG, Hultgren SJ. Multiple pathways allow protein secretion across the bacterial outer membrane. Current Opinion in Cell Biology 2000;12: 420-30.

342. Tielen P, Strathmann M, Jaeger KE $\boldsymbol{e t}$ al. Alginate acetylation influences initial surface colonization by mucoid Pseudomonas aeruginosa. Microbiological Research 2005;160: 165-76.

343. Tormo A, Almiron M, Kolter R. SurA, an Escherichia coli gene essential for survival in stationary phase. Journal of Bacteriology 1990;172: 4339-47.

344. Toussaint B, Delicattree I, Vignais PM. Pseudomonas aeruginosa contains an IHF-like protein that binds to the algD promoter. Biochemical and Biophysical Research Communications 1993;196: 416-21. 
345. Trejo-Hernandez A, Andrade-Dominguez A, Hernandez M et al. Interspecies competition triggers virulence and mutability in Candida albicans-Pseudomonas aeruginosa mixed biofilms. The Journal of Multidisciplinary Journal of Microbial Ecology 2014;8: 1974-88.

346. Trinh NTN, Bilodeau C, Maille E $\boldsymbol{e t}$ al. Deleterious impact of Pseudomonas aeruginosa on cystic fibrosis transmembrane conductance regulator function and rescue in airway epithelial cells. European Respiratory Journal 2015;45: 1590-602.

347. Tsui LC, Buchwald M, Barker D et al. Cystic fibrosis locus defined by a genetically linked polymorphic DNA marker. Science 1985;230: 1054-7.

348. van Ewijk BE, van der Zalm MM, Wolfs TF et al. Prevalence and impact of respiratory viral infections in young children with Cystic Fibrosis: prospective cohort study. Pediatrics 2008;122: 1171-6.

349. van Hartingsveldt J, Stouthamer AH. Mapping and characterization of mutants of Pseudomonas aeruginosa affected in nitrate respiration in aerobic or anaerobic growth. Journal of General Microbiology 1973;74: 97-106.

350. Vecchietti D, Di Silvestre D, Miriani M et al. Analysis of Pseudomonas aeruginosa cell envelope proteome by capture of surface-exposed proteins on activated magnetic nanoparticles. Plos One 2012;7.

351. Veit G, Avramescu RG, Chiang AN et al. From CFTR biology toward combinatorial pharmacotherapy: expanded classification of cystic fibrosis mutations. Molecular Biology of the Cell 2016;27: 424-33.

352. Vertommen D, Ruiz N, Leverrier $\mathbf{P}$ et al. Characterization of the role of the Escherichia coli periplasmic chaperone SurA using differential proteomics. Proteomics 2009;9: 2432-43.

353. Vrolijk MF, Opperhuizen A, Jansen $\mathbf{E}$ et al. The vitamin $\mathrm{B}_{6}$ paradox: Supplementation with high concentrations of pyridoxine leads to decreased vitamin B6 function. Toxicology In Vitro 2017;44: 206-12.

354. Wainwright BJ, Scambler PJ, Schmidtke $\mathbf{J}$ et al. Localization of cystic fibrosis locus to human chromosome 7cen-q22. Nature 1985;318: 384-5. 
355. Waller PR, Sauer RT. Characterization of $\operatorname{deg} Q$ and $\operatorname{deg} S$, Escherichia coli genes encoding homologs of the DegP protease. Journal of Bacteriology 1996;178: 114653.

356. Walsh NP, Alba BM, Bose B et al. OMP peptide signals initiate the envelopestress response by activating DegS protease via relief of inhibition mediated by its PDZ domain. Cell 2003;113: 61-71.

357. Wang QP, Kaguni JM. A novel sigma factor is involved in expression of the $r p o H$ gene of Escherichia coli. Journal of Bacteriology 1989;171: 4248-53.

358. Wang XB, Dockery DW, Wypij D et al. Pulmonary-Function between 6 and 18 Years of Age. Pediatric Pulmonology 1993;15: 75-88.

359. Warren AE, Boulianne-Larsen CM, Chandler CB et al. Genotypic and phenotypic variation in Pseudomonas aeruginosa reveals signatures of secondary infection and mutator activity in certain cystic fibrosis patients with chronic lung infections. Infection and Immunity 2011;79: 4802-18.

360. Webster D, Bernheim F. Oxidation of amino acids by Bacillus pyocyaneus (Pseudomonas aeruginosa). Journal of Biological Chemistry 1936;114.

361. Werneburg M, Zerbe K, Juhas M et al. Inhibition of lipopolysaccharide transport to the outer membrane in Pseudomonas aeruginosa by peptidomimetic antibiotics. Chembiochem 2012;13: 1767-75.

362. Whitchurch CB, Alm RA, Mattick JS. The alginate regulator AlgR and an associated sensor FimS are required for twitching motility in Pseudomonas aeruginosa. Proceedings of the National Academy of Sciences of the United States of America 1996;93: 9839-43.

363. Wilken C, Kitzing K, Kurzbauer R et al. Crystal structure of the DegS stress sensor: How a PDZ domain recognizes misfolded protein and activates a protease. Cell 2004;117: 483-94.

364. Willger SD, Grim SL, Dolben EL et al. Characterization and quantification of the fungal microbiome in serial samples from individuals with cystic fibrosis. Microbiome 2014;2: 40. 
365. Williams D, Fothergill JL, Evans B et al. Transmission and lineage displacement drive rapid population genomic flux in cystic fibrosis airway infections of a Pseudomonas aeruginosa epidemic strain. Microbial Genomics 2018, DOI 10.1099/mgen.0.000167.

366. Winsor GL, Griffiths EJ, Lo $\mathbf{R}$ et al. Enhanced annotations and features for comparing thousands of Pseudomonas genomes in the Pseudomonas genome database. Nucleic Acids Research 2016;44: 646-53.

367. Winstanley C, O'Brien S, Brockhurst MA. Pseudomonas aeruginosa evolutionary adaptation and diversification in cystic fibrosis chronic lung infections. Trends in Microbiology 2016;24: 327-37.

368. Wloka M, Rehage H, Flemming HC et al. Rheological properties of viscoelastic biofilm extracellular polymeric substances and comparison to the behavior of calcium alginate gels. Colloid and Polymer Science 2004;282: 1067-76.

369. Wollmann P, Zeth K. The structure of RseB: A sensor in periplasmic stress response of E. coli. Journal of Molecular Biology 2007;372: 927-41.

370. Wolosker H, Blackshaw S, Snyder SH. Serine racemase: a glial enzyme synthesizing D-serine to regulate glutamate-N-methyl-D-aspartate neurotransmission. Proceeding of the National Academy of Sciences of the United States of America 1999;96: 13409-14.

371. Wood LF, Leech AJ, Ohman DE. Cell wall-inhibitory antibiotics activate the alginate biosynthesis operon in Pseudomonas aeruginosa: Roles of sigma (AlgT) and the AlgW and Prc proteases. Molecular Microbiology 2006;62: 412-26.

372. Wood LF, Ohman DE. Independent regulation of MucD, an HtrA-like protease in Pseudomonas aeruginosa, and the role of its proteolytic motif in alginate gene regulation. Journal of Bacteriology 2006;188: 3134-7.

373. Wood LF, Ohman DE. Use of cell wall stress to characterize sigma 22 (AlgT/U) activation by regulated proteolysis and its regulon in Pseudomonas aeruginosa. Molecular Microbiology 2009;72: 183-201.

374. Workentine ML, Sibley CD, Glezerson B et al. Phenotypic heterogeneity of Pseudomonas aeruginosa populations in a cystic fibrosis patient. Plos One 2013;8. 
375. Workman P, Heide K, Giuliano $\mathbf{N}$ et al. Genetic, biochemical, and molecular characterization of the polypeptide transport-associated domain of Escherichia coli BamA. Journal of Bacteriology 2012;194: 3512-21.

376. Worlitzsch D, Tarran R, Ulrich M et al. Effects of reduced mucus oxygen concentration in airway Pseudomonas infections of cystic fibrosis patients. Journal of Clinical Investigation 2002;109: 317-25.

377. Wozniak DJ, Ohman DE. Pseudomonas aeruginosa AlgB, a two-component response regulator of the $\mathrm{NtrC}$ family, is required for $\operatorname{alg} D$ transcription. Journal of Bacteriology 1991;173: 1406-13.

378. Wozniak DJ, Ohman DE. Transcriptional analysis of the Pseudomonas aeruginosa genes $\mathrm{AlgR}, \mathrm{AlgB}$, and $\mathrm{AlgD}$ reveals a hierarchy of alginate geneexpression which is modulated by AlgT. Journal of Bacteriology 1994;176: 600714.

379. Wozniak DJ, Sprinkle AB, Baynham PJ. Control of Pseudomonas aeruginosa algZ expression by the alternative sigma factor AlgT. Journal of Bacteriology 2003;185: 7297-300.

380. Wu T, McCandlish AC, Gronenberg LS et al. Identification of a protein complex that assembles lipopolysaccharide in the outer membrane of Escherichia coli. Proceedings of the National Academy of Sciences of the United States of America 2006;103: 11754-9.

381. Wurtzel O, Yoder-Himes DR, Han $\mathbf{K}$ et al. The single-nucleotide resolution transcriptome of Pseudomonas aeruginosa grown in body temperature. Plos Pathogens 2012;8.

382. Xie ZD, Hershberger CD, Shankar $\mathbf{S}$ et al. Sigma factor-anti-sigma factor interaction in alginate synthesis: inhibition of AlgT by MucA. Journal of Bacteriology 1996;178: 4990-6.

383. Xu BJ, Soukup RJ, Jones CJ et al. Pseudomonas aeruginosa AmrZ binds to four sites in the $\operatorname{alg} D$ promoter, inducing DNA-AmrZ complex formation and transcriptional activation. Journal of Bacteriology 2016;198: 2673-81. 
384. Xu X, Wang S, Hu YX et al. The periplasmic bacterial molecular chaperone SurA adapts its structure to bind peptides in different conformations to assert a sequence preference for aromatic residues. Journal of Molecular Biology 2007;373: 367-81.

385. Yamashiro Y, Shimizu T, Oguchi S $\boldsymbol{e t}$ al. The estimated incidence of cystic fibrosis in Japan. Journal of Pediatric Gastroenterology and Nutrition 1997;24: 544-7.

386. Yin Y, Withers TR, Wang $\mathbf{X}$ et al. Evidence for sigma factor competition in the regulation of alginate production by Pseudomonas aeruginosa. Plos One 2013;8: e72329.

387. Yoshida R, Nagata M, Nakayama $\mathbf{H}$ et al. The pathological significance of Notch1 in oral squamous cell carcinoma. Laboratory Investigation 2013;93: 106881.

388. Yu H, Hanes M, Chrisp CE et al. Microbial pathogenesis in cystic fibrosis: pulmonary clearance of mucoid Pseudomonas aeruginosa and inflammation in a mouse model of repeated respiratory challenge. Infection and Immunity 1998;66: 280-8.

389. Yu H, Mudd M, Boucher JC et al. Identification of the algZ gene upstream of the response regulator $a \lg R$ and its participation in control of alginate production in Pseudomonas aeruginosa. Journal of Bacteriology 1997;179: 187-93.

390. Yu H, Schurr MJ, Deretic V. Functional equivalence of Escherichia coli sigma E and Pseudomonas aeruginosa AlgU: E. coli rpoE restores mucoidy and reduces sensitivity to reactive oxygen intermediates in alg $U$ mutants of $P$. aeruginosa. Journal of Bacteriology 1995;177: 3259-68.

391. Yu JM, Chen ZH, Ni Y et al. CFTR mutations in men with congenital bilateral absence of the vas deferens (CBAVD): a systemic review and meta-analysis. Human Reproduction 2012;27: 25-35.

392. Zellmeier S, Schumann W, Wiegert T. Involvement of Clp protease activity in modulating the Bacillus subtilis sigmaW stress response. Molecular Microbiology 2006;61: 1569-82. 
393. Zha ZZ, Li CC, Li WY et al. LptD is a promising vaccine antigen and potential immunotherapeutic target for protection against Vibrio species infection. Scientific Reports 2016;6.

394. Zielenski J, Tsui LC. Cystic fibrosis: Genotypic and phenotypic variations. Annual Review of Genetics 1995;29: 777-807.

395. Zielinski NA, Chakrabarty AM, Berry A. Characterization and regulation of the Pseudomonas aeruginosa algC gene encoding phosphomannomutase. Journal of Biological Chemistry 1991;266: 9754-63.

396. Zielke RA, Wierzbicki IH, Baarda BI et al. Proteomics-driven antigen discovery for development of vaccines against gonorrhea. Molecular \& Cellular Proteomics 2016;15: 2338-55.

397. Zierdt CH, Schmidt PJ. Dissociation in Pseudomonas aeruginosa. Journal of Bacteriology 1964;87: 1003-10. 
VITA

\section{SUNDAR PANDEY}

2003-2007 Bachelor's in technology, Biotechnology

Kathmandu University, Dhulikhel, Kathmandu, Nepal

2014-2018 Doctoral Candidate

Florida International University, Miami, FL

$2010 \quad$ FIU- Presidential Fellowship

2016 NIH- Minority Biomedical research award [\$3,000]

\section{PUBLICATIONS AND PRESENTATIONS}

Sundar Pandey, Camila Delgado, Laura Florez, Hansi Kumari, and Kalai Mathee (2018). Outer membrane protein (PA0595) plays a role in the regulation of alginate synthesis in P. aeruginosa. Journal of Medical Microbiology.DOI:10.1099/jmm.0.000752

Sundar Pandey, Kyle L. Martins, and Kalai Mathee (2016). Posttranslational regulation of anti-sigma factor of RpoE: a comparison between the E. coli and P. aeruginosa systems. Wiley Blackwell Publications.

DOI: $10.1002 / 9781119004813 . \operatorname{ch} 32$

B. Colbert, H. Kumari, A. Pinon, L. Frey, S. Pandey, and K. Mathee (2018). Alginateregulating genes are identified in the clinical cystic fibrosis isolate of $P$. aeruginosa PA2192 bioRxiv 319004; doi: https://doi.org/10.1101/319004

Cold Spring harbor laboratory meeting, September 8-12, 2015. Sundar Pandey, Camila Delgado, Laura Florez, Hansi Kumari, and Kalai Mathee. Outer membrane protein (PA0595) plays a role in the regulation of alginate synthesis in P. aeruginosa

Graduate student appreciation week (GSAW), 04, 2015. Sundar Pandey, Camila Delgado, Laura Florez, Hansi Kumari, and Kalai Mathee. Outer membrane protein (PA0595) plays a role in the regulation of alginate synthesis in $P$. aeruginosa

ASM Microbiology, June 16-20, 2016. Sundar Pandey, Camila Delgado, Laura Florez, Hansi Kumari, and Kalai Mathee. Outer membrane protein (PA0595) plays a role in the regulation of alginate synthesis in $P$. aeruginosa 
$19^{\text {th }}$ annual biology research symposium (BSI), 02, 2017. Sundar Pandey, Camila Delgado, Laura Florez, Hansi Kumari, and Kalai Mathee. Outer membrane protein (PA0595) plays a role in the regulation of alginate synthesis in $P$. aeruginosa

$103^{\text {rd }}$ annual conference, ASM SEB, 11/ 2017. ASM Microbiology, June 16-20, 2016. Sundar Pandey, Camila Delgado, Laura Florez, Hansi Kumari, and Kalai Mathee. Outer membrane protein (PA0595) plays a role in the regulation of alginate synthesis in $P$. aeruginosa 